\title{
WestVirginiaUniversity
}

THE RESEARCH REPOSITORY @ WVU

Graduate Theses, Dissertations, and Problem Reports

2000

\section{Coupled nonlinear dynamical systems}

\author{
Hongyan Sun \\ West Virginia University
}

Follow this and additional works at: https://researchrepository.wvu.edu/etd

\section{Recommended Citation}

Sun, Hongyan, "Coupled nonlinear dynamical systems" (2000). Graduate Theses, Dissertations, and Problem Reports. 1215.

https://researchrepository.wvu.edu/etd/1215

This Dissertation is protected by copyright and/or related rights. It has been brought to you by the The Research Repository @ WVU with permission from the rights-holder(s). You are free to use this Dissertation in any way that is permitted by the copyright and related rights legislation that applies to your use. For other uses you must obtain permission from the rights-holder(s) directly, unless additional rights are indicated by a Creative Commons license in the record and/ or on the work itself. This Dissertation has been accepted for inclusion in WVU Graduate Theses, Dissertations, and Problem Reports collection by an authorized administrator of The Research Repository @ WVU.

For more information, please contact researchrepository@mail.wvu.edu. 


\title{
Coupled Nonlinear Dynamical Systems
}

\author{
Hongyan Sun
}

\author{
Dissertation submitted to the \\ Eberly College of Arts and Sciences \\ at West Virginia University \\ in Partial Fulfillment of the Requirements for \\ the Degree of
}

Doctor of Philosophy in Physics

Kenneth Showalter, Ph.D., Chair Larry Halliburton, Ph.D., Co-Chair Arthur Weldon, Ph.D. Mark Koepke, Ph.D. Martin Ferer, Ph.D.

Morgantown, West Virginia 2000 


\section{ABSTRACT \\ Coupled Nonlinear Dynamical Systems}

Hongyan Sun

In this dissertation, we study coupled nonlinear dynamical systems that exhibit new types of complex behavior. We numerically and analytically examine a variety of dynamical models, ranging from systems of ordinary differential equations (ODE) with novel elements of feedback to systems of partial differential equations (PDE) that model chemical pattern formation. Chaos, dynamical uncertainty, synchronization, and spatiotemporal pattern formation constitute the primary topics of the dissertation. Following the introduction in Chapter 1, we study chaos and dynamical uncertainty in Chapter 2 with coupled Lorenz systems and demonstrate the existence of extreme complexity in high-dimensional ODE systems. In Chapter 3, we demonstrate that chaos synchronization can be achieved by mutual and multiplicative coupling of dynamical systems. Chapter 4 and 5 focus on pattern formation in reaction-diffusion systems, and we investigate segregation and integration behavior of populations in competitive and cooperative environments, respectively. 


\section{ACKNOWLEDGEMENTS}

Foremost, I would like to thank my advisor, Dr. Kenneth Showalter, for not only guiding me through my research at WVU but also advising me on many other aspects of academic life.

I am also grateful to Dr. Larry Halliburton, Chairman of the Physics Department and a member of my examining committee, and Dr. Paul Jagodzinski, Chairman of the Chemistry Department, for their assistance in making the joint physics-chemistry research work smoothly.

Particular thanks are due to Drs. Stephen K. Scott, Renate Wackerbauer, Jichang Wang, and Michael Hilderbrand, with whom I enjoyed valuable discussion and cooperation. Many thanks to Drs. Eugene Mihaliuk, Boyd F. Edwards, Sandor Kardar, Mingzhou Ding, and Vilmos Gáspár, from whom I learned a great deal both inside and outside of my research field.

I also wish to thank my colleagues and friends, Florin Chirila, Jianxia Cui, Jianan Feng, and Henrik Skødt for their contributions in making our group friendly and intellectually stimulating.

Finally, special thanks to my wife, Yanmei Liu, for her endless support and patience. 


\section{TABLE OF CONTENTS}

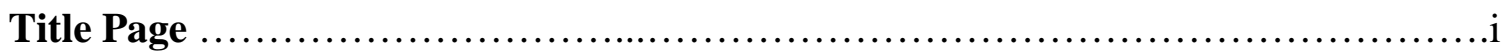

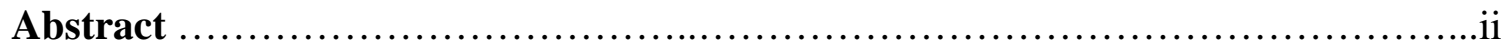

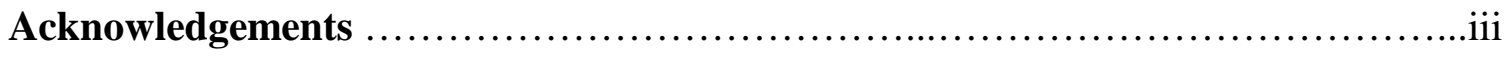

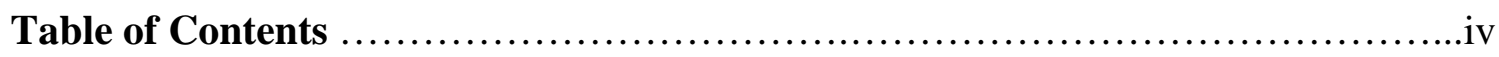

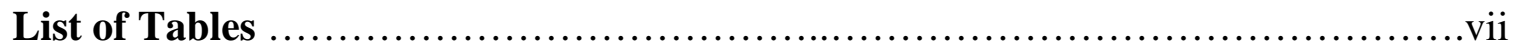

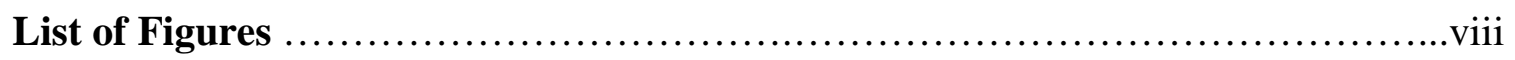

1. Introduction 1

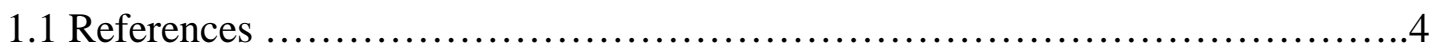

2. Uncertainty in high-dimensional chaotic dynamical systems 6

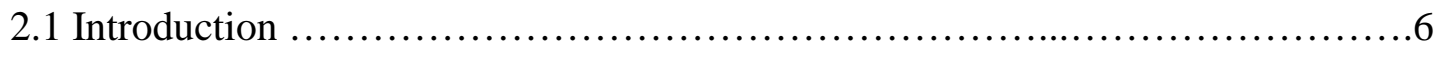

2.2 Chaotic behavior and riddled basins ....................................... 8

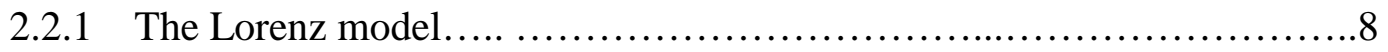

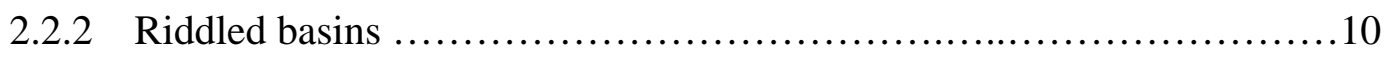

2.3 Uncertain destination dynamics ........................................13

2.3.1 General description ............................................13

2.3.2 Example: Two coupled Lorenz systems ..............................14

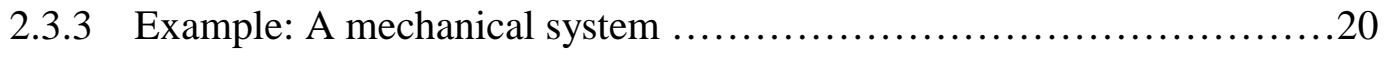

2.3.4 Example: Chemical kinetics ....................................23

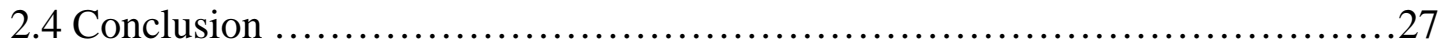

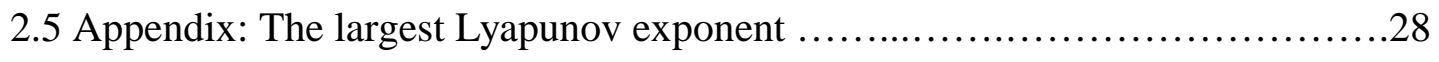

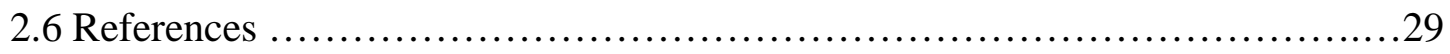




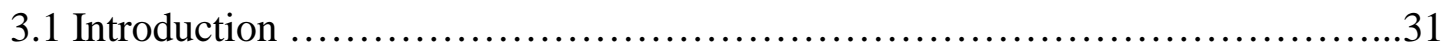

3.2 Classification of chaos synchronization ....................................... 33

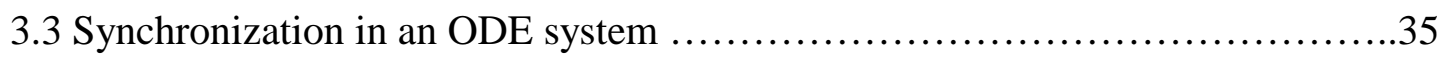

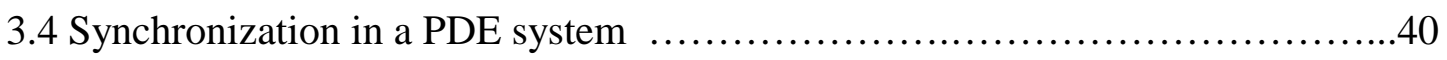

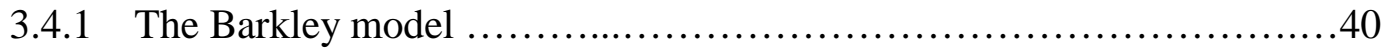

3.4.2 Pattern synchronization ..........................................44

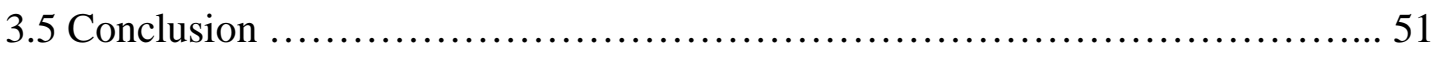

3.6 Appendix: Matlab program for the simulations ..............................52

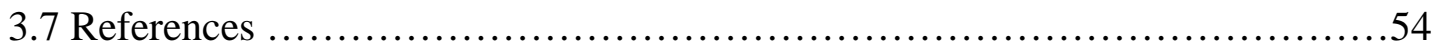

4. Self-segregation of competitive chaotic populations 57

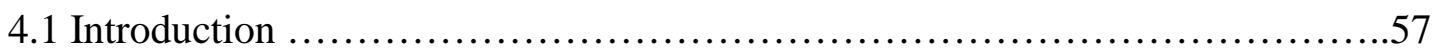

4.2 Illustration of Gray-Scott model without competition .........................59

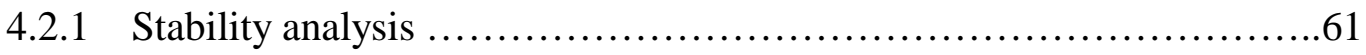

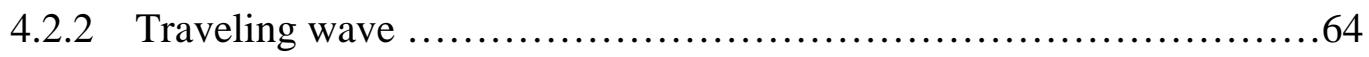

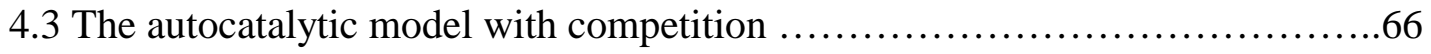

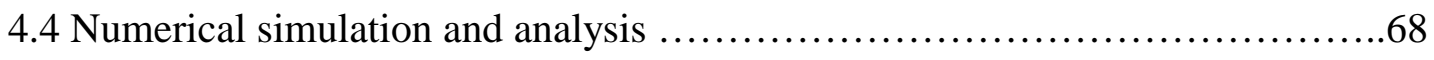

4.4.1 Self-segregation in one-dimensional medium ........................68

4.4.2 Pattern formation in 2D medium .................................. 76

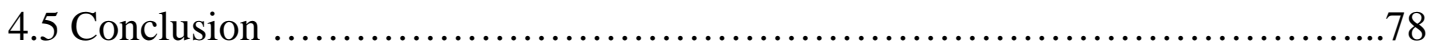

4.6 Appendix: The $\mathrm{C}$ program for the simulations …............................79

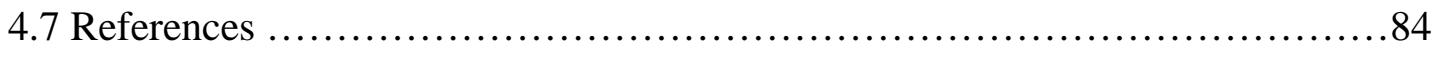




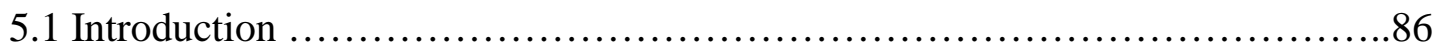

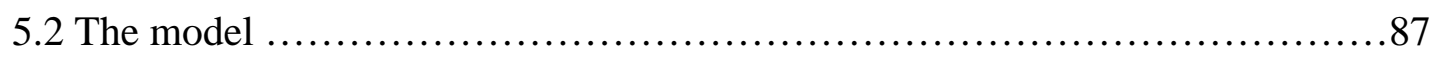

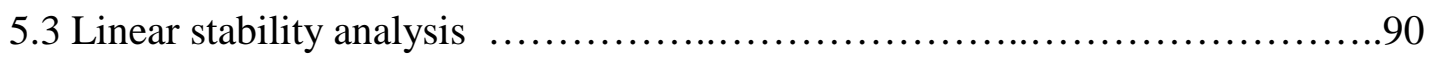

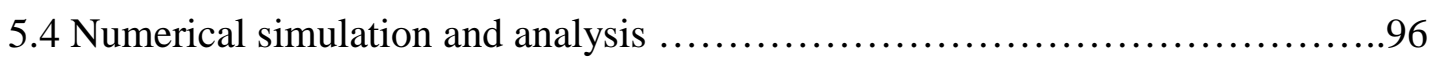

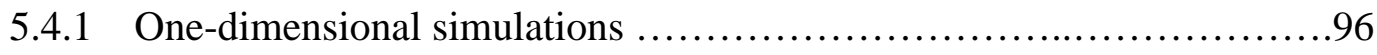

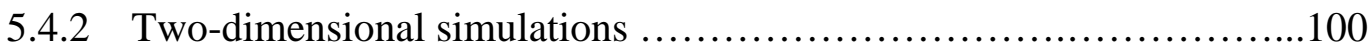

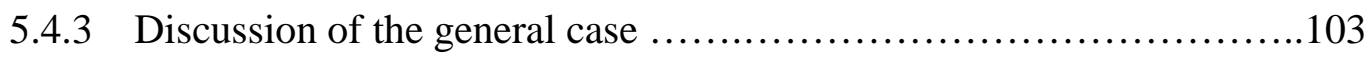

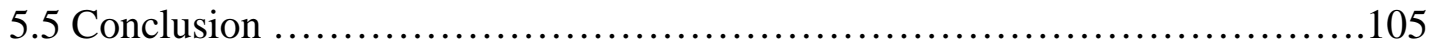

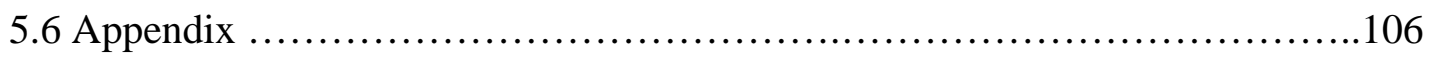

5.6.1 Exact solutions of the eigenvalues ...............................106

5.6.2 Matlab programs for the simulations .............................. 108

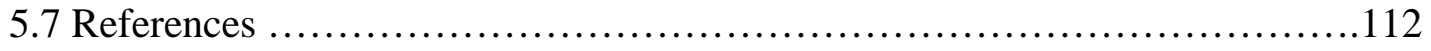




\section{LIST OF TABLES}

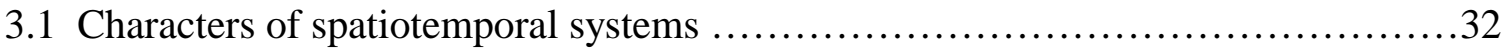




\section{LIST OF FIGURES}

2.1 A typical chaotic attractor in the Lorenz model .............................. 9

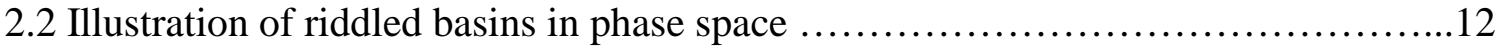

2.3 Time evolution of coupled Lorenz systems showing dynamical uncertainty ........15

2.4 (a) 2D plot of the largest Lyapunov exponents and (b) corresponding bifurcations ..16

2.5 Bifurcation sequence of destination dynamics ................................ 19

2.6 Time evolution of a mechanical system showing dynamical uncertainty ...........22

2.7 Bifurcation sequence of a chemical reaction model vs. initial conditions............25

2.8 The largest Lyapunov exponents vs. initial conditions in the reaction model .........26

3.1 Classification of chaos synchronization based on asymptotic states ................34

3.2 Classification of chaos synchronization based on coupling schemes ................34

3.3 "Shift synchronization" in the coupled Lorenz system ............................

3.4 "Expansion synchronization" in the coupled Lorenz system $\ldots \ldots \ldots \ldots \ldots \ldots \ldots \ldots \ldots . .38$

3.5 Illustration of multiple coupled systems for chaos synchronization ................39

3.6 Illustration of the nullclines in the Barkley model $\ldots \ldots \ldots \ldots \ldots \ldots \ldots \ldots \ldots \ldots \ldots \ldots .42$

3.7 A simulation of counterrotating spiral waves with the Barkley model .............43

3.8 Illustration of the nullclines in the coupled Barkley systems ....................46

3.9 An ODE simulation (local dynamics) shows the excitation of $w$ due to coupling ...48

3.10 2D simulation of the coupled Barkley model with regular initial conditions ... ....49

3.11 2D simulation of the coupled Barkley model with random initial conditions .........50

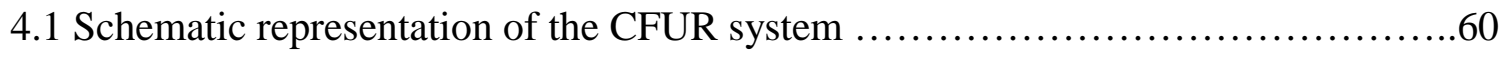

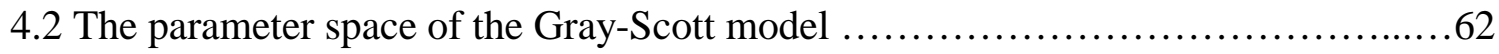


4.3 Typical instantaneous plots of the $\alpha$-profiles ...............................65

4.4 Spatiotemporal patterns of reactant and autocatalyst concentrations ...............69

4.5 Transient spatiotemporal dynamics of reactant and autocatalysts ...................70

4.6 Representative concentration profiles of the reactant and autocatalysts ............72

4.7 Characteristic phase portraits of local dynamics ...............................73

4.8 Long-term spatiotemporal dynamics of the reactant concentration ..................74

4.9 Spatiotemporal evolution of the reactant in a $2 \mathrm{D}$ simulation ........................77

5.1 The critical surface determining the existence of $S_{ \pm}{ }^{I}$ in the case of $v_{\mathrm{B}}=v_{\mathrm{C}} \ldots \ldots \ldots . .92$

5.2 Real part of the largest eigenvalue for the homogeneous steady states .............93

5.3 The existence and stability of $S_{-}{ }^{I}$ in the $2 \mathrm{D}$ parameter plane $v_{\mathrm{B}}$ vs. $v_{\mathrm{C}} \ldots \ldots \ldots \ldots \ldots 9$

$5.41 \mathrm{D}$ simulations of the integration model f....................................98

5.5 Measured average disappearing time of segregation w.r.t. parameter $v$ near $v_{\mathrm{c}} \ldots \ldots .99$

5.6 Spatiotemporal evolution of the resource in 2D simulations ......................101

5.7 Spatiotemporal evolution of the species in 2D simulations ......................102

5.8 A 1D demonstration of the case with symmetry broken $\left(v_{\mathrm{B}} \neq v_{\mathrm{C}}\right) \ldots \ldots \ldots \ldots \ldots \ldots . \ldots 104$ 


\section{Chapter 1}

\section{Introduction}

Over the past two decades there has been an enormous increase in the number of studies of nonlinear dynamics and complexity. Researchers have focused on exploring the essential conditions for nonlinear dynamical systems to exhibit complex dynamical behavior, for example, chaos. These studies have primarily utilized low-dimensional dynamical systems. ${ }^{1-3}$ Coupled nonlinear dynamical systems have also attracted attention in many scientific disciplines ${ }^{4-6}$ because of their potential applications. ${ }^{7-9}$ Systems that are structurally coupled typically show dynamical behavior different from that of isolated low-dimensional systems. For example, uncertainty in dynamics, ${ }^{10-12}$ chaos synchronization, ${ }^{13-16}$ and spatiotemporal $\operatorname{chaos}^{17}$ all arise from coupled high-dimensional dynamical systems.

In Chapter 2, we begin with investigations of dynamical complexity in ordinary differential equation (ODE) systems, namely uncertain destination dynamics. Exploring complex dynamical behavior related to riddled basins, ${ }^{11}$ which were recently discovered in high-dimensional dynamical systems, motivates this study. We present three specific examples to illustrate that some dynamical systems may have a foliation of attractors in phase space and the system may evolve to qualitatively different attractors, depending sensitively on initial conditions. ${ }^{18}$ The asymptotic dynamical behavior exhibited by the system can be studied through a reduction of the original high-dimensional dynamical system to its destination dynamics. Such a reduction introduces a constant in the 
equations describing the destination dynamics, but the value of the constant depends on the initial conditions of the original system.

Chaos synchronization has been the subject of many recent studies in nonlinear dynamics, and various schemes and techniques have been developed to achieve it. ${ }^{13}$ However, systems with mutual and multiplicative coupling schemes have not received as much attention. In Chapter 3, we describe our studies of chaos synchronization and spatiotemporal synchronization in mutually and multiplicatively coupled dynamical systems. ${ }^{19}$ We have used coupled Lorenz systems to study chaos synchronization and found two types of phase synchronization: The first type shows that, upon synchronization, the amplitude of a variable in one subsystem has a translational shift with respect to that of the corresponding variable in the other subsystem. The second type shows that the amplitude of a variable in one subsystem is proportional to that of the corresponding variable in the other subsystem. Moreover, the phase shift and proportionality depend on the initial conditions of the coupled system. For spatiotemporal synchronization, we have used a coupled Barkley model ${ }^{20}$ to demonstrate that spiral waves in excitable media can synchronize through mutual and multiplicative couplings.

Spatiotemporal systems have an infinite number of degrees of freedom, and it is generally more difficult to analyze their dynamics than ODE systems. In Chapter 4, we study the dynamical behavior of species competing for a common resource with a reaction-diffusion system based on cubic autocatalysis. ${ }^{21}$ We find that randomly seeded populations self-segregate to form a complex network of domains separated by distinct interfaces. In adjacent domains, different species are extinct while the other species exhibits chaotic oscillations. For chaotic populations in one-dimensional media, the interfaces exhibit irregular motions on long time scales, due to the uncorrelated chaotic oscillations on each side of the interfaces. In two-dimensional media, the interface motion is governed by curvature-induced drift.

The phenomenon of segregation is based on the competition between different species. However, species can also be cooperative while competing for a common 
resource. In Chapter 5, we add cooperative reaction steps into the model that is analyzed in the previous chapter, which was found to exhibit the phenomenon of segregation. ${ }^{22} \mathrm{We}$ find, depending on the relative intensity of the cooperative and the competitive processes, that the phenomenon of integration may occur in favor of segregation. Specifically, there is a transition from segregation to integration on increasing the importance of cooperation. At the critical values of the parameters, the system exhibits chaotic integration, while uniform integration is exhibited beyond the critical parameter values. 


\subsection{References}

1. J. Guckenheimer and P. Homes, Nonlinear Osillations, Dynamical Systems, and Bifurcations of Vector Fields (Springer-Verlag, New York, 1983).

2. J. C. Sprott, Phys. Rev. E 50, R647 (1995).

3. J. C. Sprott, Phys. Lett. A 228, 271 (1997).

4. M. Bulmer, Theoretical Evolutionary Ecology, (Sinauer Associates, Sunderland, Massachusetts, 1994).

5. H. Haken, Advanced Synergetics (Springer, Berlin, Heidelberg, 1983).

6. S. K. Scott, Chemical Chaos (Clarendon Press, Oxford, 1991).

7. M. P. Shaw, H. L. Grubin, E. Scholl, The Physics of Instabilities in Solid State Electron Devices (Academic, New York 1989)

8. K. M. Cuomo and Z. V. Oppenheim, Phys. Rev. Lett. 71, 65 (1993).

9. R. Roy, T. W. Murphy, T. D. Maiser, Z. Gills, and E. R. Hunt, Phys. Rev. Lett. 68, 1259 (1992).

10. J. C. Alexander, I. Kan, J. A. Yorke, and Z. You, Int. J. Bifurcation Chaos Appl. Sci. Eng. 2, 795 (1992).

11. J. C. Sommerer and E. Ott, Nature (London) 365, 136 (1993).

12. Y. C. Lai and R. L. Winslow, Phys. Rev. Lett. 72, 1640 (1994).

13. H. Fujisaka and T. Yamada, Prog. Theor. Phys. 69, 32 (1983).

14. A. S. Pikovsky, Z. Phys. B 55, 149 (1984).

15. V. S. Afraimovich, N. N. Verichev, and M. I. Rabinovich, Radiophys. Quantum Electron. 29, 795 (1986).

16. L. M. Pecora and T. L. Carroll, Phys. Rev. Lett. 64, 821 (1990).

17. M. C. Cross and P. C. Hohenberg, Rev. Mod. Phys. 65, 851 (1993).

18. H. Sun, S. K. Scott, and K. Showalter, Phys. Rev. E 60, 3876 (1999). 
19. H. Sun and K. Showalter, in preparation.

20. D. Barkley, Physica D 49, 61 (1991).

21. R. Wackerbauer, H. Sun, and K. Showalter, Phys. Rev. Lett 84, 5018 (2000).

22. H. Sun and K. Showalter, in preparation. 


\section{Chapter 2}

\section{Uncertainty in High-Dimensional Chaotic Dynamical Systems}

\subsection{Introduction}

Recent studies of high-dimensional dynamical systems have revealed many new modes of complex evolution, such as chaos synchronization, ${ }^{1-7}$ on-off intermittency, ${ }^{8-13}$ and fractal, riddled, and intermingled basins. ${ }^{14-20}$ Many of these studies have raised fundamental questions concerning the relationship between model systems and the real systems they attempt to describe. ${ }^{21-24}$ Here, the term "high-dimensional" refers to the topological dimension of phase space, which is larger than the minimum dimension of phase space that can contain a chaotic attractor. For example, in the theory of dynamical systems, a chaotic attractor in a discrete mapping system requires at least onedimensional phase space, while a continuous dynamical system requires at least three-

dimensional phase space. ${ }^{25-27}$ In this chapter, we study continuous dynamical systems with the dimension of phase space larger than three.

Low-dimensional dynamical systems can exhibit such asymptotic states as a fixed point, a limit cycle, a torus, or chaos. Chaos means extreme sensitivity of a dynamical system to initial conditions: Infinitesimally separated initial conditions result in an exponential divergence of the neighboring trajectories, although the dynamics is confined onto an invariant manifold. ${ }^{26}$ (An invariant manifold is a manifold such that any trajectory initialized in that manifold remains there for all time.) Most nonlinear dynamical systems are not solvable analytically, so computation is necessary to obtain a 
solution of the dynamical system. However, in the presence of chaos and inevitable round-off error, we cannot exactly predict the long-term behavior of a single trajectory, because of the exponential divergence of neighboring trajectories subject to small perturbations. That is, chaos essentially induces computational complexity. ${ }^{28}$

Statistical properties of a dynamical system, however, are characteristic quantities and, despite chaos, we may still have preserved ergodic measures that are independent of a particular trajectory or initial conditions. This is analogous to thermodynamics: We do not need to know the exact motion of the particles in an equilibrium in order to calculate the pressure, temperature, etc. of the equilibrium system based on the laws of statistical mechanics.

Another type of uncertainty in deterministic dynamical systems is shown by the phenomenon of riddled basins, ${ }^{14}$ which were discovered in the early 1990s, and demonstrated to occur only in a high-dimensional dynamical system. In the presence of riddled basins, infinitesimally separated initial conditions may lead to different asymptotic states of the dynamical system, which have different statistical properties. The existence of riddled basins was demonstrated to be not only a mathematical "symptom" in the theory of dynamics but also a reality in physical systems. In 1993, Sommerer and Ott ${ }^{15,16}$ provided a mechanical model that exhibits the phenomenon of riddled basins. Around the same period, several experiments were conducted by different groups of researchers that proved evidence for the existence of riddled basins. From the point of view of experimentalists, riddled basins impose a serious challenge to our belief in the "reproducibility" of experiments. This is also an example among many others showing that increasing the topological dimension of nonlinear dynamical systems does not merely induce a quantitative change of computational complexity.

Riddled basins occur in a dynamical system with a finite number of attractors. Although it is impossible to predict the asymptotic state of a randomly chosen initial condition inside the riddled basins, one may still have a probability measure to quantify the occurring frequency of each asymptotic state. ${ }^{22}$ In this chapter, we present examples 
that demonstrate a gross uncertainty resulting from initial conditions. ${ }^{29}$ In these examples, the number of asymptotic states is infinite; neighboring initial conditions may evolve to an infinite number of different destinations.

This chapter is organized as follows: In Section 2.2, we review related concepts for nonlinear dynamical systems by introducing the Lorenz model and the concept of riddled basins. In Section 2.3, we present specific dynamical systems and an analysis of the uncertain destination dynamics. In Section 2.4, we draw our conclusions.

\subsection{Chaotic Behavior and Riddled Basins}

\subsubsection{The Lorenz Model}

In 1963, Lorenz ${ }^{30,31}$ presented an analysis of a coupled set of three quadratic ordinary differential equations (ODEs) representing three modes of the Oberbeck-Boussinesq equations for fluid convection in a two-dimensional layer heated from below. While the phenomena displayed by the model have little quantitative similarity to actual phenomena occurring in convection, the model was extremely important in demonstrating the existence of chaos in coupled ODEs.

The dimensionless Lorenz model is given by:

$$
\begin{aligned}
& \dot{x}_{1}=\sigma\left(x_{2}-x_{1}\right), \\
& \dot{x}_{2}=r x_{1}-x_{2}-x_{1} y_{3}, \\
& \dot{x}_{3}=x_{1} x_{2}-b x_{3} .
\end{aligned}
$$

The three parameters $\sigma, r$, and $b$, which are all non-negative, correspond to the Prandtl number, the Rayleigh number, and an aspect ratio in the original sense of the fluid model. 


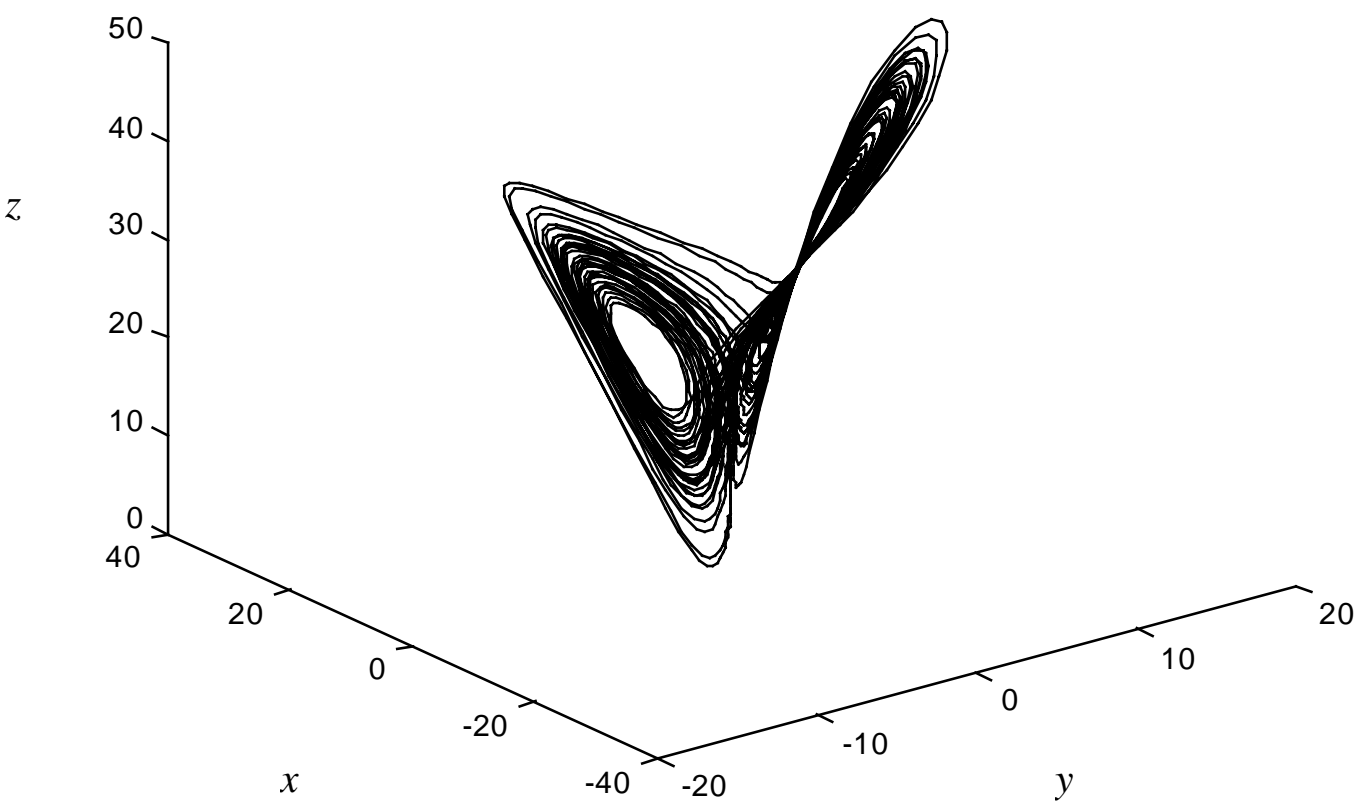

Figure 2.1. A typical chaotic attractor in the Lorenz model. $\sigma=10, b=$ $8 / 3$, and $r=28$. 
To study the chaotic phenomena and the bifurcation behavior exhibited by this model, we can fix the two parameters $\sigma$ and $b$, and let $r$ vary. The parametric values chosen by Lorenz and most other researchers are $\sigma=10, b=8 / 3$, but similar dynamical behavior can be observed for other parameter conditions. This model represents a dissipative dynamical system, according to the negative trace of the Jacobian, $-(\sigma+1+b)$, and thus it is possible that the system evolves to an attractor in phase space. (An attractor is a subset of the phase space of a dissipative dynamical system to which the system state asymptotically evolves). For $r<1$, the origin is a hyperbolic sink and is the only attractor in the system. At $r=1$, a pitchfork bifurcation of the steady state occurs such that for $r>$ 1 , the system exhibits two additional fixed points in phase space. When $r$ is further increased to a critical value $r_{\mathrm{H}}$, the two steady states lose stability and a Hopf bifurcation occurs, which results in the appearance of periodic oscillations. As $r$ is increased beyond the critical value, through a series of period-doubling bifurcations, the system can show chaotic behavior within some parameter ranges. Lorenz numerically demonstrated the chaotic behavior at $r=28$ with the other two parameter values chosen as $\sigma=10$ and $b=$ $8 / 3$ (Fig. 2.1). In fact, within a certain parameter range where chaos appears, there may be interspersed parameter intervals in which periodic behavior is exhibited. The periodic "windows" may be dense in the parameter space. The period-doubling bifurcation, chaos, and periodic windows can be found around $r=160$, where a tiny change of the parameter $r$ can shift the system from a periodic state to another periodic or chaotic state. These parameter values will be used for our discussion of uncertain destination dynamics in Section 2.3.

\subsubsection{Riddled Basins}

We introduce a mechanical model proposed by Sommerer and Ott $^{15}$ in 1993, which shows riddled basins: 


$$
\frac{d^{2}}{d t^{2}} r=-\gamma \frac{d}{d t} r-\nabla V(r)+\hat{x} p \sin \omega t
$$

with the scalar potential

$$
V(x, y)=\left(1-x^{2}\right)^{2}+(x-\bar{x}) y^{2},
$$

where $\gamma, p, \omega$ and $\bar{x}$ are parameters. This model describes a unit mass particle moving on a plane, and the motion is subject to friction, potential, and periodic driving forces. In terms of dynamics, the system has a five-dimensional phase space, which is spanned by $x, y, v_{x}, v_{y}$, and $\phi=\omega$. Owing to the symmetry $V(x, y) \equiv V(x,-y)$, the system has an invariant subspace at $y=v_{y}=0$ and, within the subspace, Eq. (2.2) is just the forced double-well Duffing equation $\left(\ddot{x}+\not \dot{x}-a x+b x^{3}=p \sin \omega t\right)$. With suitably chosen parameter values, the Duffing equation exhibits period-doubling and chaotic behavior. Sommerer and Ott focused on studying the dynamical behavior of Eq. (2.2) with the parameter values $\gamma=0.05, p=2.3, \omega=3.5$ and $\bar{x}=-1.9$. Under these conditions, the system has a chaotic attractor within the invariant plane and also a pair of periodic attractors symmetrically located outside this plane. Each of these attractors has its own basin of attraction, which consists of all the initial conditions attracted to it during the evolution of the system. Before the discovery of riddled basins, the basins of attractors in a dynamical system were found to separate regularly or at most with fractal boundaries. However, with the above chosen parameter values, the system exhibits a new phenomenon: The basins of those attractors are riddled by each other. If we draw a ball in phase space around an arbitrary initial condition that belongs to the attraction basin of one attractor, we can always find initial conditions within the ball, which belong to the basin of another attractor, no matter how small the ball is (Fig. 2.2). This means that a tiny perturbation to an initial condition that targets to one attractor may lead the system to the other attractor, where the two attractors have completely different dynamical properties. From the point of view of computation, such a perturbation may be round-off error, which is inevitable in computing. 


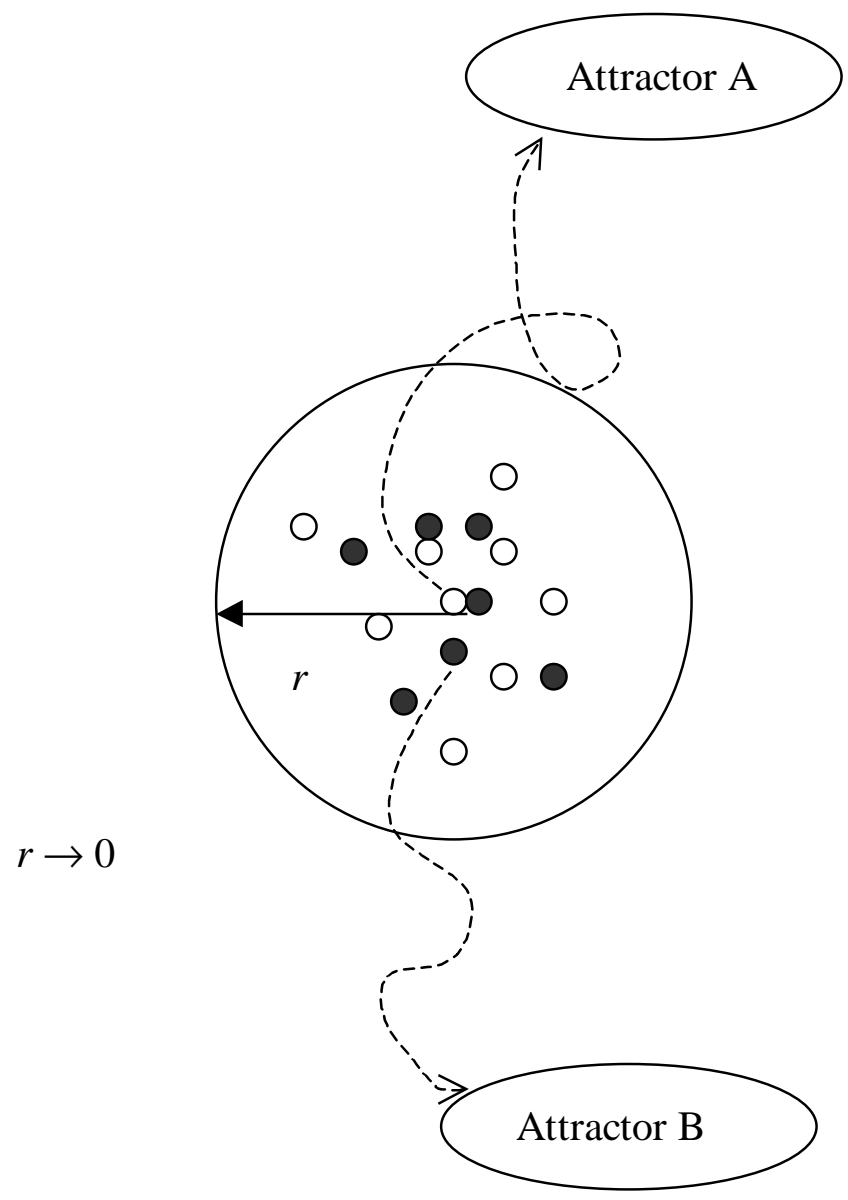

Figure 2.2. Illustration of riddled basins in phase space. Small circles and dots represent initial conditions, which attract to attractor A and B, respectively. $r$ can be arbitrarily small. Attractors can be fixed points, limit cycles, tori, or chaos. 


\subsection{Uncertain Destination Dynamics}

\subsubsection{General Description}

For many dynamical systems governed by evolution equations of the form

$$
\dot{x}_{i}=f_{i}\left(x_{1}, \ldots, x_{n}\right), i=1, \ldots, n
$$

the long-time evolution can be described by a reduced system,

$$
\dot{x}_{i}=g_{i}\left(x_{1}, \ldots, x_{m} ; c_{1}, \ldots c_{k}\right), i=1, \ldots, m, k \leq n-m
$$

along with a series of algebraic relations,

$$
0=h_{j}\left(x_{1}, \ldots, x_{n} ; c_{j}\right), j=1, \ldots, k
$$

where $c_{j}$ are constants. In general, these constants will depend on the initial conditions of the full system,

$$
c_{j}=c_{j}\left(x_{0 i}\right), i=1, \ldots, n
$$

We may refer to the destination dynamics of system (2.4) as being given by the reduced system (2.5)-(2.7). The specific form for (2.5)-(2.7) may differ for each "attractor" of the original system, but, apart from the initial fast transients, the evolution of the reduced and full systems should be equivalent. In experiment, information on the early, fast evolution may be restricted and the destination dynamics may be all that is available for study. 


\subsubsection{Example: Two Coupled Lorenz Systems}

We consider two Lorenz systems coupled through the variables in the following form:

$$
\begin{aligned}
& \dot{x}_{1}=\sigma\left(x_{2}-x_{4}\right), \\
& \dot{x}_{2}=r x_{1}-x_{2}-x_{1} x_{3}, \\
& \dot{x}_{3}=x_{1} x_{2}-b x_{3}, \\
& \dot{x}_{4}=\sigma\left(x_{5}-x_{4}\right), \\
& \dot{x}_{5}=r x_{1}-x_{5}-x_{1} x_{6}, \\
& \dot{x}_{6}=x_{1} x_{5}-b x_{6},
\end{aligned}
$$

The unidirectional coupling of Lorenz systems, with subsystem $\left(x_{1}-x_{3}\right)$ driving subsystem $\left(x_{4}-x_{6}\right)$, has been studied by Pecora and Carroll, ${ }^{3}$ He and Vaidya, ${ }^{5}$ and Tresser, Worfolk, and Bass $^{6}$ in the context of synchronizing chaos. Such coupling has also been used in schemes for communications. Here, we have also allowed the variable $x_{4}$ to couple the second subsystem back to the first through Eq. (2.8).

Figure 2.3 shows the behavior of this system for three different initial conditions, with parameter values $\sigma=10, r=160$, and $b=8 / 3$. In each case, the system evolves to a different state, with different periodic solutions in (a) and (b) and an aperiodic response in (c). Other initial conditions produce yet different final states for the same parameter values, and the system may also respond to perturbation with a qualitative change in behavior. This gross sensitivity to initial conditions appears to be different from that found in previous studies, where a system exhibits extreme sensitivity in choosing between a small number of attractors for a given set of parameter values.

Figure 2.4(a) shows the largest Lyapunov exponent of the state resulting from initial conditions corresponding to the indicated values of $x_{05}$ and $x_{06}$. The initial values of the other variables, as well as the parameters $\sigma, r$, and $b$, were held constant, and the system was allowed to evolve to its asymptotic state for different $x_{05}$ and $x_{06}$ values. The largest 

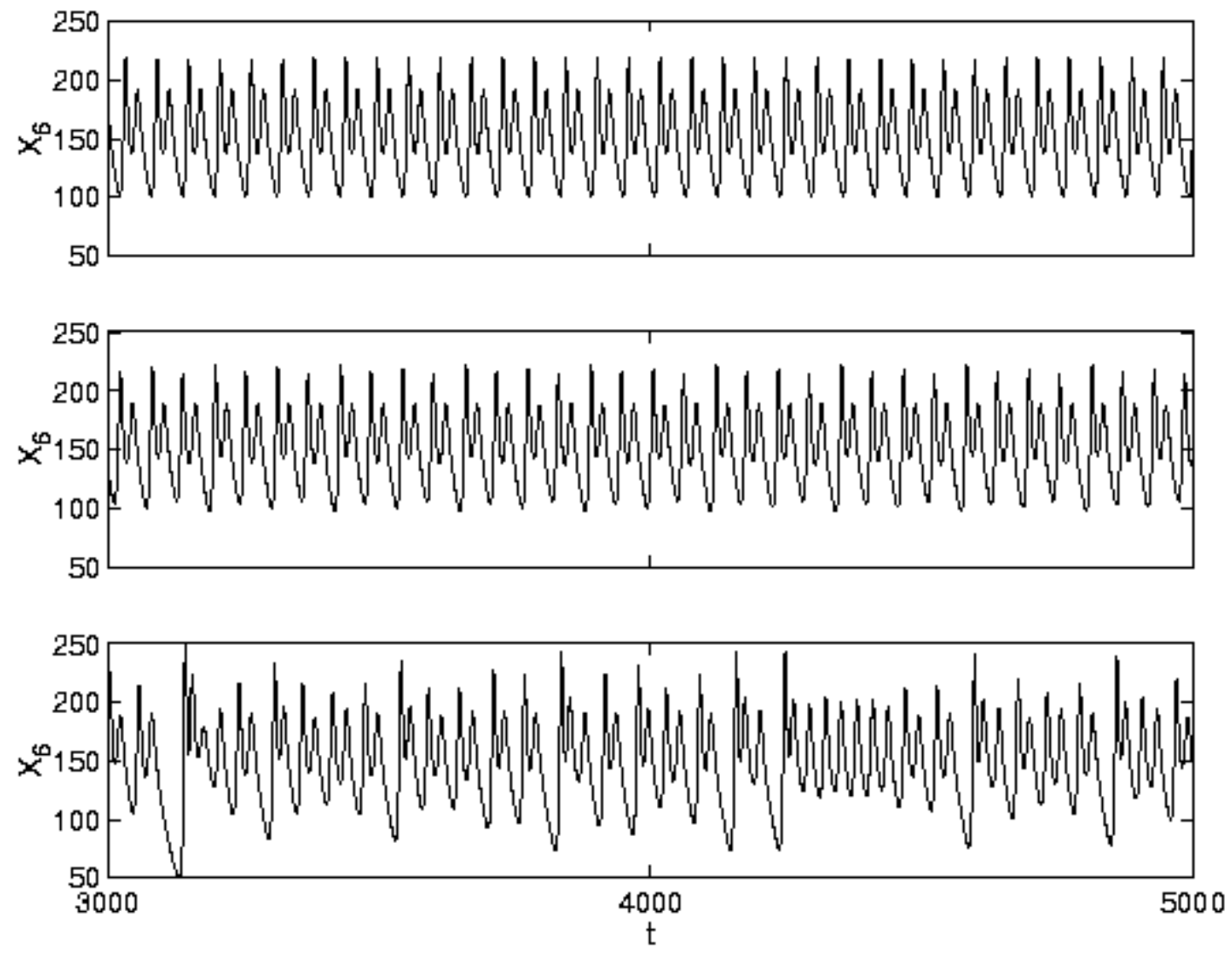

Figure 2.3. Time evolution of Eqs. (2.8)-(2.13) for identical parameter values ( $\sigma=10, b=8 / 3, r=160)$ but for different initial conditions: (a) period $2,\left(x_{05}, x_{06}\right)=(2.0,0.0)$, (b) period $8,\left(x_{05}, x_{06}\right)=(-1.1,1.0)(\mathrm{c})$ chaos, $\left(x_{05}, x_{06}\right)=(0.0,-1.0)$. Other initial conditions: $\left(x_{01}, x_{02}, x_{03}, x_{04}\right)=(0.1,0.02,0.02,1.0)$. 

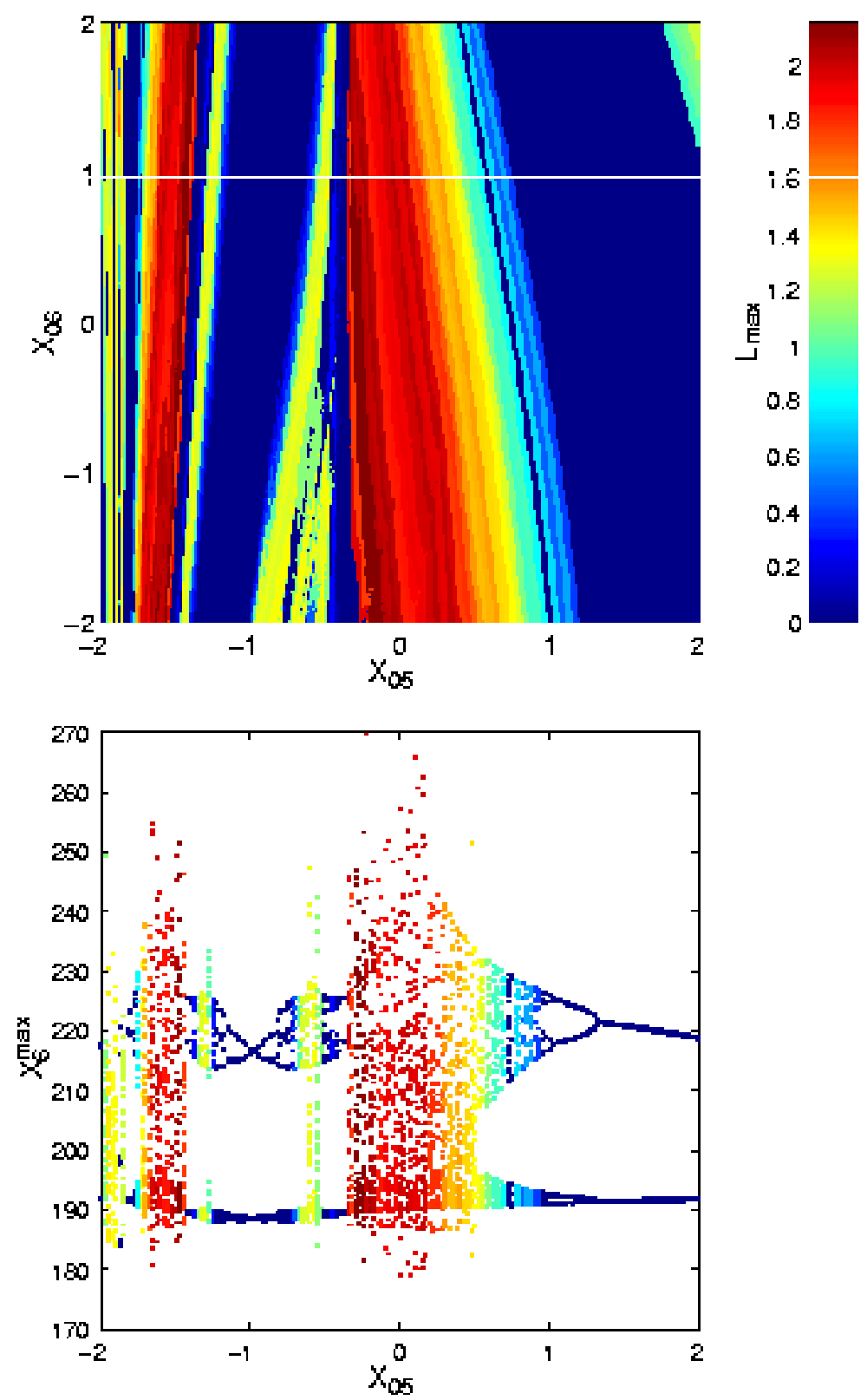

Figure 2.4. (a) Values of the largest Lyapunov exponent corresponding to the state resulting from different initial values $x_{05}$ and $x_{06}$. See side bar for color coding of exponent values. (b) Values of the amplitude of $x_{6}$ as a function of the initial value $x_{05}$ for the initial value $x_{06}=0$. Color coding indicates value of the largest Lyapunov exponent. Parameter values and initial values of other variables as in Fig. 2.3. 
Lyapunov exponent of the corresponding state was then determined and its value was plotted as a function of $x_{05}$ and $x_{06}$ according to the indicated color coding. All periodic states appear as blue, the color assigned to values equal to or less than zero, and chaotic states can be seen as the colors corresponding to positive values. Figure 2.4(b) shows the maximum amplitude of oscillation $x_{6}{ }^{\max }$ as a function of the initial value $x_{05}$ for the same initial values of the other variables. The plot corresponds to a one-dimensional (1D) cut at $x_{06}=0$ of the 2D plot in Fig. 2.4(a), with the color coding again giving the value of the largest Lyapunov exponent.

The dependence on initial conditions shown in Fig. 2.4 suggests that the sixdimensional phase space is foliated with an infinite number of "attractors." To understand this behavior, we consider a reduced system derived from the full system to determine the destination dynamics. Following the method of $\mathrm{He}$ and Vaidya ${ }^{5}$, we construct the governing equations for the "errors" $e_{1}=x_{1}-x_{4}, e_{2}=x_{2}-x_{5}$, and $e_{3}=x_{3}-x_{6}$, giving

$$
\begin{aligned}
& \dot{e}_{1}=\sigma e_{2}, \\
& \dot{e}_{2}=-e_{2}-x_{1} e_{3}, \\
& \dot{e}_{3}=x_{1} e_{2}-b e_{3} .
\end{aligned}
$$

It follows that the function $V=e_{2}^{2}+e_{3}^{2}$ is a Lyapunov function for this system of equations. Thus $e_{2}$ and $e_{3}$ must tend to zero, with the pairs of variables $\left(x_{2}, x_{5}\right)$ and $\left(x_{3}, x_{6}\right)$ becoming absolutely synchronized, independent of the initial conditions. With $e_{2} \rightarrow 0$, then from Eq. (2.14) we also have $e_{1}=\left(x_{1}-x_{4}\right) \rightarrow c$, where $c$ is some constant dependent on the initial conditions of the full system. The system thus establishes a constant difference between the remaining pair of variables $\left(x_{1}, x_{4}\right)$. Each new set of initial conditions gives rise to a different value of $c$ for this difference.

The destination dynamics of Eqs. (2.8)-(2.13) can thus be written as 


$$
\begin{aligned}
& \dot{x}_{1}=\sigma\left(x_{2}-x_{1}+c\right), \\
& \dot{x}_{2}=r x_{1}-x_{2}-x_{1} x_{3}, \\
& \dot{x}_{3}=x_{1} x_{2}-b x_{3},
\end{aligned}
$$

With the constraints

$$
\begin{aligned}
& x_{2}-x_{5}=x_{3}-x_{6}=0, \\
& x_{1}-x_{4}=c .
\end{aligned}
$$

Compared with the traditional Lorenz system, there is now an additional "parameter," namely, the difference $c$ in Eq. (2.17). We can examine the behavior of this system as a function of $c$, and the corresponding bifurcation sequence is illustrated in Fig. 2.5. This sequence is symmetric about $c=0$, as Eqs. (2.17)-(2.19) are invariant under the transformation $\left(x_{1}, x_{2}, c\right) \rightarrow\left(-x_{1},-x_{2},-c\right)$. For $c=0$, the system exhibits a stable period-2 state, but as $|c|$ increases there are various period-doubling and reverse period-doubling cascades with regions of chaos and periodic windows. For the full six-variable system, the value of $c$ corresponding to a given set of initial conditions can be obtained as the long-time limit of the error $e_{1}$. For any such value, the destination dynamics of the corresponding three-variable system can also be computed. We find complete correlation between these two approaches, with only a phase shift remaining at long times.

The change in behavior of the full system with the initial conditions can now be understood in terms of the role played by $c$. The system is comprised of effectively three-dimensional dynamics on invariant manifolds embedded in a four-dimensional space, which is spanned by the "parameter" $c$. For a particular value of $c$, the evolution on the manifold is governed by the "attractor" associated with that value of $c$. This can be clearly seen by comparing the qualitative features of the bifurcation sequence in Fig. 2.5 with the initial conditions sequence in Fig. 2.4(b). Although these sequences do not match quantitatively, since the plot in Fig. 2.4(b) is a projection of behavior along the " $c$ direction," the qualitative correspondence is striking. Perturbing or changing the initial 


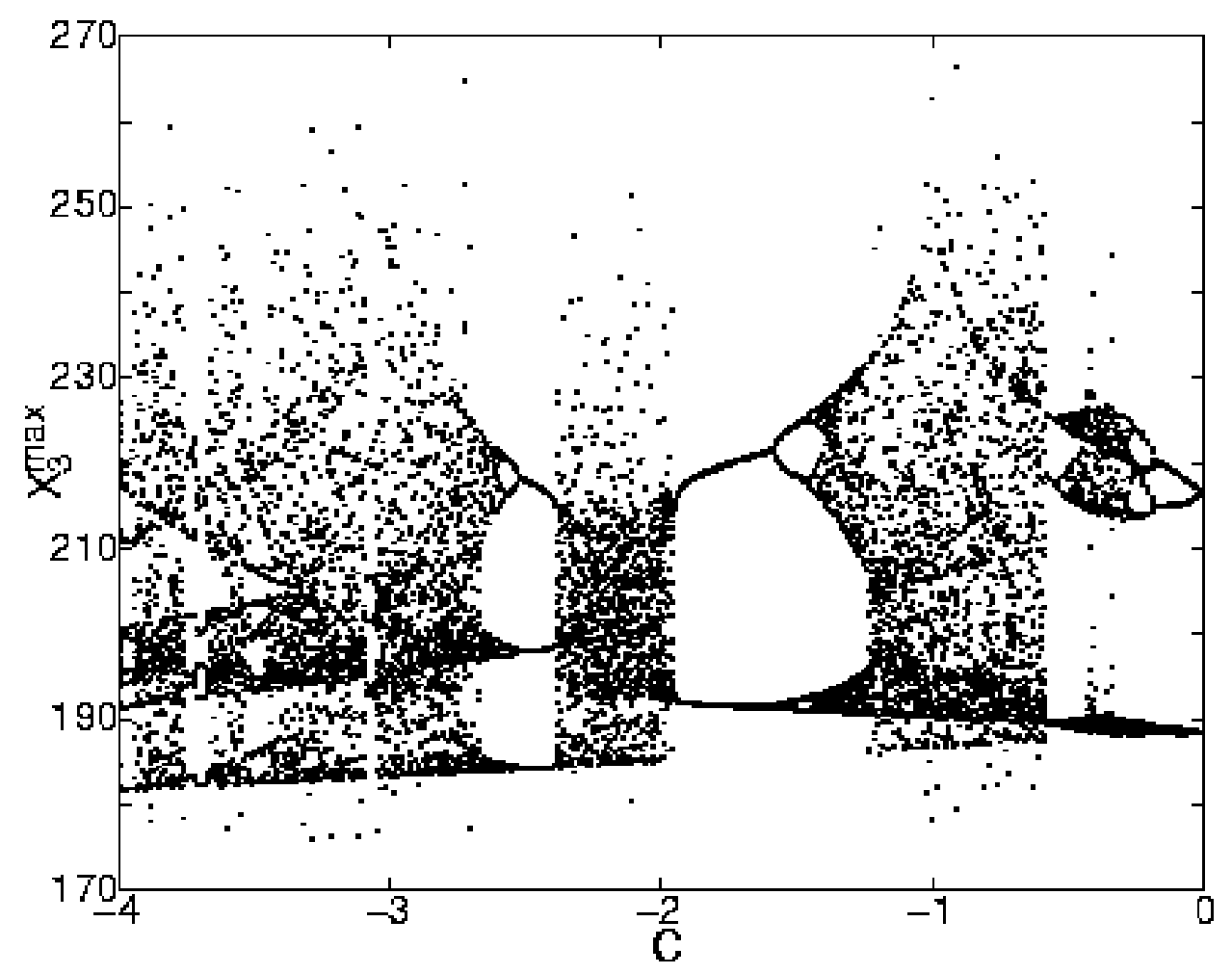

Figure 2.5. Bifurcation sequence of destination dynamics given by Eqs. (2.17)-(2.19) as a function of parameter c. Other parameter values as in Fig. 2.3 . 
conditions of the six-variable system corresponds to shifting the dynamics along the transverse $\mathrm{c}$ direction and hence along the relatively dense bifurcation sequence.

The behavior described above is not unique to the particular model given by Eqs. (2.8)-(2.13). We can readily construct other systems of equations exhibiting uncertain destination dynamics by expanding an appropriate set of reduced equations. For example, as a variation on the Lorenz model, starting with the normal three-variable form [Eqs. (2.17)-(2.19) without the parameter $c$ ], we may replace the term $x_{1} x_{3}$ with a new variable $x_{4}$, which we require to satisfy the condition $x_{4}=x_{1} x_{3}+c$, where $c$ is again some constant. For the latter, we require $d\left(x_{4}-x_{1} x_{3}\right) / d t=0$. The resulting four-variable system also exhibits uncertain destination dynamics.

\subsubsection{Example: A Mechanical System}

As another example, we present a mechanical system, adapted from that advanced by Sommerer and Ott to illustrate riddled basins of attraction. We consider a unit-mass particle moving in $3 \mathrm{D}$ space according to

$$
\frac{d^{2}}{d t^{2}} r=-\gamma \frac{d}{d t} r+F+p \sin \omega t
$$

with $\partial F / \partial t=0$, where $r=(x, y, z)$ and

$$
F=-(\nabla V+\nabla \times A)
$$

consists of a scalar potential field $V$ and a vortex field $A$. Taking, as an example, 


$$
\begin{aligned}
V & =\left(1-x^{2}\right)^{2}, \\
A & =\hat{x} x^{2} z^{2}+\hat{y} x y^{2} z+\hat{z} x z^{3}, \\
p & =p_{0} \hat{x}
\end{aligned}
$$

then

$$
F=\left[4 x\left(1-x^{2}\right)+x y^{2}\right] \hat{x}+\left(z^{3}-2 x^{2} z\right) \hat{y}+\left(-y^{2} z\right) \hat{z}
$$

This then gives the equations

$$
\begin{aligned}
& \frac{d^{2} x}{d t^{2}}=-\gamma \frac{d x}{d t}+4 x\left(1-x^{2}\right)+x y^{2}+p_{0} \sin \omega t, \\
& \frac{d^{2} y}{d t^{2}}=-\gamma \frac{d y}{d t}+\left(z^{2}-2 x^{2}\right) z, \\
& \frac{d^{2} z}{d t^{2}}=-\gamma \frac{d z}{d t}-y^{2} z .
\end{aligned}
$$

This set of equations can be reduced by noting that $z=0$ is a stable manifold, and with $\mathrm{z}$ $\rightarrow 0$ we see from Eq. (2.28) that $d y / d t \rightarrow 0$ and therefore $y \rightarrow c$, where $c$ is a constant. This leads to the reduced system

$$
\frac{d^{2} x}{d t^{2}}=-\gamma \frac{d x}{d t}+4 x\left(1-x^{2}\right)+x c^{2}+p_{0} \sin \omega t
$$

which is of the form of a forced Duffing equation. This is known to have a dense bifurcation structure for suitable parameter combinations and, as this occurs here with a "parameter" $c$ dependent on the initial conditions for the full system, it will show uncertain destination dynamics in a way similar to the coupled Lorenz system, Eqs. (2.8)(2.13). Figure 2.6 shows three examples of the behavior for different initial conditions but with identical parameter values. Again, this result is not specific to the choice of fields, such as Eq. (2.27) taken here, so we expect the behavior to be generic to a wide class of systems. 

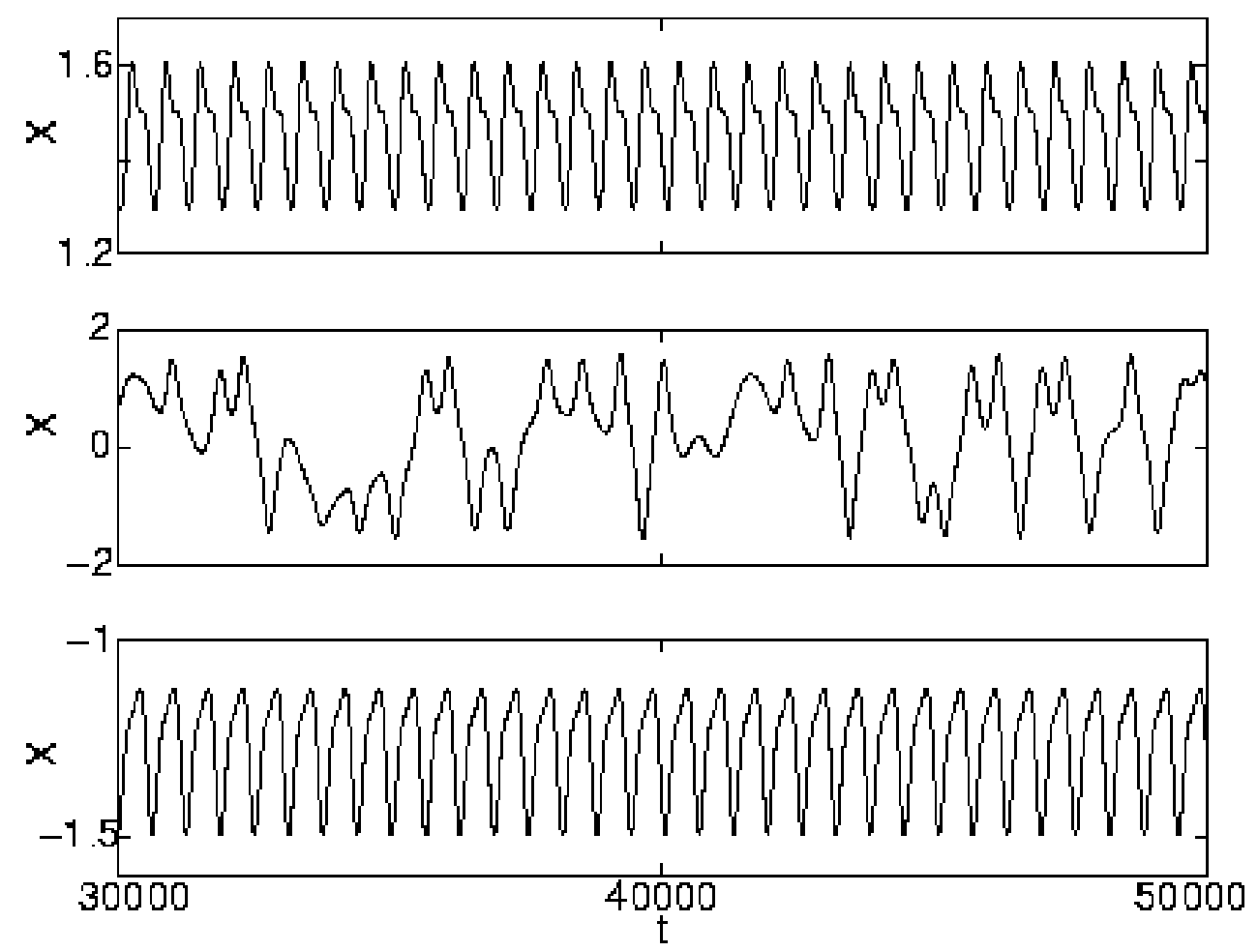

Figure 2.6. Time evolution of Eqs. (11) for identical parameter values $\left(\gamma=0.05, p_{0}=2.3, \omega=3.5\right)$ but with different initial conditions: $\left(x_{0}, v_{x 0}, y_{0}, v_{y 0}, z_{0}, v_{z 0}, t\right)=(0.56,0.0,0.06,0.4,0.3,0.01$, $0.0),(0.56,0.0,-0.26,0.0,0.003,0.0,0.0),(0.56,0.0,0.05,0.0$, $0.03,0.0,0.0)$ in $(\mathrm{a}),(\mathrm{b})$, and (c). 


\subsubsection{Example: Chemical Kinetics}

It has been reported that chemical reactions can exhibit stochastic phenomena ${ }^{32}$, which lead to irreproducibility of experiments no matter how carefully the parameter conditions are prepared. For example, Epstein and Nagypal ${ }^{33}$ reported that, under certain conditions, the chlorite-thiosulfate reaction system exhibits remarkable irreproducibility in the reaction time. They also observed similar phenomena in the chlorite-iodide reaction, where the reaction time can not be reproduced under the supposedly same parameter and initial conditions. In order to explain these experimental observations, Wang et al. ${ }^{34}$ proposed a modified version of the three-variable autocatalator. ${ }^{35,36}$

The proposed model describes a chemically reacting system that converts a reactant $P$ to a final product $D$ through seven steps and five intermediate species $A, B, C, X$, and $Y$, which is given by

$$
\begin{array}{ll}
P \rightarrow A & \text { rate }=k_{p}, \\
P+C \rightarrow A+C & \text { rate }=k_{p c}, \\
A \rightarrow B & \text { rate }=k_{a}, \\
A+2 B \rightarrow 3 B & \text { rate }=k_{a b}, \\
B \rightarrow X+Y & \text { rate }=k_{b}, \\
X+Y \rightarrow C & \text { rate }=k_{x y}, \\
C \rightarrow D & \text { rate }=k_{c} .
\end{array}
$$

This set of reactions is an extension of the three-variable autocatalator model by introducing two new intermediate species, $X$ and $Y$, in the reactions (2.34) and (2.35). In this study, we focus on the case of an open reaction system, in which the chemical precursor $P$ is supplied continuously to allow stable non-equilibrium states.

The dimensionless rate equations governing the evolution of the five intermediate reactants are given by 


$$
\begin{aligned}
& \dot{x}=k-x+\mu z-x y^{2}, \\
& \dot{y}=\sigma^{-1}\left(x-y+x y^{2}\right), \\
& \dot{z}=\delta^{-1}(w v-z), \\
& \dot{w}=y-k_{5} w v, \\
& \dot{v}=y-k_{5} w v,
\end{aligned}
$$

where $k, \mu, \sigma, \delta$, and $k_{5}$ are dimensionless parameters derived from the original reaction equations. From Eqs. (2.40) and (2.41), we notice that the difference between $w$ and $v$ will be constant during the evolution of the dynamical system. The existence of such a constant difference allows us to reduce Eqs. (2.37)-(2.41) to a set of closed four-variable equations by substituting $w=v+c$ in the equations (2.39) and (2.41) and removing Eq. (2.40):

$$
\begin{aligned}
& \dot{x}=k-x+\mu z-x y^{2}, \\
& \dot{y}=\sigma^{-1}\left(x-y+x y^{2}\right), \\
& \dot{z}=\delta^{-1}((v+c) v-z), \\
& \dot{v}=y-k_{5} v(v+c) .
\end{aligned}
$$

Here, $c$ represents the constant difference between $w$ and $v$ in the original system (2.37)(2.41) and is a parameter in the system (2.42)-(2.45). Then, a small perturbation to either $w$ or $v$ may change the constant so that the asymptotic state of the dynamical system may change accordingly. In this model, under certain parameter conditions, the system exhibits dense bifurcations with respect to the constant difference between $w$ and $v$ (Fig. 2.7 and 2.8). Consequently, there is a qualitative change of the asymptotic state, for example, from periodic oscillations to chaos. Therefore, even for two "identical" experiments with the same parameter conditions, the difference between the initial conditions will result in different constants in the destination dynamics and thus qualitatively different asymptotic behavior for the two experiments may occur. This type of model might explain the observed experimental irreproducibility of certain chemical systems. 


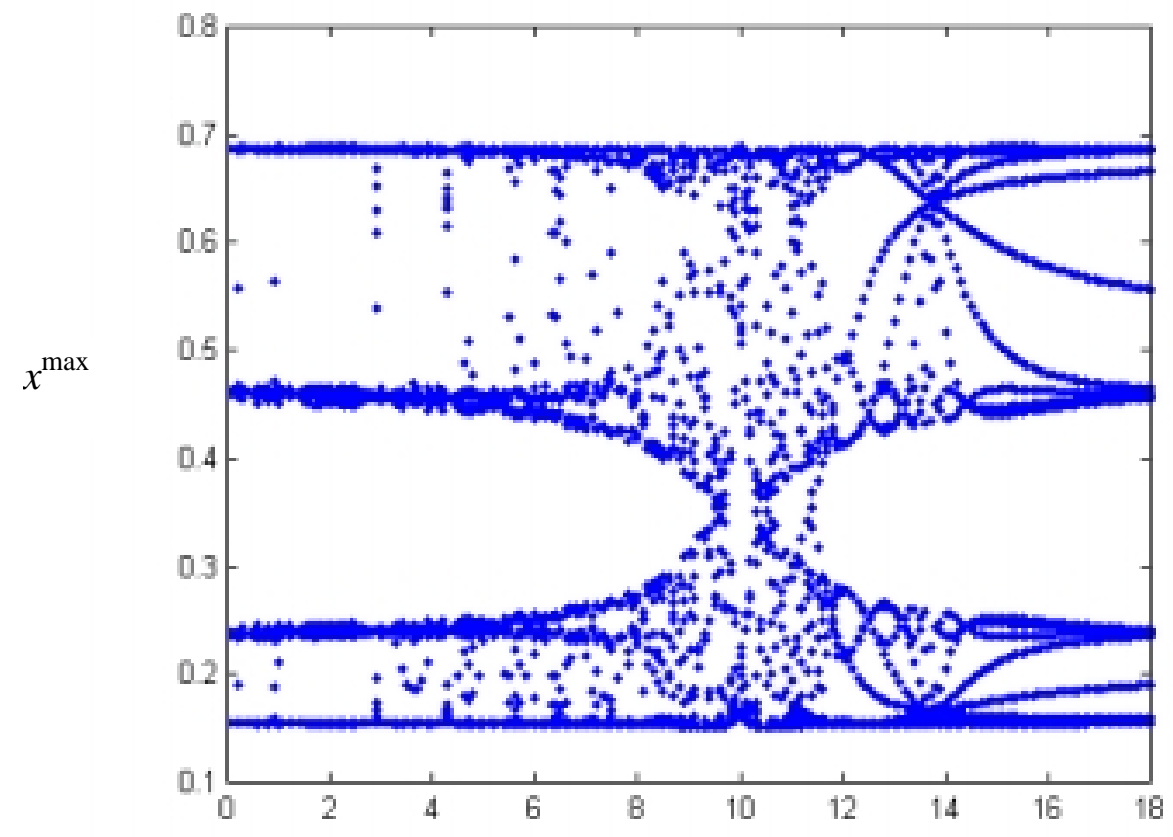

$v_{0}$

Figure 2.7. Bifurcation sequence of values of the amplitude of $x$ as a function of the initial value $v_{0}$ for the initial value $w_{0}=10$ (Eqs. (2.37)2.41). $k=10, \mu=500 / 3, \sigma=1 / 200, \delta=1 / 50, k_{5}=10000 / 9$, and $x_{0}=y_{0}=$ $z_{0}=10^{-5}$. 


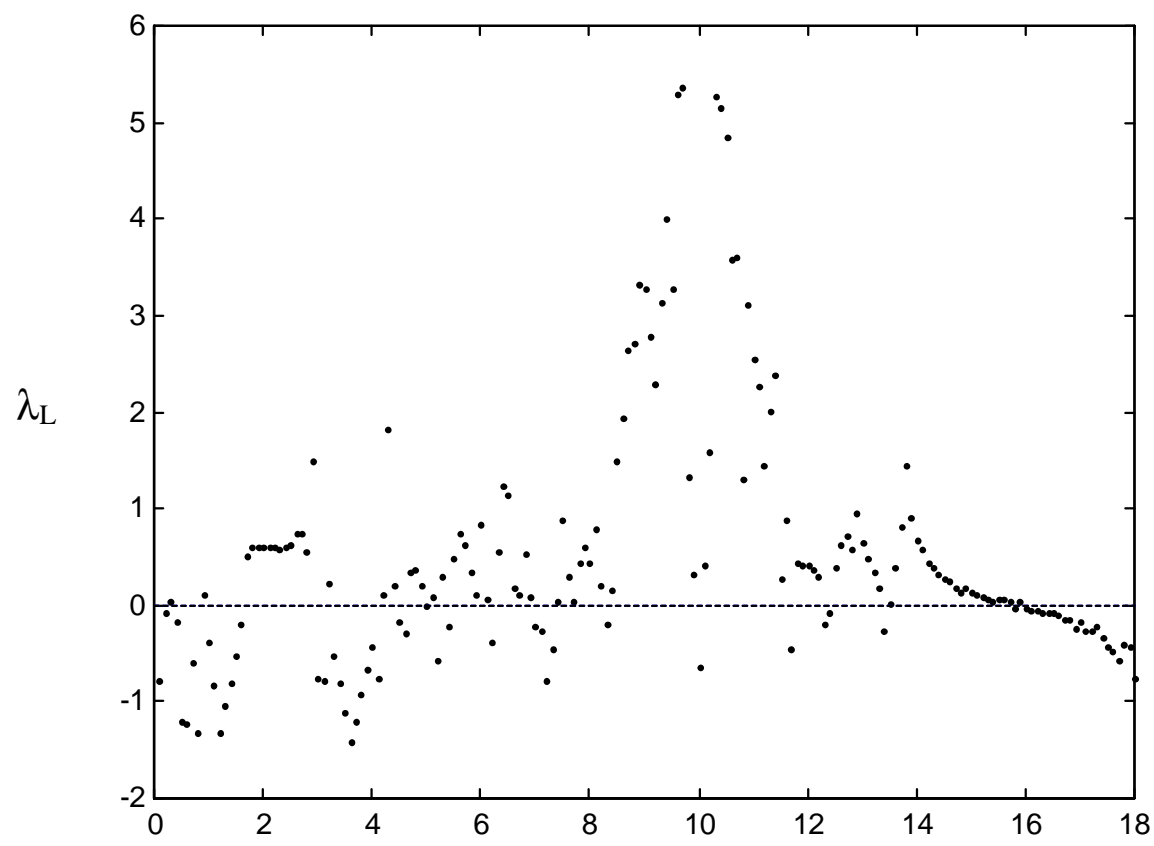

$v_{0}$

Figure 2.8. The largest Lyapunov exponent as a function of the initial value $v_{0}$. Other parameters and initial values are the same as those in Fig. 2.7. 


\subsection{Conclusions}

In summary, we note that in each example of uncertain destination dynamics, a "parameter" $c$ can be identified that attains a particular value following the decay of transient behavior. Different initial conditions or a suitable perturbation give rise to new dynamical behavior corresponding to a different value of $c$. While the behavior is fully dissipative in the manifold corresponding to a particular value of $c$, the system has neutral stability to influences that move it transverse to the manifold to a new value of $c$. It is this neutral stability in the " $c$ direction" that gives rise to the foliation of "attractors" in phase space and the corresponding dependence on initial conditions. 


\subsection{Appendix: The Largest Lyapunov Exponent}

The largest Lyapunov exponent is a measure of dynamical complexity. For a deterministic dynamical system, if it exhibits chaotic behavior, the largest Lyapunov exponent is positive.

In the case of a continuous dynamical system defined by a set of ordinary differential equations,

$$
\frac{d x(t)}{d t}=F(x(t))
$$

one can differentiate to obtain

$$
\frac{d u(t)}{d t}=\left(D_{x(t)} F\right)[u(t)]
$$

which is linear in $u$, but with non-constant coefficients. The solution of (A1) yields

$$
x(t)=f^{t}(x(0))
$$

and the solution of (A2) yields

$$
u(t)=D_{x(0)} f^{t} u(0) .
$$

Therefore, one can readily compute the matrices $T_{x}{ }^{t}=D_{x} f^{t}$ and the largest Lyapunov exponent by

$$
\lambda_{L}=\lim _{t \rightarrow \infty} \frac{1}{t} \log \left\|T_{x}^{t} u\right\|
$$




\subsection{References}

1. H. Fujisaka and T. Yamada, Prog. Theor. Phys. 74, 919 (1985).

2. A. V. Gaponov-Grekhov, M. I. Rabinovich, and I. M. Starobinets, Pis'ma v Zh. Eksp. Theor. Fiz 39, 561 (1984).

3. L. M. Pecora and T. L. Carroll, Phys. Rev. Lett. 64, 821 (1990).

4. L. M. Pecora and T. L. Carroll, G. A. Johnson, D. J. Mar, and J. F. Heagy, Chaos 7, 520 (1997).

5. C. Tresser, P. A. Worfolk, and H. Bass, Chaos 5, 693 (1995).

6. R. He and P. G. Vaidya, Phys. Rev. A 46, 7387 (1992).

7. K. M. Cuomo and A. V. Oppenheim, Phys. Rev. Lett. 71, 65 (1993).

8. E. A. Spiegel, Ann. (N.Y.) Acad. Sci. 6117, 305 (1981).

9. A. S. Pikovsky, Z. Phys. B 55, 149 (1984).

10. N. Platt, E. A. Spiegel, and C. Tresser, Phys. Rev. Lett. 70, 279 (1993).

11. J. F. Heagy, N. Platt, and S. M. Hammel, Phys. Rev. E 49, 1140 (1994).

12. L. Yu, E. Ott, and Q. Chen, Phys. Rev. Lett. 65, 2935 (1990).

13. S. C. Venkataramani, T. M. Antonsen, Jr., E. Ott, and J. C. Sommerer, Phys. Lett. A 207, 173 (1995).

14. J. C. Alexander, I. Kan, J. A. Yorke, and Z. You, Int. J. Bifurcation Chaos Appl. Sci. Eng. 2, 795 (1992).

15. J. C. Sommerer and E. Ott, Nature (London) 365, 136 (1993).

16. E. Ott and J. C. Sommerer, Phys. Lett. A 188, 39 (1994).

17. E. Ott, J. C. Alexander, I. Kan, J. C. Sommerer, and J. A. Yorke, Physica (Amsterdam) 76D, 384 (1994).

18. I. Kan, Bull. Am. Math. Soc. 31, 68 (1994). 
19. J. C. Sommerer and E. Ott, Phys. Lett. A 214, 243 (1996).

20. J. F. Heagy, T. L. Carroll, and L. M. Pecora, Phys. Rev. Lett. 73, 3528 (1994).

21. Y. C. Lai and R. L. Winslow, Phys. Rev. Lett. 72, 1640 (1994).

22. Y. C. Lai, C. Grebogi, and E. J. Kostelich, Phys. Lett. A 196, 206 (1994).

23. Y. C. Lai and C. Grebogi, Phys. Rev. Lett. 77, 5047 (1996).

24. T. Sauer, C. Grebogi, and J. A. Yorke, Phys. Rev. Lett. 79, 59 (1997).

25. J. Guckenheimer and P. Holmes, Nonlinear Oscillations, Dynamical Systems, and Bifurcations of Vector Fields, Springer-Verlag, New York, 1983.

26. R. L. Devaney, An Introduction to Chaotic Dynamical Systems, Benjamin/Cummings, Menlo Park, 1985.

27. S. H. Strogatz, Nonlinear Dynamics and Chaos, Addison-Wesley, New York, 1994.

28. T. S. Parker and L. O. Chua, Practical Numerical Algorithms for Chaotic Systems, Springer-Verlag, New York, 1989.

29. H. Sun, S. K. Scott, and K. Showalter, Phys. Rev. E 60, 3876 (1999).

30. E. N. Lorenz, J. Atmos. Sci. 20, 130 (1963).

31. C. Sparrow, The Lorenz Equations: Bifurcations, Chaos and Strange Attractors, Springer-Verlag, New York, 1982.

32. S. M. Zoldi and H. S. Greenside, Phys. Rev. Lett. 78, 1687 (1997).

33. I. Epstein and E. Nagypal, to be published.

34. J. Wang, H. Sun, and K. Showalter, in preparation.

35. B. Peng, S. K. Scott, and K. Showalter, J. Phys. Chem. 94, 5243 (1990).

36. S. K. Scott, B. Peng, and K. Showalter, J. Chem. Phys. 94, 1134 (1991). 


\section{Chapter 3}

\section{Synchronization of Mutually Coupled Dynamical Systems}

\subsection{Introduction}

Chaos in a nonlinear dynamical system generates disordered behavior, while synchronization is a mechanism of suppressing the disorder of coupled chaotic systems because, through synchronization, the number of degrees of freedom of the dynamical system is reduced.

The study of synchronization can be dated back to year 1673, when Huygens discovered that two single pendula can couple to oscillate with the same amplitude and phase. ${ }^{1}$ In the classical sense, synchronization means adjustment or entrainment of frequencies of periodic oscillators due to a weak interaction. ${ }^{2-4}$ This effect has since been well studied and applied to various fields. ${ }^{5-7}$ On the other hand, the study of chaos has developed to a stage where the coupling of chaotic systems has attracted increasing attention. Consequently, the study of chaos synchronization has become a natural extension of the theory of dynamics and considerable progress has been made since its advent in the 1980 s. $^{8-10}$ Because chaos involves extreme sensitivity to initial conditions, any small perturbation leads to an exponential divergence between trajectories. It therefore seemed surprising to achieve synchronization of chaotic systems. However, in 1990, Pecora and Carroll $^{11}$ experimentally demonstrated the existence of chaos 
synchronization, using an electronic circuit coupled unidirectionally to a subsystem made up of components of the parent system. They also showed theoretically that this process can be achieved when the sub-Lyapunov exponents for the subsystem to be synchronized are all negative. After this discovery, Cuomo and Oppenheim further expanded the area by demonstrating the potential application of chaos synchronization for private communication. ${ }^{12}$ In the same period, various experiments besides those in electronics were conducted by different groups, for example, experiments demonstrating chaos synchronization in coupled laser systems. ${ }^{13,14}$ However, coupling schemes with both mutual and multiplicative character have lacked investigation, despite their broad existence in dynamical systems.

Beyond the study of chaos synchronization in ODE systems, spatiotemporal synchronization has recently become an important research focus. ${ }^{15-17}$ Synchronizing complex spatiotemporal patterns is extremely challenging because the number of degrees of freedom of an extended system can be infinite. However, various approximation methods exist for us to simplify such complex systems. In order to help understand the methods used in the study of spatiotemporal synchronization, we list the characteristics of different spatiotemporal systems in the following table:

Table 3.1. Characters of spatiotemporal systems

\begin{tabular}{|c|c|c|c|}
\hline Dynamical System & Time & Space & State/Variable \\
\hline Cellular Automata & Discrete & Discrete & Discrete \\
\hline Coupled Map Lattice & Discrete & Discrete & Continuous \\
\hline Coupled ODE Lattice & Continuous & Discrete & Continuous \\
\hline PDE & Continuous & Continuous & Continuous \\
\hline
\end{tabular}

The above four spatiotemporal systems have been the most studied, although we can have 8 combinations based on the differences in time, space, and state. Among them, cellular automata (CA) have the least number of degrees of freedom, coupled map lattice (CML) 
and coupled ODE lattice (COL) are next, while a PDE system has the most number of degrees of freedom, namely infinity. This classification is based on the original forms of the equations rather than their representations in numerical simulations (with the aid of a digital computer, all systems are equivalent to CAs or, at best, CMLs). Recently, Roman et al. conducted numerical studies of spatiotemporal synchronization using CMLs; ${ }^{18}$ Zanette et al. ${ }^{19}$ and Braiman et al. ${ }^{16}$ employed COL; Boccaletti et al. ${ }^{17}$ presented an example of a PDE system, namely the complex Ginzburg-Landau equation. Each study successfully achieved spatiotemporal synchronization and even chaotic spatiotemporal synchronization. In regard to the coupling schemes used in these studies, we note that linearly additive couplings rather than multiplicative ones prevail, although mutual couplings are always used in the spatiotemporal systems.

We focus on investigating synchronous phenomena in coupled dynamical systems with both mutual and multiplicative couplings existing simultaneously. Following the introduction, we classify various types of chaos synchronization in Section 3.2. In Section 3.3 and 3.4, we present our studies in coupled ODE and PDE systems, respectively. In Section 3.5, we draw our conclusions.

\subsection{Classification of chaos synchronization}

Chaos synchronization can be classified based on two different criteria, either the asymptotic state or the coupling scheme. In the following graphs, we list only wellestablished concepts and some closely related concepts. Only representative references are given rather than an exhaustive bibliography. Synchronization of hyperchaos, ${ }^{20,21}$ exhibited in systems having at least two positive Lyapunov exponents, can also be classified in a similar way. 


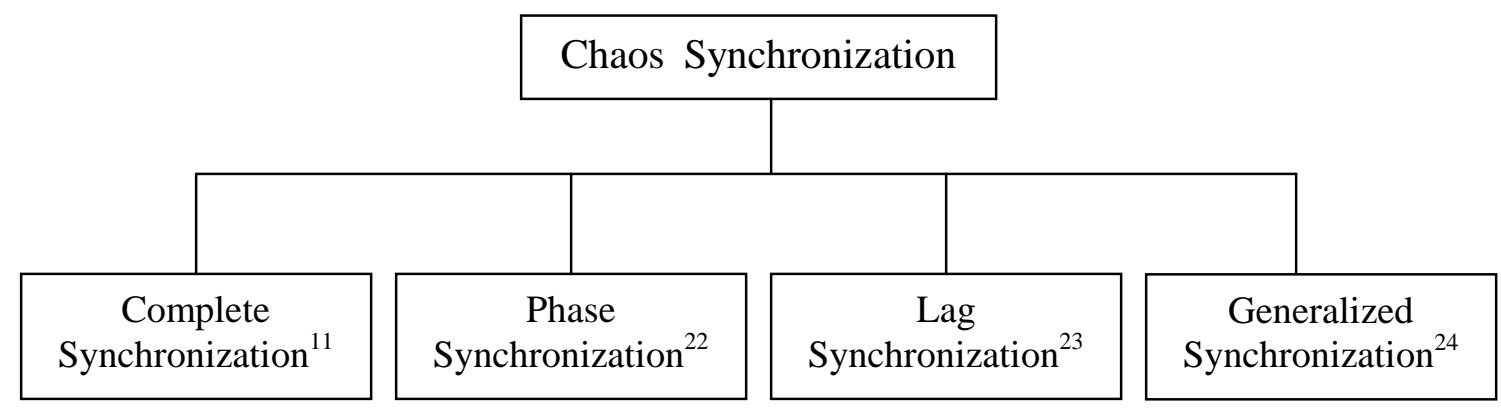

Figure 3.1. Classification of chaos synchronization based on asymptotic states.

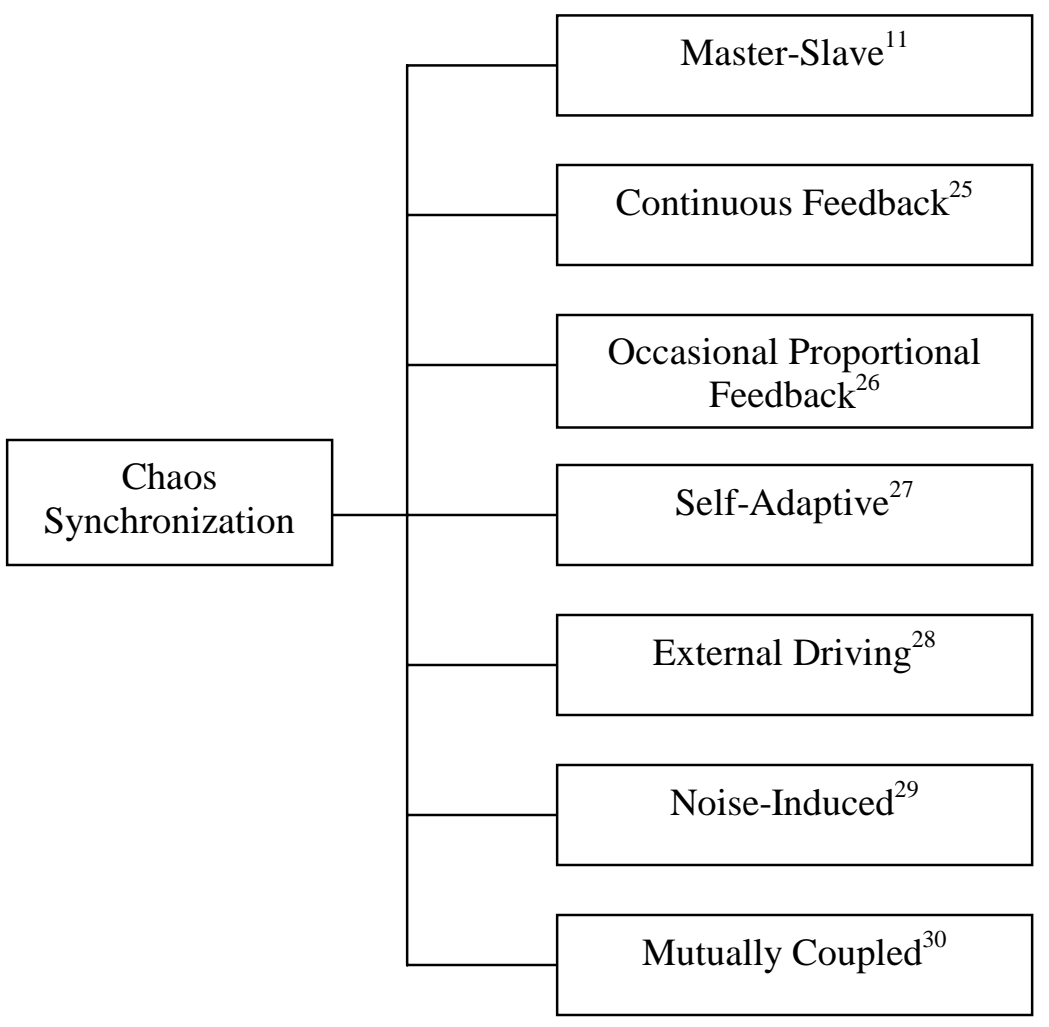

Figure 3.2. Classification of chaos synchronization based on coupling schemes. 


\subsection{Synchronization in an ODE system}

In Chapter 2, we discussed the possibility of uncertainty in high-dimensional nonlinear dynamical systems. The first example there, the mutually coupled Lorenz systems, ${ }^{31}$ can also be regarded as an example showing chaos synchronization. This type of synchronization has a special feature: Depending on the initial conditions, the asymptotic synchronization involves one system $\left(x_{1}, x_{2}, x_{3}\right)$ undergoing a shift relative to another system $\left(y_{1}, y_{2}, y_{3}\right)$ in the phase space. The distance for such a shift in phase space is characterized by the "uncertainty constant" $c$, which depends on the initial conditions (Fig. 3.3).

Here, we provide another example based on the Lorenz model, which exhibits stable synchronization and shows that one subsystem is an expansion or contraction of another subsystem in phase space. The equations are

$$
\begin{aligned}
& \dot{x}_{1}=\sigma\left(x_{2}-x_{1}\right), \\
& \dot{x}_{2}=r x_{1}-x_{2}-x_{1} y_{3}, \\
& \dot{x}_{3}=x_{1} x_{2}-b y_{3}, \\
& \dot{y}_{1}=\sigma\left(y_{2}-y_{1}\right), \\
& \dot{y}_{2}=r y_{1}-y_{2}-y_{1} y_{3}, \\
& \dot{y}_{3}=y_{1} x_{2}-b y_{3},
\end{aligned}
$$

where $\sigma, r$ and $b$ are parameters. Referring to the original Lorenz model, we select $x_{2}$ and $y_{3}$ to be the mutual coupling variables: $x_{2}$ partially substitutes for $y_{2}$ in the $y$-subsystem and $y_{3}$ is fed back into the $x$-subsystem to replace $x_{3}$. The multiplicative couplings lie in the two products, $x_{1} y_{3}$ and $y_{1} x_{2}$. In this model, $x_{3}$ is not an essential variable because it is driven by $x_{1}, x_{2}$, and $y_{3}$ but has no feedback couplings to the other variables. We retain $x_{3}$ in the equations to maintain the symmetry but focus on the evolution of $x_{1}, x_{2}, y_{1}$, and $y_{2}$. The evolution equation of $y_{3}$ gives rise to the main coupling for the subsystems $\left(x_{1}, x_{2}\right)$ and $\left(y_{1}, y_{2}\right)$. 

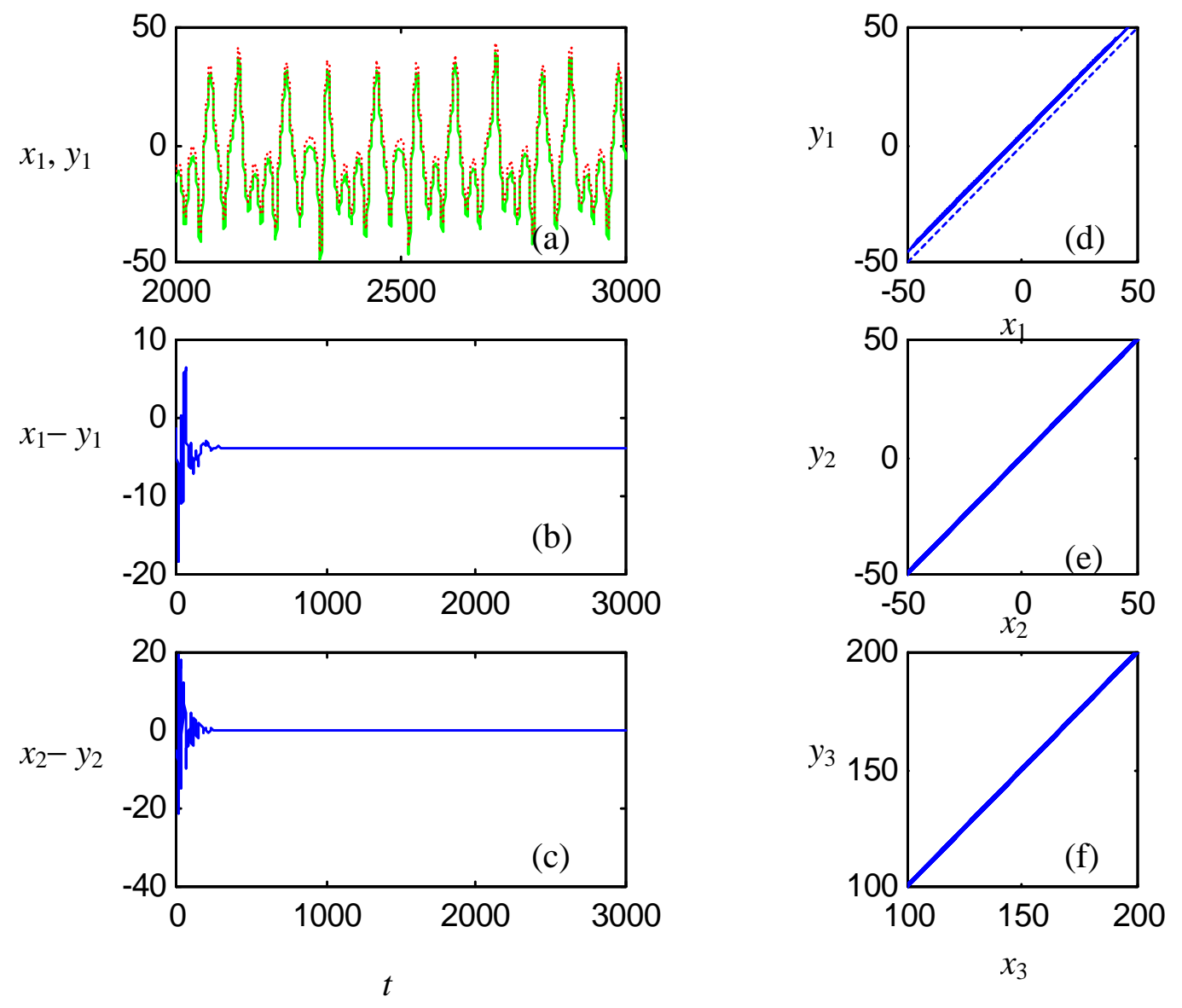

Figure 3.3. Illustration of the "shift" synchronization in the coupled Lorenz system (see Chapter 2, Eq. (2.5)). $\sigma=10, \mathrm{r}=160, \mathrm{~b}=8 / 3$. Initial conditions are $\left(x_{1}, x_{2}, x_{3}\right)=(0.1,0.02,0.02)$ and $\left(y_{1}, y_{2}, y_{3}\right)=(1.0,5.0$, 30.0). In (a), $x_{1}$ and $y_{1}$ are represented by a solid line and a dotted line, respectively. The dashed line in (d) corresponds to the diagonal. 
The system can be analyzed based on the following relations derived from Eq. (3.1)

$$
\begin{aligned}
& \frac{d}{d t}\left(\frac{x_{1}}{y_{1}}\right)=\frac{\dot{x}_{1} y_{1}-x_{1} \dot{y}_{1}}{y_{1}{ }^{2}}=\frac{\sigma\left(x_{2} y_{1}-x_{1} y_{2}\right)}{y_{1}{ }^{2}}, \\
& \frac{d}{d t}\left(\frac{x_{2}}{y_{2}}\right)=\frac{\dot{x}_{2} y_{2}-x_{2} \dot{y}_{2}}{y_{2}{ }^{2}}=\frac{\left(y_{3}-r\right)\left(x_{2} y_{1}-x_{1} y_{2}\right)}{y_{2}{ }^{2}} .
\end{aligned}
$$

If $y_{1}=c x_{1}$ and $y_{2}=c x_{2}$ are substituted into the above equations (where $c$ is a constant), both $d\left(x_{1} / y_{1}\right) / d t$ and $d\left(x_{2} / y_{2}\right) / d t$ vanish. This demonstrates the existence of synchronous solutions in the coupled systems, and since $c$ is unspecified, it can take on any value. Then, how does the system evolve to the synchronous state? We note that the variables of interest have coupled motion in the phase space, which means $d\left(x_{1} / y_{1}\right) / d t=0$ at some instance when $x_{1} / y_{1}$ reaches an extremum. It follows that $x_{2} y_{1}=x_{1} \mathrm{y}_{2}$, i.e., $x_{2} / y_{2}=x_{1} / y_{1}$, according to Eq.(3.2). This also explains why the slopes in the plots of $x_{1}$ vs. $y_{1}$ and $x_{2}$ vs. $y_{2}$ must be the same to achieve synchronization. Fig. 3.4 (a) shows the evolution of the variables $x_{1}$ (solid line) and $y_{1}$ (dotted line) of Eq. (3.1). In Fig. 3.4 (b), we show the absolute difference between $x_{1}$ and $y_{1}$, which demonstrates that the difference will never vanish and, in fact, it exhibits chaotic oscillations similar to $x_{1}$ or $y_{1}$. However, if we plot $x_{1}-c y_{1}(c \sim 3.42)$, as shown in Fig. 3.4 (c), the difference quickly disappears, which means $x_{1}$ is a proportional expansion of $y_{1}$. The off-diagonal straight lines in Fig. 3.4 (d) and (e) demonstrate that the synchronization is phase synchronization rather than complete synchronization. Upon synchronization, the coupled subsystems are reduced to a set of 3-variable equations

$$
\begin{aligned}
& \dot{y}_{1}=\sigma\left(y_{2}-y_{1}\right), \\
& \dot{y}_{2}=r y_{1}-y_{2}-y_{1} y_{3}, \\
& \dot{y}_{3}=c y_{1} y_{2}-b y_{3},
\end{aligned}
$$



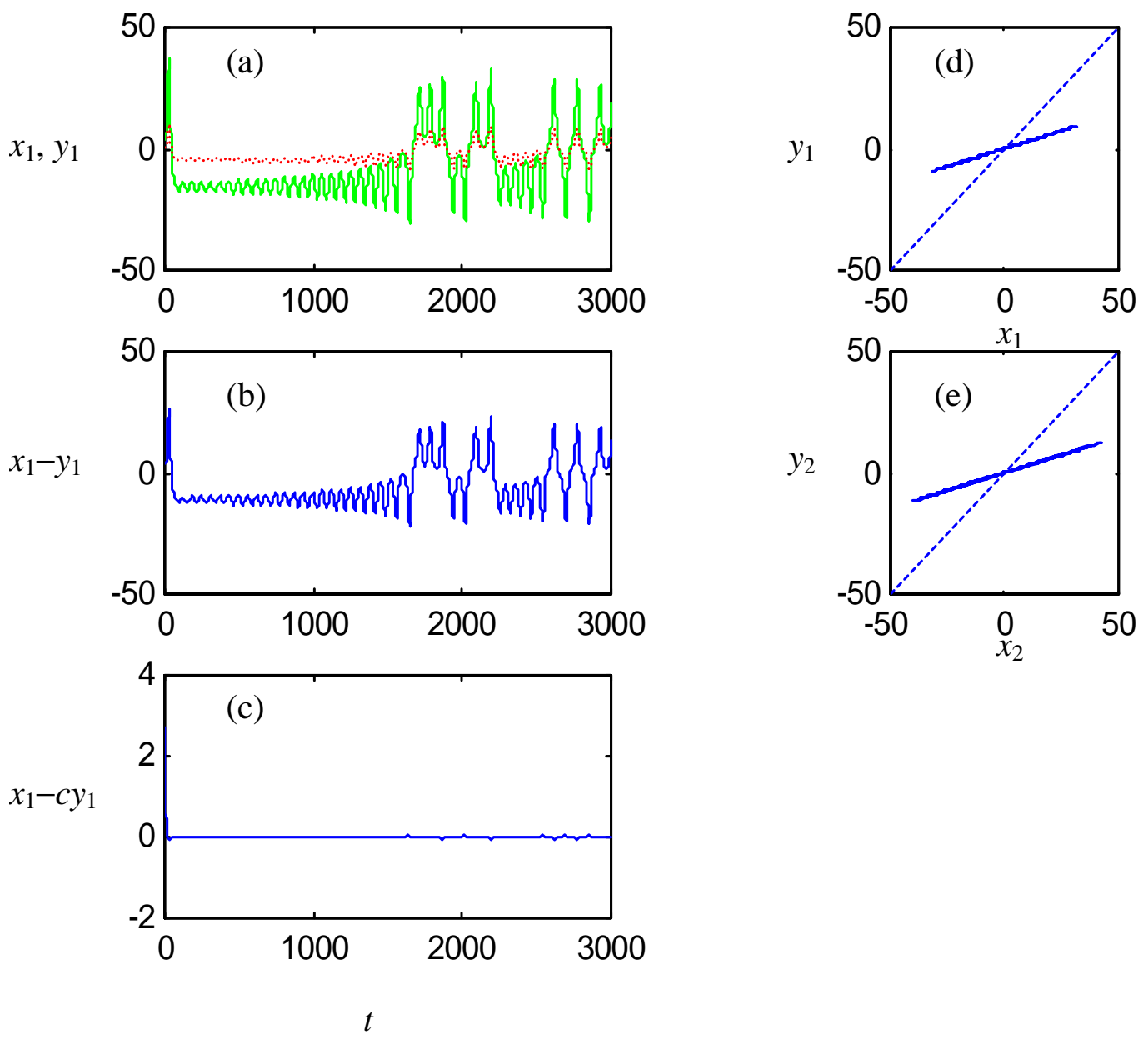

Figure 3.4. Illustration of the "expansion" synchronization in the coupled Lorenz system (Eq. (3.1)). $\sigma=10, \mathrm{r}=28, \mathrm{~b}=8 / 3$. Initial conditions are $\left(x_{1}, x_{2}, x_{3}\right)=(3.5,3.5,0.62)$ and $\left(y_{1}, y_{2}, y_{3}\right)=(0.02,2.3,0.54) . c$ is numerically determined to be 3.42. In (a), $x_{1}$ and $y_{1}$ is represented by a solid line and a dotted line, respectively. 
with the algebraic constraints $x_{1}=c y_{1}$ and $x_{2}=c y_{2}$. The equations are a modified version of the Lorenz model and exhibit chaotic behavior under suitable parameter conditions. The constant $c$ depends on initial conditions of the full system, Eq. (3.1), and determines the ratio between the amplitudes of the two oscillators.

It is interesting to note that this model can be expanded to multiple coupled chaotic oscillators as shown in scheme Fig. 3.5, where the central oscillator $\left(y_{1}, y_{2}, y_{3}\right)$ can be considered as a hub for the synchronous network. Upon synchronization, $y_{2}=x_{2}=z_{2}=u_{2}$ $=\ldots$, the central oscillator is once again determined by Eq. (3.3), with the constant $c$ having contributions from all other oscillators. This extension has a potential for developing multiple-party private communications.

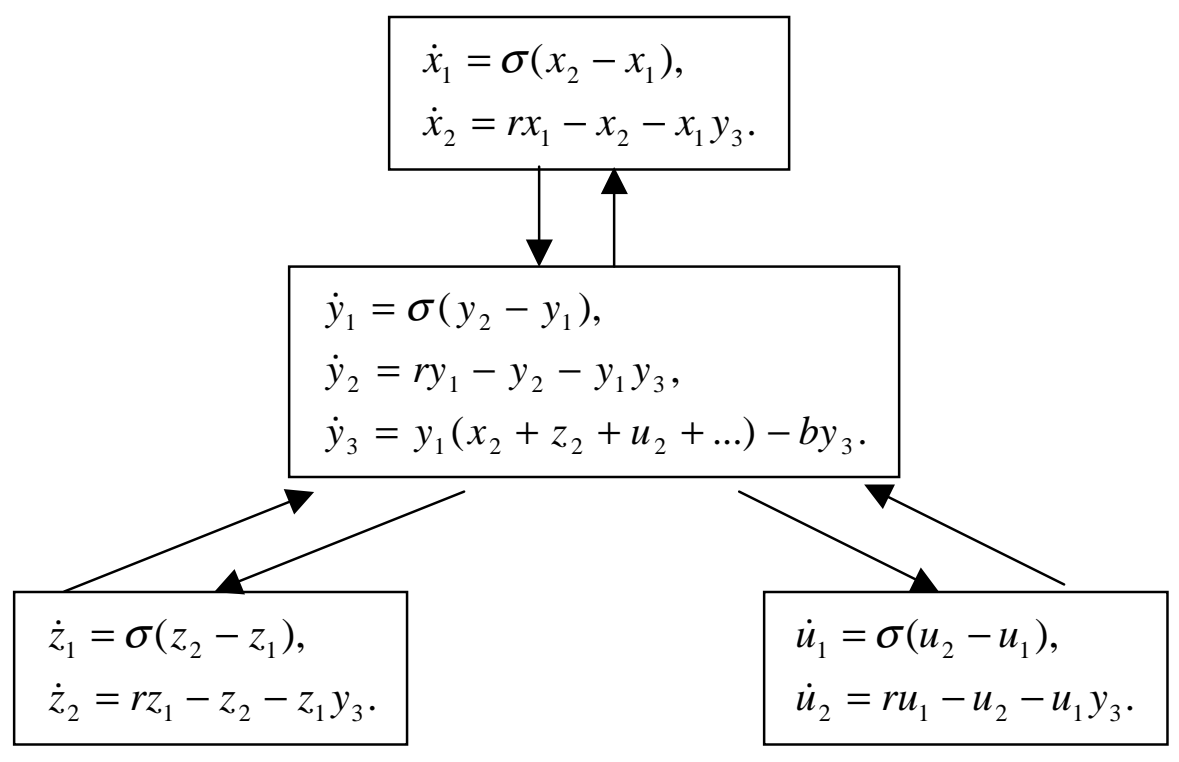

Figure 3.5. Illustration of multiple coupled systems for chaos synchronization. 


\subsection{Synchronization in a PDE system}

\subsubsection{The Barkley model}

Systems undergoing chemical reactions show numerous pattern forming phenomena which combine hydrodynamics with the molecular reactions. ${ }^{32}$ Many spatial patterns occurring in hydrodynamics and in thermodynamic phase formations are strongly influenced by chemical processes ${ }^{33}$ and various types of catalytic reactions. ${ }^{34}$ In recent years, there has been a growing interest in the subject of sustained oscillations in reaction-diffusion systems, which was sparked by the classic Belousov-Zhabotinsky (BZ) reaction. ${ }^{35,36}$ This experimental system exhibited excitability in a well-stirred chemical reactor: Under different experimental conditions (concentrations, temperature, etc.), the system is stable in either the oxidized state or reduced state and, alternatively, the system can be bistable, so that for fixed parameter values it can go to either state depending on initial conditions. In both the stable and bistable cases, the system can be excitable, which means that certain initial conditions decay rapidly to the stable state, while others lead to large deviations before the system eventually reaches a stable state.

A classic model describing the BZ system is the Oregonator ${ }^{37}$, and a dimensionless version of the model is given by

$$
\begin{aligned}
& \frac{\partial u}{\partial t}=\varepsilon^{-1} f(u, v)+D_{u} \nabla^{2} u \\
& \frac{\partial v}{\partial t}=g(u, v)+D_{v} \nabla^{2} v
\end{aligned}
$$

where $\varepsilon$ is a parameter and $D_{u}$ and $D_{v}$ are the diffusion coefficients of $u$ and $v$, respectively. The functions $f$ and $g$ can have different versions, for example, $f(u, v)=$ $u(1-u)-b v(u-a) /(u+a)$ and $g(u, v)=u-v$ as used by Tyson and Fife. ${ }^{38}$ However, the time scale on which the variables change as the system becomes locally excited is typically 
several orders of magnitude faster than the time scale on which interesting behavior occurs in the extended medium. In 1991, Barkley proposed a new computationally efficient model for excitable media without sacrificing any essential properties of the dynamics. ${ }^{39,40}$ The Barkley model is written as

$$
\begin{aligned}
& \frac{\partial u}{\partial t}=f(u, v)+\nabla^{2} u, \\
& \frac{\partial v}{\partial t}=g(u, v),
\end{aligned}
$$

where

$$
\begin{aligned}
& f(u, v)=\varepsilon^{-1} u(1-u)\left[u-\frac{v+b}{a}\right], \\
& g(u, v)=u-v,
\end{aligned}
$$

and $a, b$, and $\varepsilon$ are positive parameters, with $\varepsilon$ generally small. Here, the diffusion coefficient for the $u$-variable is scaled to unity and, for simplicity, $v$ is assumed not to diffuse. The variables $u$ and $v$ are known as the excitation and recovery/inhibition variables, respectively, and the system has a stable but excitable fixed point at the intersect of the nullclines, $f(u, v)=0$ and $g(u, v)=0$. In Fig. 3.6, we illustrate the local dynamics of the system by analyzing the system nullclines. The $v$ nullcline $g(u, v)=0$ is the line $v=u$, and the $u$ nullcline $f(u, v)=0$ consists of three lines: $u=0, u=1$, and $u=$ $(v+b) / a$. An excitable fixed point is located at the origin where the $u$ and $v$ nullclines intersect. $u=(v+b) / a$ is the excitable threshold for the fixed point. Initial conditions near the fixed point and to the left of the threshold decay directly to the fixed point. Initial conditions to the right of the threshold experience a large excursion before returning to the fixed point. The system remains longer near the nullcline branches $u=0$ and $u=1$, while it undergoes fast dynamics ${ }^{41}$ between these branches. This relaxation dynamics of the Barkley model makes it useful for studying spatial patterns. Fig. 3.7 shows a simulation of spiral wave behavior with the Barkley model. 


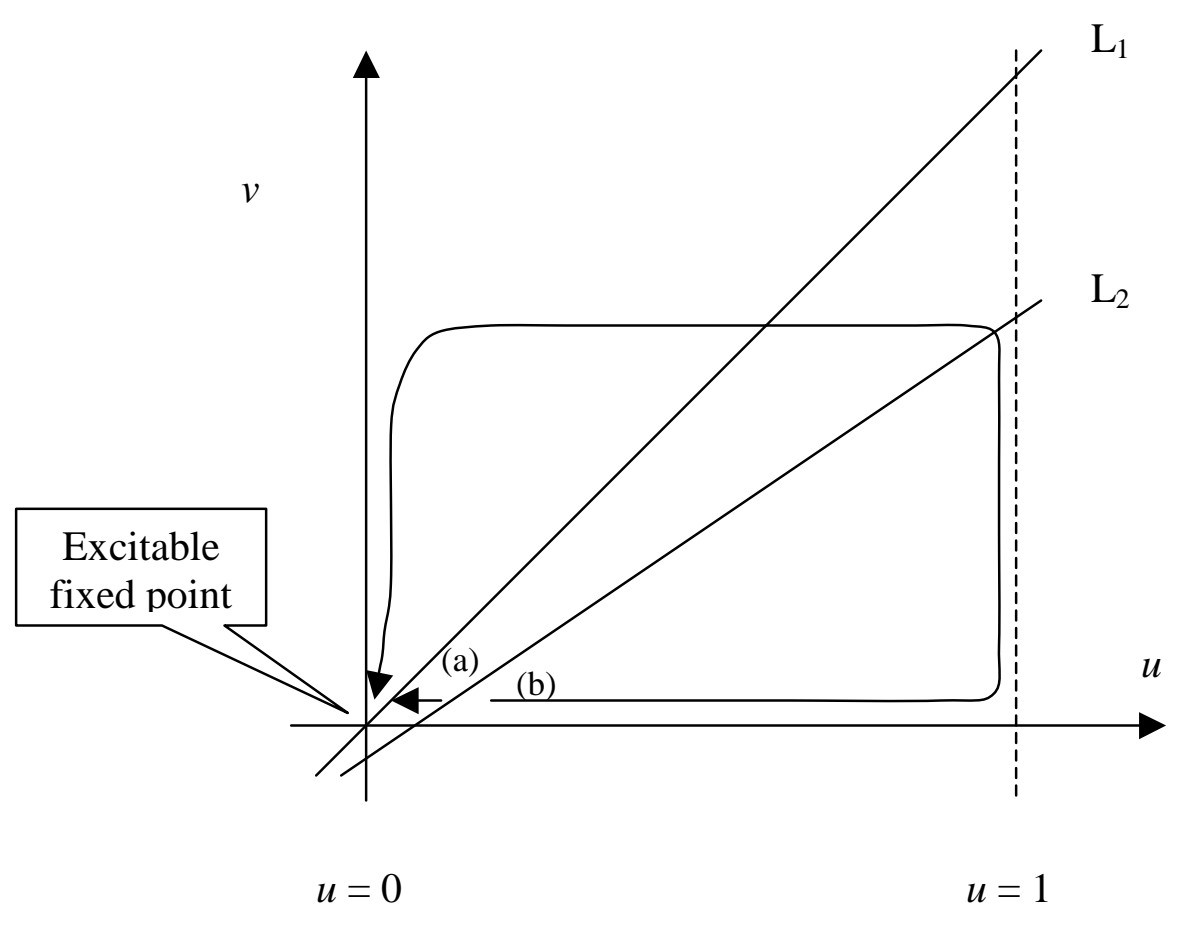

Figure 3.6. Illustration of nullclines in the Barkley model. The nullclines are $u=0, u=1, \mathrm{~L}_{1}: v=u$, and $\mathrm{L}_{2}: v=a u-b(1>a>0, b>0)$. The evolution of a subthreshold (a) and a superthreshold (b) excitation is illustrated. 


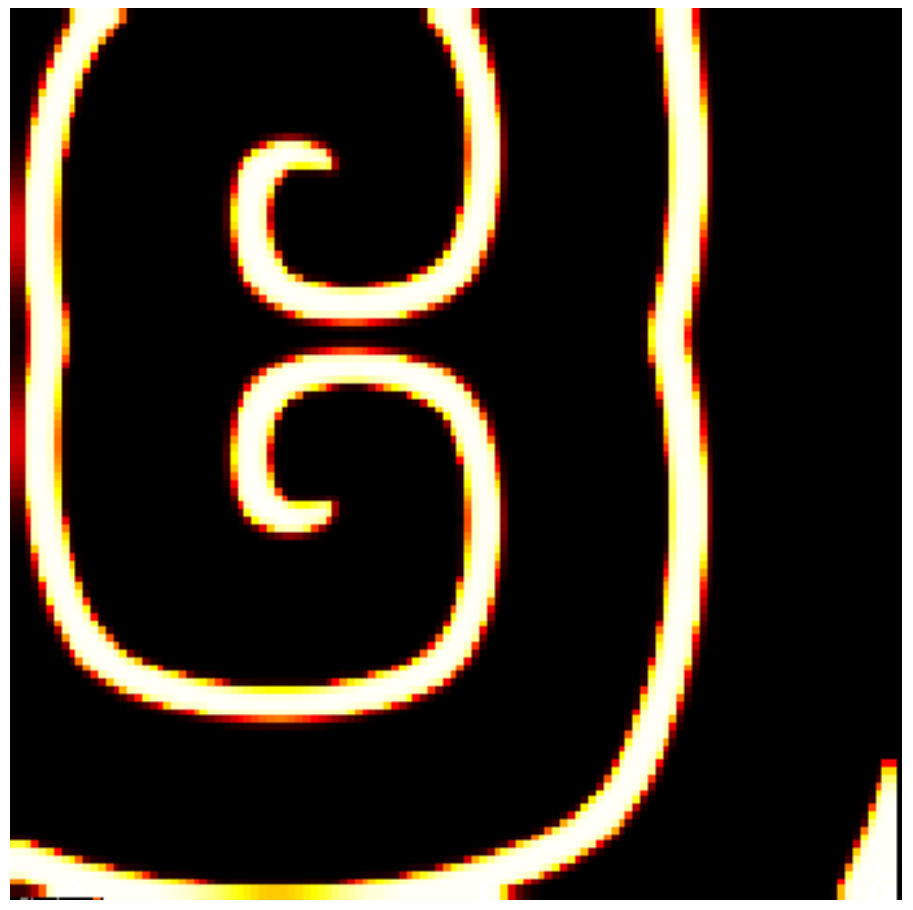

Figure 3.7. A simulation of counterrotating spiral waves with the Barkley model. $a=0.4, b=0.01$, and $\varepsilon=0.005 . L=40, \delta L=$ 0.3 , and $\delta t=0.001$. 


\subsubsection{Pattern synchronization}

We now present a mutual coupling mechanism for synchronizing spatiotemporal behavior by using a modified Barkley model. The coupled system is given by

$$
\begin{aligned}
& \frac{\partial u}{\partial t}=f(u, v, w)+\nabla^{2} u, \\
& \frac{\partial v}{\partial t}=g(u, v, w), \\
& \frac{\partial w}{\partial t}=h(u, v, w)+\nabla^{2} w,
\end{aligned}
$$

where

$$
\begin{aligned}
& f(u, v, w)=\varepsilon^{-1} u(1-u)\left[u-\frac{(v-p(w))+b}{a}\right], \\
& g(u, v, w)=u+w-v, \\
& h(u, v, w)=\varepsilon^{-1} w(1-w)\left[w-\frac{(v-p(u))+b}{a}\right],
\end{aligned}
$$

and $p(w)$ and $p(u)$ can be expanded as polynomials of variables $w$ and $u$, respectively. For simplicity, we take them to be linear, that is, $p(w)=c_{u} w$ and $p(u)=c_{w} u$, where $c_{u}$ and $c_{w}$ are the coupling coefficients. The coupling is also multiplicative because of the existence of the products $u w, u w^{2}$ and $u^{2} w$. This model can have two scenarios: (1) It implies competition between $u$ and $w$ for $v$, and $u$ and $w$ follow similar dynamics but can be differentiated from each other by either physical or chemical methods. (2) If $v$ splits into $v_{1}$ and $v_{2}$ for isolated subsystems $\left(u, v_{1}\right)$ and $\left(w, v_{2}\right)$, the coupling terms can be regarded as perturbations to the media, for example, optical perturbations in a system that is photosensitive. Meanwhile, the perturbation to $u$ (or $w$ ) is simply an image of $w$ (or $u$ ) under a proportional modification. 
It is straightforward to prove the existence of synchronization between $u$ and $w$ provided that $c_{u}=c_{w}$ and, upon the synchronization, the equations can be reduced to a slightly modified version of the Barkley model with the nullclines $u=v$ being replaced by $v=2 u$ and $v=a u-b$ being replaced by $v=\left(a+c_{u}\right) u-b$ as shown on the $u$-v plane (Fig. 3.8). The reduced equations are

$$
\begin{aligned}
& \frac{\partial u}{\partial t}=\varepsilon^{-1} u(1-u)\left[\left(1+\frac{c_{u}}{a}\right) u-\frac{v+b}{a}\right]+\nabla^{2} u, \\
& \frac{\partial v}{\partial t}=2 u-v,
\end{aligned}
$$

plus an algebraic relation $w=u$. The equations describe the common dynamics of $w$ and $u$ upon their synchronization.

In order to examine the stability of the synchronization, we take a variation of Eq. (3.7) on the difference of $d=u-w$ and let $c \equiv c_{u}=c_{w}$. This gives

$$
\frac{\partial d}{\partial t}=-\varepsilon^{-1} \lambda d
$$

where

$$
\lambda=\frac{v+b}{a}-\left(1+\frac{v+b}{a}\right)(u+w)+u^{2}+\left(1+\frac{c}{a}\right) u w+w^{2} .
$$

If $\lambda \geq 0, d \rightarrow 0$, which means that the synchronization is stable. It is otherwise unstable. In general, $\lambda$ depends on the evolution of $u, v$ and $w$, so we need to integrate Eq. (3.10) and (3.11) together with the original Eq. (3.7) and (3.8). However, due to the fast dynamics that causes the system to spend only a very short time during the transition between the states $u$ (or $w$ ) $\sim 0$ and $u$ (or $w$ ) $\sim 1$, we only need to know whether or not the synchronous states $(u, w) \sim(0,0)$ and $(u, w) \sim(1,1)$ are stable. Clearly, for $(u, w) \sim$ $(0,0), \lambda \sim(v+b) / a \geq 0$, since $a, b$, and $v$ are all positive. For $(u, w) \sim(1,1)$, we have 


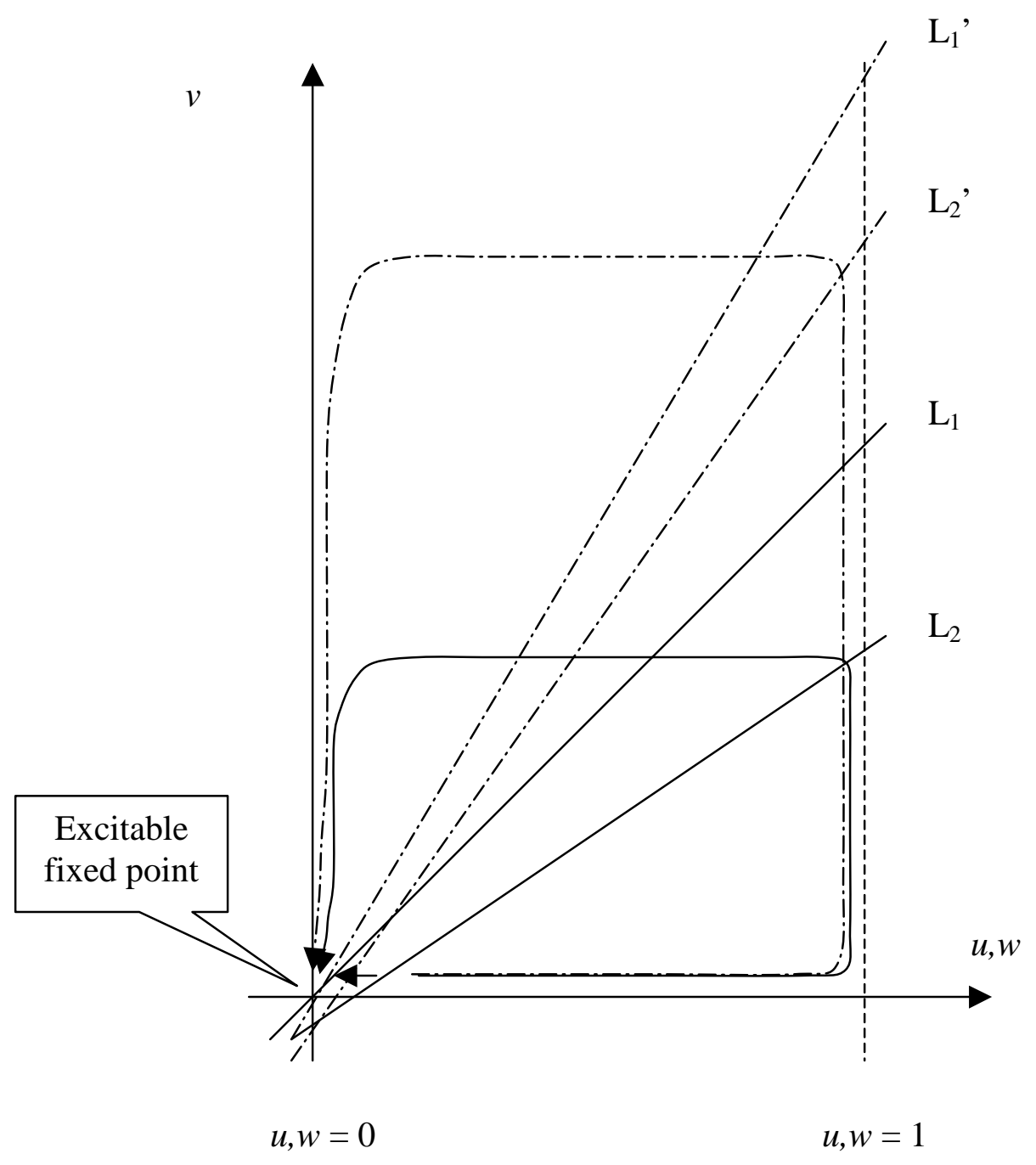

Figure 3.8. Illustration of the nullclines in the coupled Barkley model. The original nullclines are $u=0, u=1, \mathrm{~L}_{1}: v=u$, and $\mathrm{L}_{2}: v=a u-b(1>a>0, b$ $>0)$. For synchronization, $\mathrm{L}_{1}{ }^{\prime}(v=2 u)$ replaces $\mathrm{L}_{1}$ and $\mathrm{L}_{2}{ }^{\prime}(v=(a+c) u-b)$ replaces $\mathrm{L}_{2}$. Upon synchronization, the excursion resulting from the excitation becomes longer than that without synchronization. In the spatiotemporal case, this means the wave front will broaden. 


$$
\lambda \sim 1-\frac{v-c+b}{a}
$$

Compared with the nullcline $v=\left(a+c_{u}\right) u-b$, i.e., $1-(v-c u+b) / a=0$, we know that $1-$ $(v-c+b) / a>0$ before the system turns into the fast-dynamics stage from the state $(u, w) \sim$ $(1,1)$, which can be represented by the trajectory right below the nullcline $v=\left(a+c_{u}\right) u-$ $b$ on the $u, w$ vs. $v$ plane (Fig. 3.8). Therefore, the $u$ and $w$ can be stably synchronized to the two states. The ability to consider only the two states arises from the slow manifold features of the Barkley model. Under other situations and in other models, one must resort to numerical methods to prove the stability of the synchronization.

In Fig. 3.9, we show a numerical integration of the corresponding ODE equations of Eq. (3.7) with the initial conditions of $u, v, w$ chosen with the following values: $u_{0}$ is above the excitation threshold while $w_{0}$ is below the threshold but not zero (the nonzero value can be regarded as an initial seeding or may result from the diffusion of $w$ from the spatial neighborhood). When $w=0$, the local dynamics of the system simply follows the original Barkley model as given by Eq. (3.5). The figure shows the coupling and interaction among the variables can lift $w$ very quickly to the synchronous state at $(u, w)$ $\sim(1,1)$ after it crosses the threshold (note the time scale on the $t$-axis). After the turning point to the fast-dynamics stage, $u$ and $w$ maintain the synchronization in the $(0,0)$ state. In the ODE simulation, $(u, w)=(0,0)$ implies the ending state of the excitation. However, in the PDE simulation of Eq. (3.6), due to diffusion and wave propagation, the local media can be reexcited.

Fig. 3.10 and 3.11 show the 2D simulated behavior of the system. In Fig. 3.10, a $120 \times 120$ lattice $(L=40$ and $\delta L=0.3)$ is used and the Laplacian is approximated by a five-point average. $u$ and $w$ are initialized with high concentrations at different spatial locations as shown by the bright bars in the plot. On one side of the bars, we add an initial layer of the inhibitor $v$ so that the system can evolve into complex patterns, for example, spirals. $u=v=w=0$ anywhere else. Non-flux boundary conditions and an explicit Euler method are used for the integration $(\delta t=0.0002)$. Along with the evolution 


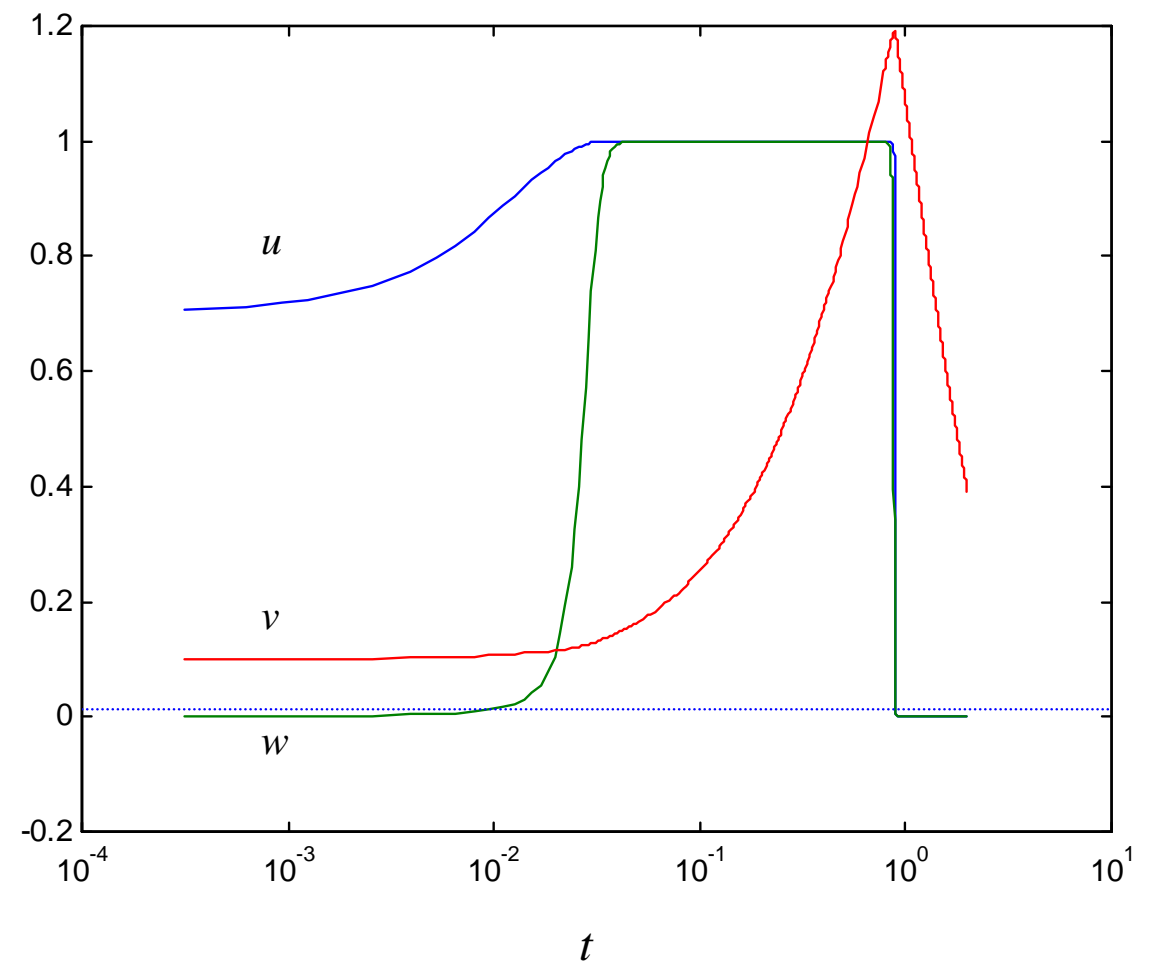

Figure 3.9. An ODE simulation (local dynamics) shows the excitation of $w$ due to coupling. $u$ and $w$ synchronize at states $(u, w)=(1,1)$ and $(0,0) . \varepsilon=$ $0.005, a=0.4, b=0.001$ and $c_{u}=c_{v}=0.6$. 
$t=0$

$t=2500$
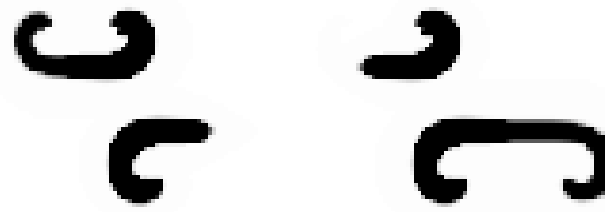

$t=3500$

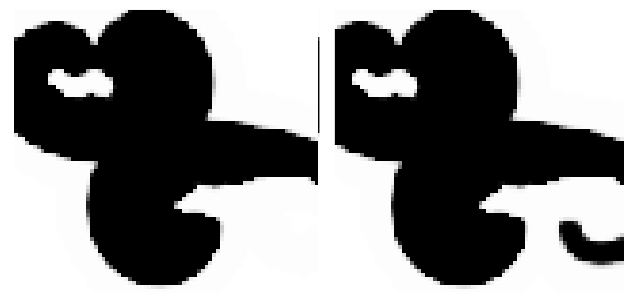

$t=5000$

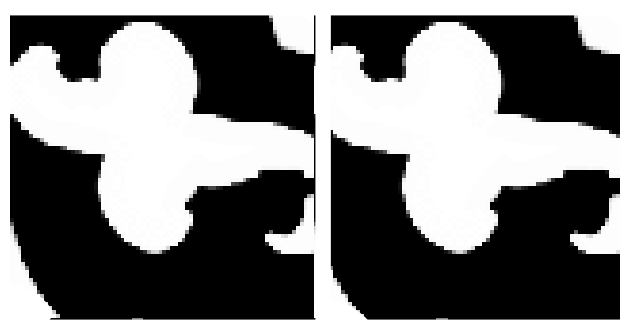

Figure 3.10. 2D simulation of the coupled Barkley model. The left and right panels show the evolution of $u$ and $w$, respectively. The initial perturbations are shown as black bars at $\mathrm{t}=0 . a=0.38, b=0.002$, and $\varepsilon=0.0048 . L=40, \delta L=0.3$, and $\delta t=0.0002$. 


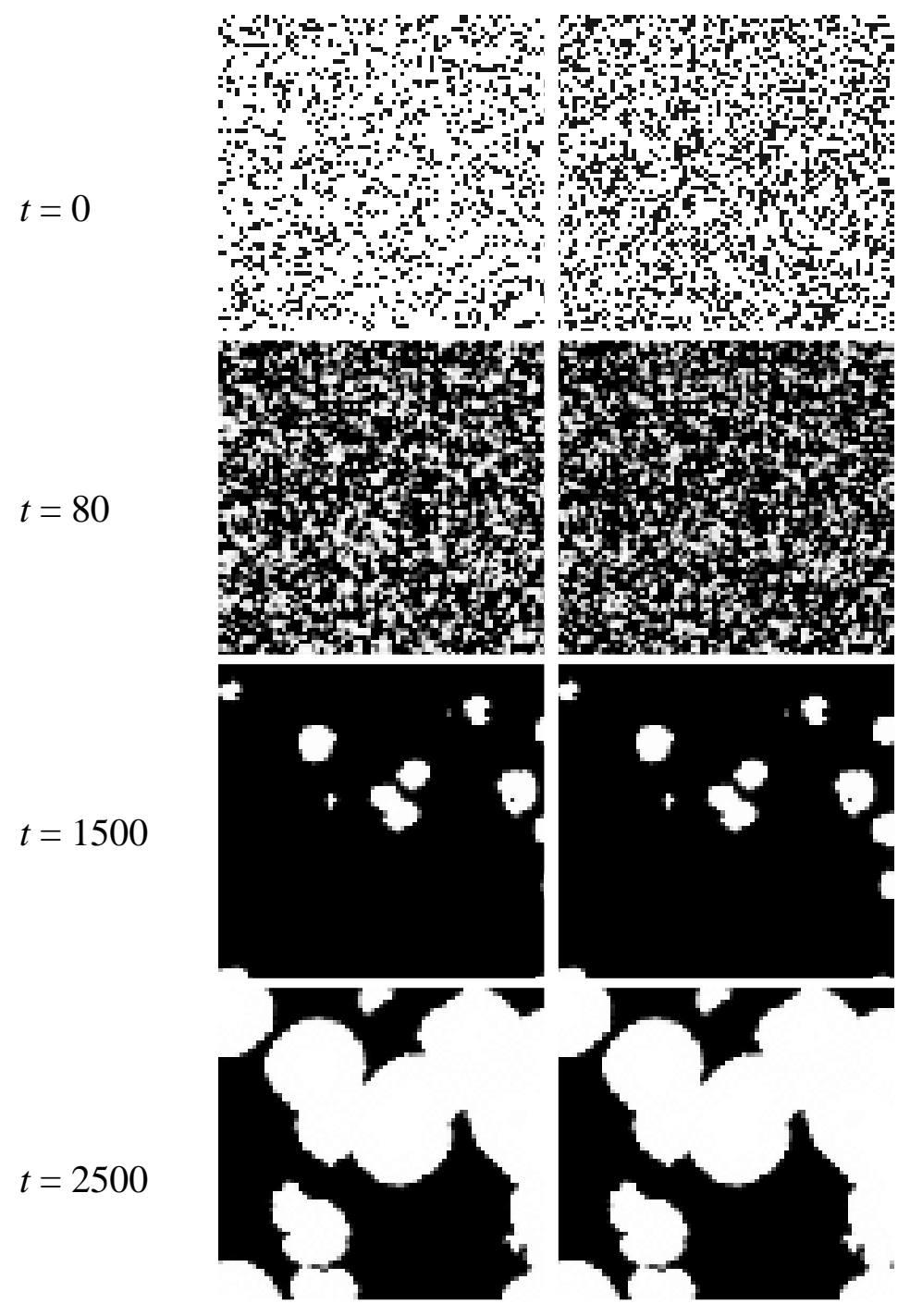

Figure 3.11. 2D simulation of the coupled Barkley model. The left and right panels show the evolution of $u$ and $w$, respectively. The initial perturbations are randomly distributed, with the initial perturbations in $w$ more dense than those in $u$. Parameter conditions are the same as those in Fig. 3.10. 
of the system, both $u$ and $w$ diffuse into the regions where they are initially absent. Before any overlapping of the occupations of $u$ and $w$, the system obeys the original Barkley model. Once the occupations of $u$ and $w$ begin to overlap due to the diffusion and wave propagation, the system follows the dynamics as determined by the coupling model. The more highly concentrated species, for example, $u$, will cause the lower concentrated species, $w$, to be excited with synchronization occurring (see Fig. 3.9). The synchronized wave fronts are apparently broadened compared to the original system, since the duration of the excitation period should be lengthened upon synchronization. Fig. 3.11 shows another example of pattern synchronization from spatially random initializations of $u, v$, and $w$, while the other conditions are unchanged. We note that the synchronous patterns do not last forever because under non-flux boundary condition, the excited waves will disappear when they reach the boundaries. However, synchronization is achieved very quickly before the disappearance of the waves. The lifetime of the synchronized system is closely related to the spatial size of the system.

\subsection{Conclusion}

In this chapter, we have studied chaos synchronization in an ODE and a PDE system, using both mutual and multiplicative coupling schemes simultaneously. In the ODE system, chaos synchronization happens in a way that one subsystem is simply an expansion (or contraction) of another subsystem in the phase space. The expansion ratio, however, depends on initial conditions, similar to the uncertainty in a high-dimensional dynamical system we studied in Chapter 2. In the PDE system, patterns are found to synchronize each other completely. Specially configured initial conditions and randomly distributed initial conditions both lead to complete synchronization. 


\subsection{Appendix: MatLab programs for the simulations}

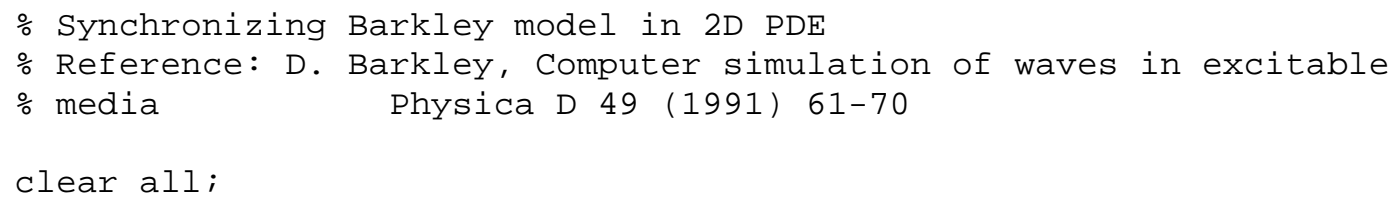




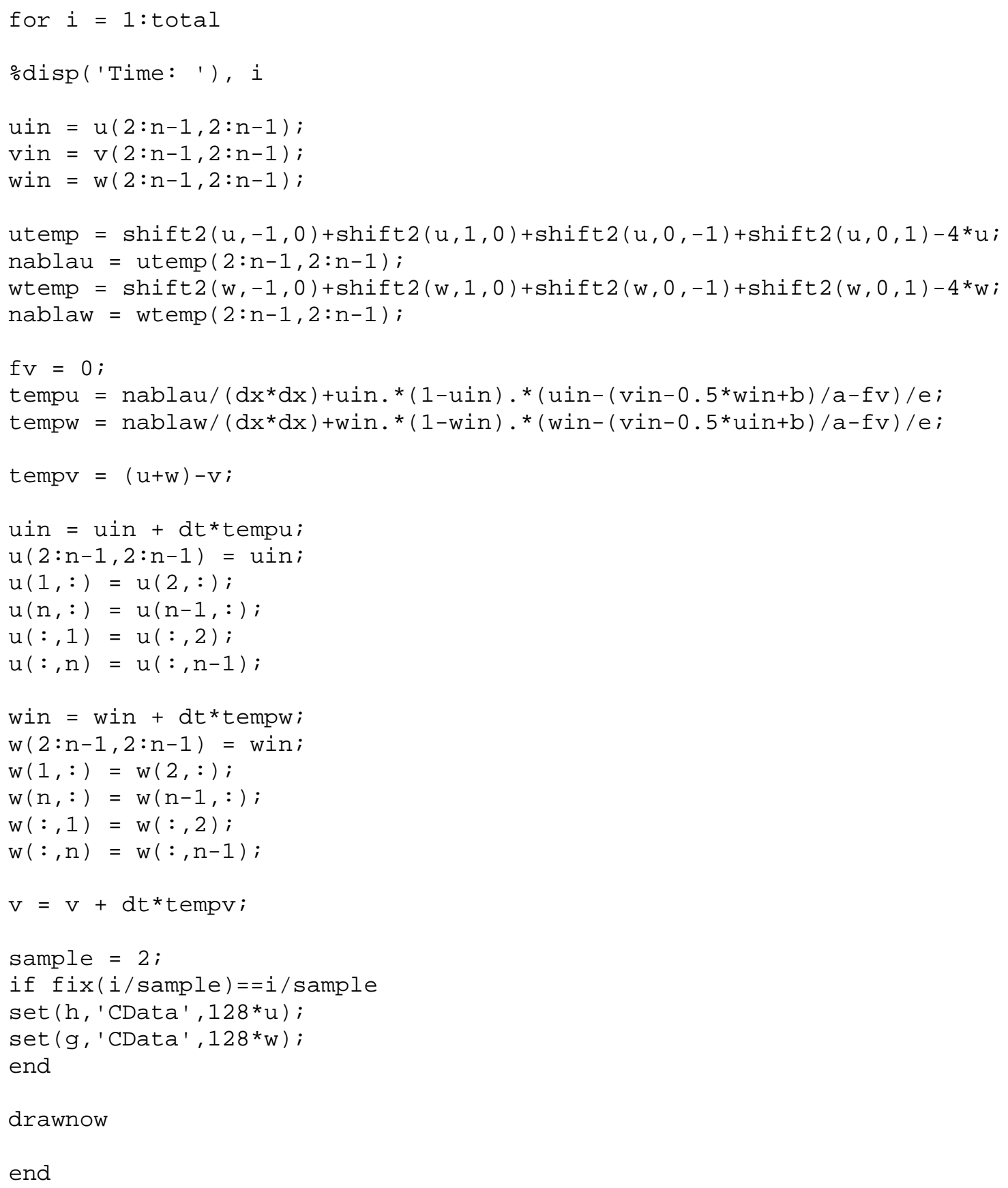




\subsection{References}

1. C. Hugenii, Horoloqium Oscilatorium (Paris, France, 1673).

2. A. Andronov, A. Vitt, and S. Khaykin, Theory of Oscillations (Pergamon, Oxford, 1966).

3. C. Hayashi, Nonlinear Oscillations in Physical Systems (McGraw-Hill, New York, 1964).

4. I. I. Blekhman, Synchronization of Dynamical Systems (Nauka, Moscow, 1971) (in Russian).

5. I. I. Blekhman, Synchronization in Science and Technology (Nauka, Moscow, 1981) (in Russian); English translation: 1988, ASME Press, New York.

6. H. D. I. Abarbanel, R. Brown, J. J. Sidorowich, and L. Sh. Tsimring, Rev. Mod. Phys. 65, $1331(1993)$.

7. Y. Kuramoto, Chemical Oscillations, Waves, and Turbulence (Springer, New York, 1984).

8. H. Fujisaka and T. Yamada, Prog. Theor. Phys. 69, 32 (1983).

9. A. S. Pikovsky, Z. Phys. B 55, 149 (1984).

10. V. S. Afraimovich, N. N. Verichev, and M. I. Rabinovich, Radiophys. Quantum Electron. 29, 795 (1986).

11. L. M. Pecora and T. L. Carroll, Phys. Rev. Lett. 64, 821 (1990).

12. K. M. Cuomo and Z. V. Oppenheim, Phys. Rev. Lett. 71, 65 (1993). 
13. R. Roy, T. W. Murphy, T. D. Maiser, Z. Gills, and E. R. Hunt, Phys. Rev. Lett. 68, 1259 (1992).

14. H. G. Winful and L. Rahman, Phys. Rev. Lett. 65, 1575 (1990).

15. L. Kocarev, Z. Tasev, T. Stojanovski, and U. Parlitz, Chaos 7, 635 (1997).

16. Y. Braiman, J. F. Lindner, and W. L. Ditto, Nature 378, 465 (1995).

17. S. Boccaletti, J. Bragard, F. T. Arecchi, and H. Mancini, Phys. Rev. Lett. 83, 536 (1999).

18. F. S. de San Roman, S. Boccaletti, D. Maza, and H. Mancini, Phys. Rev. Lett. 81, 3639 (1998).

19. D. H. Zanette and A. S. Mikhailov, Phys. Lett. A 235, 135 (1997).

20. J. H. Peng, E. J. Ding, M. Ding, and W. Yang, Phys. Rev. Lett. 76, 904 (1996).

21. M. Ding, E. J. Ding, W. L. Ditto, B. Gluckman, V. In, J. H. Peng, M. L. Spano, and W. Yang, Chaos 7, 644 (1997).

22. M. G. Rosenblum, A. S. Pikovsky, and J. Kurths, Phys. Rev. Lett. 76, 1840 (1996).

23. M. G. Rosenblum, A. S. Pikovsky, and J. Kurths, Phys. Rev. Lett. 78, 4193 (1997).

24. L. Kocarev and U. Parlitz, Phys. Rev. Lett. 76, 1816 (1996).

25. K. Pyragas, Phys. Lett. A 170, 421 (1992).

26. T. C. Newell, P. M. Alsing, A. Gavrielides, and V. Kovanis, Phys. Rev. Lett. 72, 1647 (1994).

27. S. Boccaletti, A. Farini, and F. T. Arecchi, Phys. Rev. E 55, 4979 (1997). 
28. V. S. Anishchenko, T. E. Vadivasova, D. E. Posnov, N. N. Verichev, and M. I. Rabinovich, Sov. J. Commun. Technol. Electron. 36, 23 (1991).

29. A. Maritan, J. R. Banavar, Phys. Rev. Lett. 72, 1451 (1994).

30. A. V. Gaponov-Grekhov, M. I. Rabinovich, and I. M. Starobinets, Pis'ma v Zh. Eksp. Teor. Fiz. 39, 561 (1984).

31. H. Sun, S. K. Scott, and K. Showalter, Phys. Rev. E 60, 3876 (1999).

32. M. C. Cross and P. C. Hohenberg, Rev. Mod. Phys. 65, 851 (1993).

33. A. S. Mikhailov and I. V. Uporov, Sov. Phys. Usp. 27, 695 (1984).

34. V. V. Barelko, in Self-organization, Autowaves and Structures Far from Equilibrium, edited by V. I. Krinsky, p. 164, (Springer-Verlag, Berlin, 1984).

35. R. J. Field and M. Burger, Oscillations and Travelling Waves in Chemical Systems (Wiley, New York, 1985).

36. A. T. Winfree, When Time Breaks Down (Princeton University Press, Princeton, 1987).

37. R. J. Field, E. Koros, and R. M. Noyes, J. Am. Chem. Soc. 94, 8649 (1972).

38. J. J. Tyson and P. C. Fife, J. Chem. Phys. 73, 2224 (1980).

39. D. Barkley, Physica D 49, 61 (1991).

40. D. Barkley, Phys. Rev. Lett. 68, 2090 (1992).

41. S. H. Strogatz, Nonlinear Dynamics and Chaos (Addison-Wesley, New York, 1994). 


\section{Chapter 4}

\section{Self-Segregation of Competitive Chaotic Populations}

\subsection{Introduction}

In the first two chapters, we have studied uncertainty and synchronization in chaotic systems and have focused on the mechanisms and techniques for generating or reducing complexity in a dynamical system. In the following two chapters, the complexity of pattern formation is studied in reaction-diffusion systems, where competition and cooperation among species play significant roles. These studies are relevant to physics, chemistry and to population biology. ${ }^{1-5}$

In natural and social environments, we can notice the interplay of competition and cooperation. Competition has two main characteristics: 1) two or more species compete for a common resource, and 2) one species depends on another species, which is characterized by a predator-prey relationship. Cooperation, on the other hand, typically

embodies characteristics of altruism, which facilitates the coexistence of species. ${ }^{6}$ Competition may lead to the segregation of species into different spatial domains, while cooperation between species may result in an integration of populations.

In the following chapter, we discuss pattern formation in competitive environments, which has been the subject of numerous studies in physics, ${ }^{7-9}$ chemistry, ${ }^{10,11}$ ecology, ${ }^{12}$ and genetics. ${ }^{13}$ Non-classical phenomena, such as spontaneous cluster formation, reactant segregation, and depletion-zone formation, have been investigated in diffusion-controlled chemical reactions. ${ }^{10}$ Another class of systems, in which two populations compete for a 
common sustaining resource, has yielded the ecologically motivated and controversially discussed "competitive exclusion principle", ${ }^{12}$ where two species with similar characteristics are unable to coexist. In this context, closed reaction-diffusion systems exhibit exclusive product selectivity when the species have different diffusivities or different rates of autocatalysis, ${ }^{11}$ or in the case of open Lotka-Volterra kinetics (with equal diffusivities), segregation of steady-state populations. ${ }^{13}$ Segregation, as the term suggests, precludes the coexistence of two or more species in a spatial domain, that is, the survival of one species in a domain incurs the vanishing of another species in the same domain, despite nonzero initial concentrations for both species.

The dynamics of segregation has been an active research topic in a broad spectrum of research areas. ${ }^{14-16}$ The studies range from identifying dynamical premises to analyzing the segregation structure and studying the motion of domain boundaries or interfaces. However, segregation of species that exhibit chaotic dynamics within each domain has not been reported, although it might be common in nature. Here, we present a reaction-diffusion system with chaotic dynamics that exhibits complex interface patterns arising from self-segregation behavior. ${ }^{14} \mathrm{We}$ study the evolution of coupled reactions, each governed by cubic autocatalysis, ${ }^{17}$ which compete for a common resource. ${ }^{18,19}$ Selfsegregating domains of uncorrelated chaotic populations, separated by interfaces that exhibit irregular motions on long time scales, spontaneously arise in one-dimensional configurations with random initial conditions. Localized interfaces are exhibited for steady state populations or for correlated chaotic populations arising from symmetric initial conditions. Curvature-induced interface drift governs the pattern evolution in twodimensional configurations, which develops on a time scale much longer than that of the population dynamics and yields complex reorganizations at interface junctions in the case of three or more autocatalytic species.

This chapter is organized as follows: In Section 4.2, we review the relevant features of the Gray-Scott model, a two-variable reaction-diffusion system, which generates chaotic population dynamics for a certain parameter range. On the basis of this model, we construct a competitive reaction-diffusion system and present an analysis of the dynamic 
features. We present numerical results and discuss the novel dynamic features of the system in Section 4.4. We draw our conclusions in Section 4.5.

\subsection{Illustration of Reaction-Diffusion without Competition}

In 1984, Gray and Scott ${ }^{17}$ proposed a reaction-diffusion model with cubic autocatalysis, which has been extensively studied in the past years. This model exhibits characteristic features of excitability under conditions of an open reactor. For example, it can show the existence of wave reflection and spontaneous wave splitting in one spatial dimension, depending on the relative diffusivities of the variable species. In 1996, Merkin et al. ${ }^{20-22}$ further showed that the model exhibits spatiotemporal chaos when propagating waves interact with local instabilities. Our study is based on an extension of this model.

The Gray-Scott model is comprised of an autocatalytic reaction,

$$
A+2 B \rightarrow 3 B \quad\left(\text { rate }=k_{1} a b^{2}\right)
$$

which is coupled with a single decay step

$$
B \rightarrow C \quad\left(\text { rate }=k_{2} b\right)
$$

where $a$ and $b$ are the concentrations of the reactant $A$ and autocatalyst $B$, respectively. This reaction is arranged in a continuous-flow unstirred reactor (CFUR) that allows a continuous supply of fresh reactants in a way that does not disrupt the transport process of molecular diffusion. A schematic representation for this model is given in Fig. 4.1. The reaction zone is in contact with reservoirs from which fresh reactants can diffuse through a permeable membrane into the reaction zone. In addition, reaction products can be removed from the reaction zone through this membrane. 


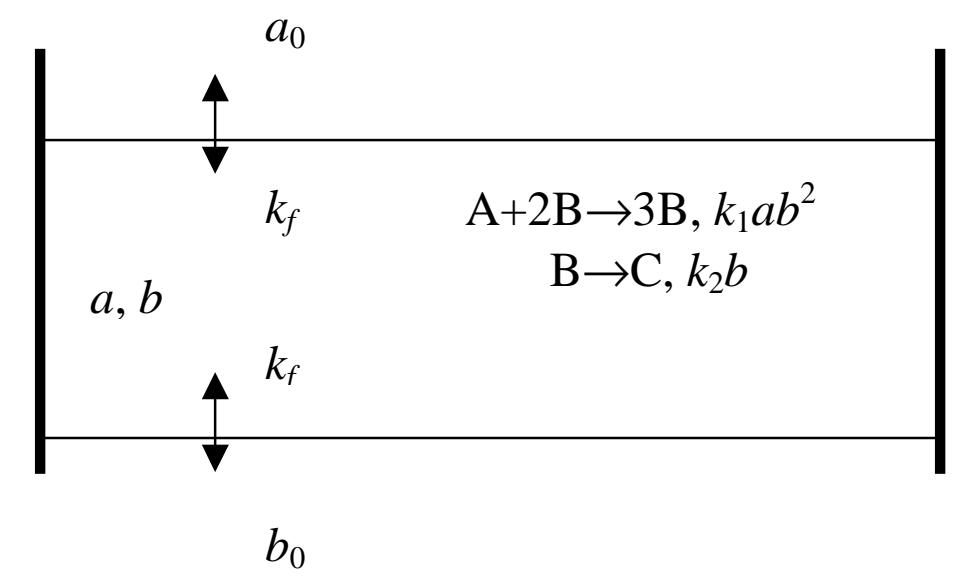

0

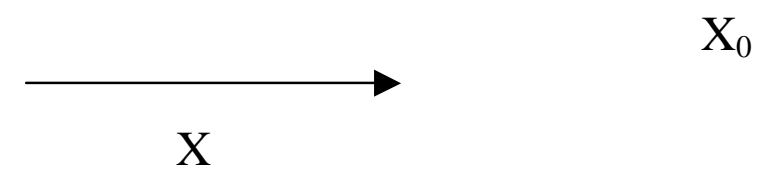

Figure 4.1. Schematic representation of the CFUR system (Merkin et al., J. Chem. Soc. Faraday Trans. 92, 2911 (1996)).

Suppose that in the reservoirs reactant $A$ and species $B$ are maintained at some constant concentration $a_{0}$ and $b_{0}$, respectively. In this case, we can write the following reaction-diffusion equations: ${ }^{17,21}$

$$
\begin{aligned}
& \frac{\partial a}{\partial t}=D_{A} \nabla^{2} a+k_{f}\left(a_{0}-a\right)-k_{1} a b^{2} \\
& \frac{\partial b}{\partial t}=D_{B} \nabla^{2} b+k_{f}\left(b_{0}-b\right)+k_{1} a b^{2}-k_{2} b
\end{aligned}
$$

For a spatially one-dimensional system, these equations can be transformed into a dimensionless form by scaling the concentrations as $\alpha=a / a_{0}, \beta=b / a_{0}$ and $\beta_{0}=b_{0} / a_{0}$, time as $\tau=t / t_{e x}$ where $t_{e \mathrm{x}}=1 / k_{f}$, and length as $x=X / L$ where $L=\sqrt{ }\left(D_{\mathrm{B}} t_{e x}\right)$. This scaling leads to:

$$
\begin{aligned}
& \frac{\partial \alpha}{\partial \tau}=\delta \nabla^{2} \alpha+1-\alpha-\mu \alpha \beta^{2} \\
& \frac{\partial \beta}{\partial \tau}=\nabla^{2} \beta+\beta_{0}-\phi \beta+\mu \alpha \beta^{2}
\end{aligned}
$$


with $\mu=k_{1} a_{0}^{2} / k_{f}$ and $\phi=\left(k_{f}+k_{2}\right) / k_{f}$. The parameter $\delta=D_{\mathrm{A}} / D_{\mathrm{B}}$ is the ratio of the diffusion coefficients.

\subsubsection{Stability Analysis}

To analyze the stability of system (4.4), we first identify its steady states in the spatially homogenous case, where $\nabla^{2} \alpha=\nabla^{2} \beta=0$. The local stability of the steady states is directly relevant to the behavior observed in the full model. Further, we focus on the situation where $\beta_{0}=0$. This approximation only shifts the locations of the steady states in phase space and does not affect the stability analysis. With the above settings, we obtain the steady states by solving the following set of algebraic equations

$$
\begin{aligned}
& 1-\alpha-\mu \alpha \beta^{2}=0 \\
& -\phi \beta+\mu \alpha \beta^{2}=0
\end{aligned}
$$

There are three solutions: the "extinguished" steady state $\left(\alpha_{\mathrm{ss}}, \beta_{\mathrm{ss}}\right)=(1,0)$ and a pair of symmetric solutions

$$
\left(\alpha_{s s}{ }^{ \pm}, \beta_{s s}{ }^{ \pm}\right)=\left(\frac{\mu \mp \sqrt{\mu^{2}-4 \mu \phi^{2}}}{2 \mu}, \frac{\mu \pm \sqrt{\mu^{2}-4 \mu \phi^{2}}}{2 \mu \phi}\right) .
$$

Hence, there is a locus in the $\mu$ vs $\phi$ plane (Fig. 4.2), which is determined by

$$
\mu_{s n}=4 \phi^{2}
$$

and $\left(\alpha_{\mathrm{ss}}{ }^{ \pm}, \beta_{\mathrm{ss}}{ }^{ \pm}\right)$only exist for $\mu \geq \mu_{\mathrm{sn}}$.

Next, in order to see the stability of the above steady states, we perform a linear functional analysis on Eq. (4.4) as follows: 


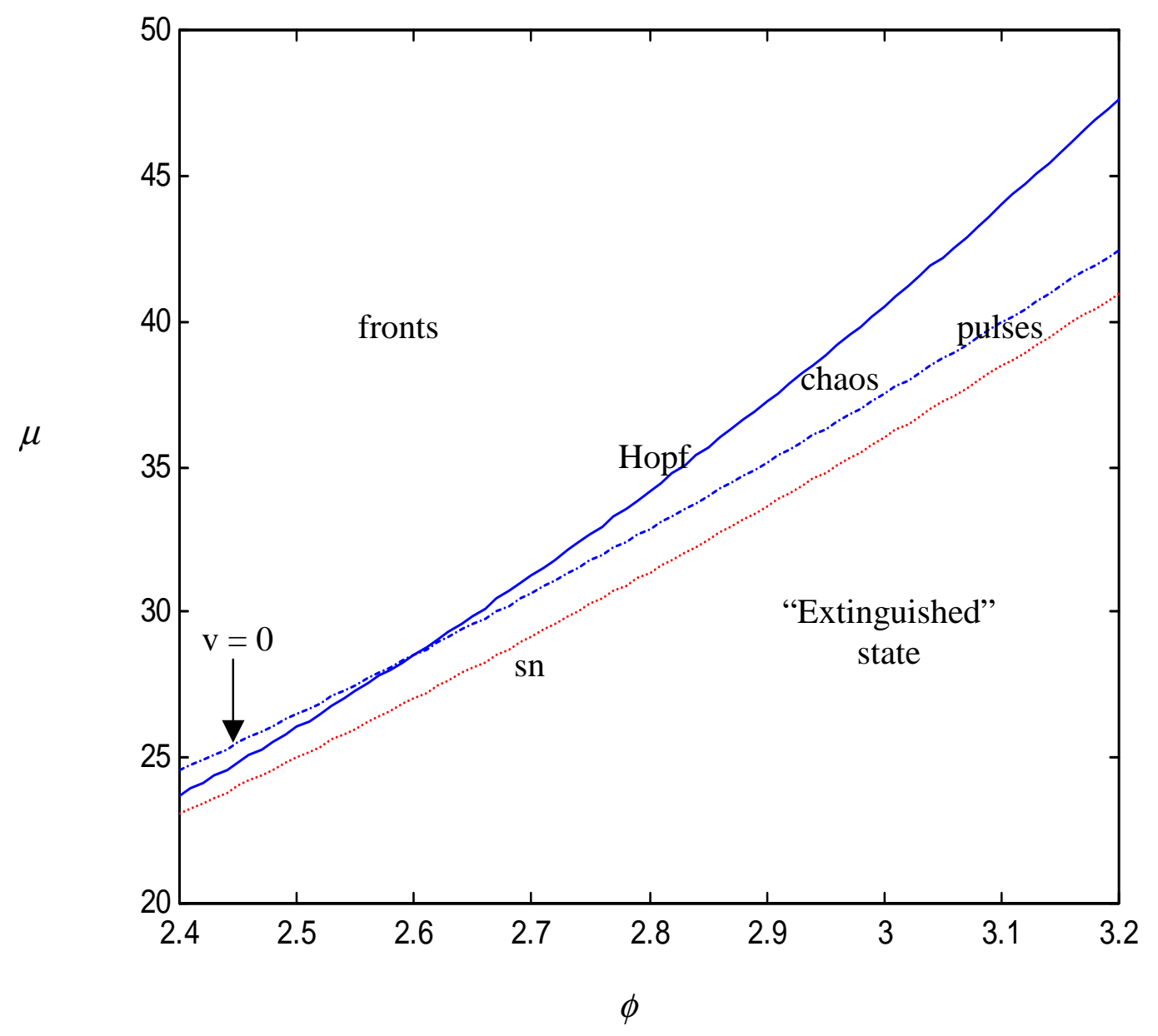

Figure 4.2. Bifurcation diagram showing the dependence of the Hopf bifurcation and saddle-node bifurcation on the parameter $\phi$ and $\mu$ as given by Eqs. (4.7) and (4.11). The curve on which the front velocity, $v=0$, is shown, as are the regions of parameter space in which fronts and pulses are obtained. The region of chaotic behavior is indicated. (Merkin et al., Phys. Rev. Lett. 76, 546 (1996)). 


$$
\frac{\partial}{\partial t}\left(\begin{array}{l}
\delta \alpha \\
\delta \beta
\end{array}\right)=\left(\begin{array}{cc}
-1-\mu \beta^{2} & -2 \mu \alpha \beta \\
\mu \beta^{2} & -\phi+2 \mu \alpha \beta
\end{array}\right)\left(\begin{array}{l}
\delta \alpha \\
\delta \beta
\end{array}\right)
$$

where $\delta \alpha$ and $\delta \beta$ are variations on $\alpha$ and $\beta$, respectively. Then, the Jacobian matrix associated with the steady states reads,

$$
J_{s s}=\left(\begin{array}{cc}
-1-\mu \beta^{2} & -2 \mu \alpha \beta \\
\mu \beta^{2} & -\phi+2 \mu \alpha \beta_{s s}
\end{array}\right)
$$

For the "extinguished" steady state, $\left(\alpha_{\mathrm{ss}}, \beta_{\mathrm{ss}}\right)=(1,0)$, we have two negative eigenvalues $\lambda_{1}=-1$ and $\lambda_{2}=-\phi(\phi>0)$, which means that the steady state is globally stable on the parameter plane. After some algebra, we obtain the eigenvalues for the steady states $\left(\alpha_{\mathrm{ss}}{ }^{ \pm}\right.$, $\left.\beta_{\mathrm{ss}}{ }^{ \pm}\right)$

$$
\lambda_{ \pm}=\frac{\phi-(\gamma+1) \pm \sqrt{[\phi-(\gamma+1)]^{2}-4(\gamma-1)}}{2}
$$

with $\gamma \equiv \mu\left(\beta_{\mathrm{ss}}{ }^{ \pm}\right)^{2}=\left(1-\alpha_{\mathrm{ss}}{ }^{ \pm}\right) / \alpha_{\mathrm{ss}}{ }^{ \pm}$. For the lower root $\left(\alpha_{\mathrm{ss}}{ }^{-}, \beta_{\mathrm{ss}}{ }^{-}\right)$, we get $\gamma<1$, and thus a pair of real eigenvalues with opposite signs. This shows $\left(\alpha_{\mathrm{ss}}{ }^{-}, \beta_{\mathrm{ss}}{ }^{-}\right)$represents a saddle point. For the upper root $\left(\alpha_{\mathrm{ss}}{ }^{+}, \beta_{\mathrm{ss}}{ }^{+}\right)$, we find $\gamma>1$. Depending on whether $\phi-(\gamma+1)$ is larger than or less than zero, the real parts of both eigenvalues are either positive or negative. Thus, we get a second locus in the $\mu$ vs $\phi$ plane, which corresponds to a Hopf bifurcation and is determined by $\phi-(\gamma+1)=0$. When $\phi>2$, this equation gives

$$
\mu_{H}=\frac{\phi^{4}}{\phi-1}
$$

When $\mu>\mu_{\mathrm{H}},\left(\alpha_{\mathrm{ss}}{ }^{+}, \beta_{\mathrm{ss}}{ }^{+}\right)$is a stable focus; otherwise, it is an unstable focus. (In fact, depending on whether $[\phi-(\gamma+1)]^{2}-4(\gamma-1)>0$, we can further discern a node $\left(\operatorname{Im}\left(\lambda_{ \pm}\right)=0\right)$ from a focus $\left(\operatorname{Im}\left(\lambda_{ \pm}\right) \neq 0\right)$, but the corresponding asymptotic states are equivalent.) The 
Hopf bifurcation emerges from a double-zero eigenvalue point at $\mu=16$ and $\phi=2$, where both $\phi-(\gamma+1)=0$ and $[\phi-(\gamma+1)]^{2}-4(\gamma-1)=0$. The two loci intersect at this emerging point. In summary, the first locus, where a saddle-node bifurcation occurs, gives the critical condition for the existence of the steady states $\left(\alpha_{\mathrm{ss}}{ }^{ \pm}, \beta_{\mathrm{ss}}{ }^{ \pm}\right)$; the second locus, where a Hopf bifurcation occurs, gives the critical condition for the stability of the steady state $\left(\alpha_{\mathrm{ss}}{ }^{+}, \beta_{\mathrm{ss}}{ }^{+}\right)$. Furthermore, by analyzing the periodic solution and its stability for the ODE in eq. (4.4), Merkin et al. ${ }^{21}$ derived a detailed picture about the Hopf bifurcation: For $2<\phi<4$, the Hopf bifurcation is subcritical; an unstable limit cycle emerges when $\mu>\mu_{\mathrm{H}}$. For $\phi>4$, a stable limit cycle emerges when $\mu>\mu_{\mathrm{H}}$.

\subsubsection{Traveling Waves}

Besides the analysis of the local dynamics of system (4.3), a detailed analysis of traveling waves in the system has been carried out by Merkin and Needham. ${ }^{20-22}$ They showed that there are two types of constant form wave solutions. One is the front that takes the system from its initial $(1,0)$ state to the $\left(\alpha_{\mathrm{ss}}{ }^{+}, \beta_{\mathrm{ss}}{ }^{+}\right)$state (Fig. 4.3(a)). Another is the pulse for which the system is in the $(1,0)$ state both ahead and behind the propagating reactiondiffusion event (Fig. 4.3(b)). When $\mu>\mu_{\mathrm{sn}}$ such that the $\left(\alpha_{\mathrm{ss}}{ }^{+}, \beta_{\mathrm{ss}}{ }^{+}\right)$state exists and is not affected by the local stability of this state, the front connection exists for all parameter values. In Fig. 4.2, we show the zero-velocity locus for the front. Only above this locus, where the front velocity is positive, may we expect the front-type structure. Below this locus, we observe the pulse as the long-time behavior. This continues to be true until $\mu$ is decreased to $\mu=\mu_{\mathrm{sn}}$, after which no traveling waves exist and the system always tends to the $(1,0)$ state. ${ }^{22}$ Therefore, in order to achieve spatiotemporal chaos (Fig. 4.3(c)), the parameter values need to be chosen near or above the zero-velocity locus. In this chapter, we study the phenomenon of chaotic segregation. 

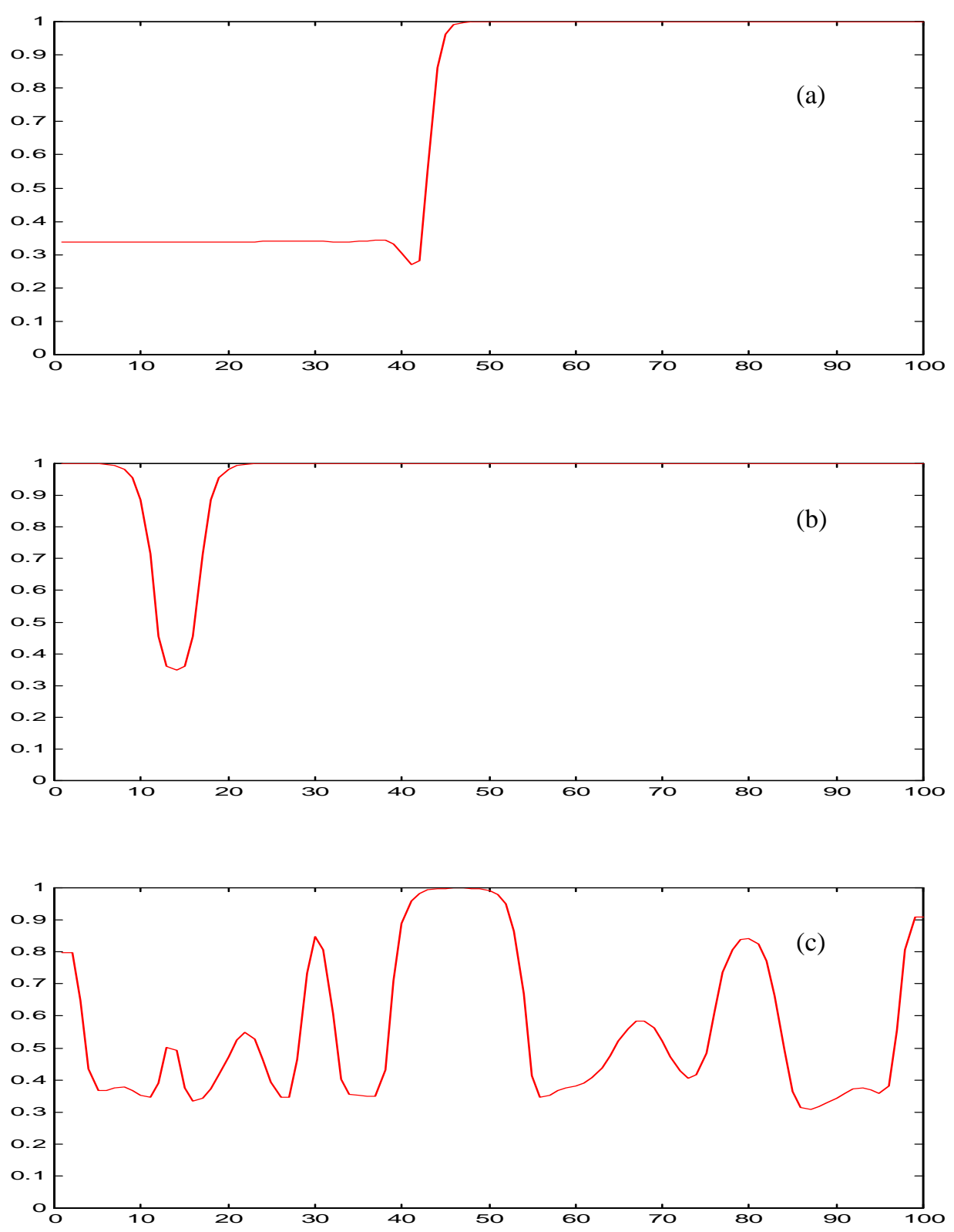

Figure 4.3. Typical instantaneous plots of the $\alpha$-profiles for (a) a traveling front, with $\phi=2.8, \mu=35$; (b) a pulse, with $\phi=2.8, \mu=$ 32.8 and (c) a chaotic response, with $\phi=2.8, \mu=33.15$. (Merkin et al., J. Chem. Soc. Faraday Trans. 92, 2911 (1996)). 


\subsection{The Autocatalator Model with Competition}

Following the review of the two-variable model in Eq. (4.4), we now examine the dynamics of a three-variable model with two autocatalytic species, $B$ and $C$, which compete for a common sustaining resource, $A$, and then decay to products, $P$ and $Q$ :

$$
\begin{array}{ll}
A+2 B \rightarrow 3 B & \left(\text { rate }=k_{1} a b^{2}\right), \\
A+2 C \rightarrow 3 C & \left(\text { rate }=k_{2} a c^{2}\right), \\
B \rightarrow P & \left(\text { rate }=k_{\mathrm{p}} a b^{2}\right), \\
C \rightarrow Q & \left(\text { rate }=k_{\mathrm{q}} a b^{2}\right) .
\end{array}
$$

Dimensionless reaction-diffusion equations for the system in an open spatial reactor are derived following the previous studies described in Section 4.2. The equations read,

$$
\begin{aligned}
& \frac{\partial \alpha}{\partial \tau}=\delta \nabla^{2} \alpha+1-\alpha-\mu \alpha\left(\beta^{2}+\gamma^{2}\right) \\
& \frac{\partial \beta}{\partial \tau}=\nabla^{2} \beta+\beta_{0}-\phi \beta+\mu \alpha \beta^{2} \\
& \frac{\partial \gamma}{\partial \tau}=\nabla^{2} \gamma+\gamma_{0}-\phi \gamma+\mu \alpha \gamma^{2}
\end{aligned}
$$

where $\alpha, \beta$, and $\gamma$ represent the dimensionless concentrations, and $\phi$ and $\mu$ are bifurcation parameters of the system, determined by the flow rate and concentrations of the species in the reservoir (see Section 4.2). Throughout this study, we assume equal diffusivities, $\delta$ $=D_{A} / D_{B}=D_{A} / D_{C}=1$, where $D_{i}$ represents the scaled diffusivity of species $i$, and equal growth rates for autocatalysis in $B$ and $C$, in order to focus on behavior arising from competitive species with similar characteristics. For simplicity, we also assume there is no supply of the autocatalysts from the reservoir, $\beta_{0}=\gamma_{0}=0$. 
Within the parameter range of interest, where the dynamics of the single autocatalyst system (either $\beta \equiv 0$ or $\gamma \equiv 0$ ) in Eqs. (4.17)) exhibits chaotic behavior as discussed before, the coupled system is characterized by the steady states:

$$
S^{\mathrm{A}}=(1,0,0) ; S_{ \pm}^{\mathrm{B}}=\left(a_{ \pm}, h_{ \pm}, 0\right) ; S_{ \pm}^{\mathrm{C}}=\left(a_{ \pm}, 0, h_{ \pm}\right)
$$

where $a_{ \pm}$is equivalent to $\alpha_{\mathrm{ss}}{ }^{\mathrm{r}}$ in Eq. (4.6) with

$$
\begin{aligned}
& a_{ \pm}=\left(1 \pm \sqrt{1-4 \phi^{2} / \mu}\right) / 2 \\
& h_{ \pm}=\phi /\left(\mu a_{ \pm}\right)
\end{aligned}
$$

$S_{ \pm}^{\text {B,C }}$ only exist for $\mu$ above the saddle node bifurcation as denoted by Eq. (4.7). (Another pair of steady states, arising for $\mu>8 \phi^{2}$, are exhibited but are not relevant to this study.) As stated in Section $4.2, S^{\mathrm{A}}$ is a stable node for all parameter values, $S_{+}{ }^{\mathrm{B}, \mathrm{C}}$ are saddle points, and $S_{-}^{\mathrm{B}, \mathrm{C}}$ are unstable foci that become stable for $\mu>\mu_{\mathrm{H}}$ determined by Eq. (4.11). Compared to the single autocatalyst system, we note that the additional, third eigenvalue for each steady state is $-\phi$. The particular symmetry and stability of these states imply that the system is characterized by either an extinct state, $S^{\mathrm{A}}$, or an exclusive state, $S_{ \pm}^{\mathrm{B}, \mathrm{C}}$, where one competing species survives and the other becomes extinct. From the analysis of the bifurcation structure of the single-autocatalyst system shown in Fig. 4.2, it follows that a traveling wave solution with a positive velocity exists only for $\mu$ above a critical value, $\mu_{c} \sim 33$ for $\phi=2.8$, which restricts the parameter space of interest to $\left[\mu_{c} \mu_{\mathrm{H}}\right]$ in the current study. 


\subsection{Numerical Simulations and Analyses}

Numerical simulations are performed on one-dimensional (1D) and two-dimensional (2D) grids. In the 1D simulations, a 3-point approximation of the Laplacian operator and an explicit Euler method are adopted. In the 2D simulations, a 5-point approximation of the Laplacian operator is used.

\subsubsection{Self-segregation in One-dimensional Media}

The spatiotemporal evolution of Eq. (4.17) is shown in Fig. 4.4. After a brief transient period, spatially localized domains develop from randomly distributed perturbations initiating autocatalysis in $B$ and $C$. Species B is restricted to domains where species $C$ is extinct, while species $C$ exists only in domains where species $B$ is extinct. The relative location of the interfaces between the domains is robust with respect to changes in the parameters, as long as traveling wave solutions exist and both autocatalystic species have the same growth rates and diffusivities. The particular location of the interfaces, however, is a consequence of the initial perturbation distribution. The chaotic populations in each of the domains are uncorrelated except for symmetries between domains. The local perturbations must exceed a concentration threshold to initiate autocatalyst, as well as cover a sufficiently large region (Fig. 4.5). Once autocatalysis is initiated, the local trajectory approaches the extinct state, $S^{\mathrm{A}}$. Such transient activity appears in the spatiotemporal pattern as triangular white zones below the initial seeds, where the reactant concentration $\alpha=1$. Waves propagating with constant velocity into the nonreaction zones give rise to domain interfaces when two different autocatalystic species collide. There is, however, a minimum domain size below which a species cannot survive, which depends on the particular parameter values. With these considerations, it is possible to predict the location of the segregation interfaces for different known initial seed configurations. 


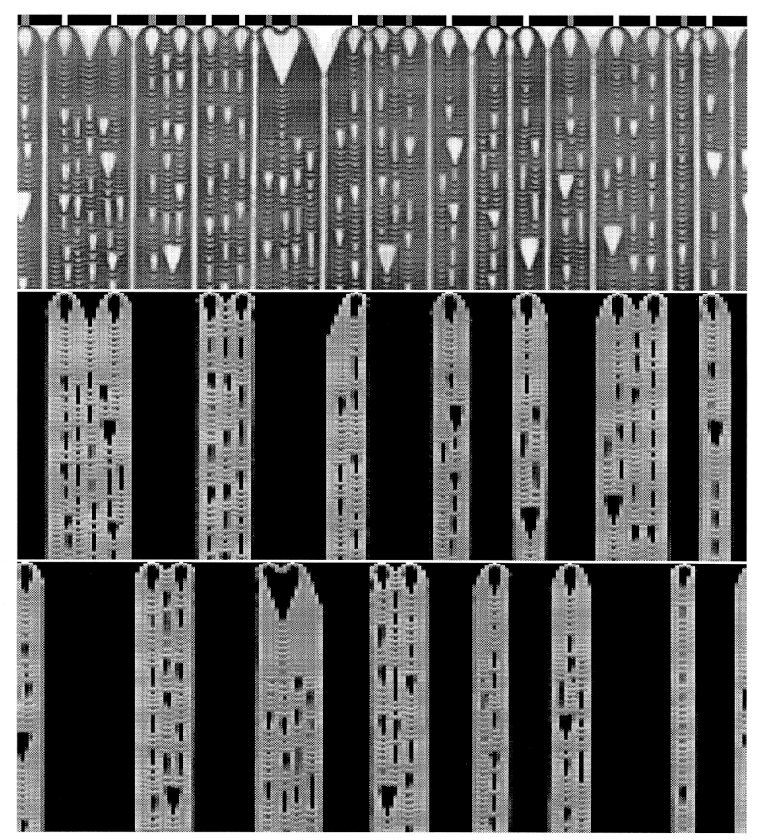

Figure 4.4. Spatiotemporal pattern of reactant concentration $\alpha$ (top), and autocatalyst $\beta$ (middle) and $\gamma$ (bottom) of Eq. (4.17) for $\delta=1, \phi=2.8$ and $\mu=$ 33.15. A concentration of $1(0)$ is represented in white (black). The reactant $A$ is initially distributed homogeneously, $\alpha=1$, over the entire domain. Species B and $\mathrm{C}$ are randomly seeded, indicated by the white $(\beta=1)$ and gray $(\gamma=1)$ rectangles at the top of the figure, with the following constraints: the seeds are each 20 grid points, they are separated by a minimum of 60 grid points, and there is a total of 200 occupied grid points per species. $\delta t=0.0003$ and $\mathrm{L}=600$ (total 2400 grid points). Each panel consists of 5000 layers, with each layer plotted every $200 \delta t$. Time increases from top to bottom in each panel. 


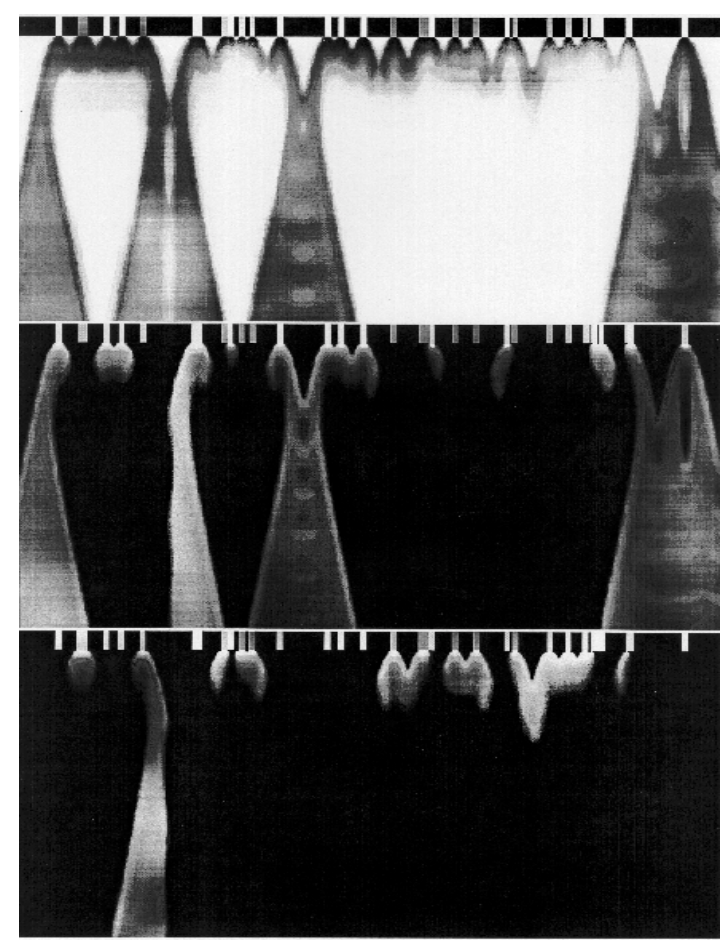

Figure 4.5. Transient spatiotemporal dynamics (300 layers) of reactant $\alpha$ (top), and autocatalysts $\beta$ (middle) and $\gamma$ (bottom) for a random distribution of initial seeds with $b=1$ and $c=1$, respectively, and a seed length of 10 grid points. The other parameters are the same as in Fig. 4.4. (R. Wackerbauer, H. Sun, and K. Showalter, unpublished.) 
Fig. 4.6 shows representative concentration profiles of reactant $A$ and autocatalysts $B$ and $C$ from Fig. 4.4. We see the transitions between domains as well as excursions away from the unstable focus $S_{-}{ }^{\text {B,C }}$ within the domains. The domain interfaces are characterized by a high concentration of $\mathrm{A}$ and low concentration of $B$ and $C$ as the wave fronts of each species intersect. While the exact profile at the interface depends on the system parameters and the behavior in adjacent domains, small fluctuations are typically observed around a state determined by continuity constraints of the neighboring steady states $S_{-}{ }^{\text {B,C }}$ (Fig. 4.7). Within a domain, the dynamical behavior can range from regular to chaotic, depending on the domain size and configuration of the initial local perturbations. For example, limit cycle behavior is exhibited for a periodic distribution of the initial perturbations and a relatively small domain size (65 grid points and $\mu=33.15$ ). In contrast, the behavior in Fig. 4.4, arising from random initial perturbations, gives rise to the chaotic trajectory shown in Fig. 4.7, which displays frequent excursions from the unstable focus $S_{-}{ }^{\text {B,C }}$ to approach the $S^{\mathrm{A}}$ state. This spatiotemporal chaos is much like that observed in the two-variable, single-autocatalyst system. ${ }^{17,20}$

Even though the domain boundaries appear to be completely localized on the time scale of the local dynamics within a domain, Fig. 4.4, the long-time behavior reveals irregular motions of these interfaces, as shown in Fig. 4.8. These motions, which occur only for chaotic populations, are most pronounced when the system is close to the wave propagation threshold where large-scale pulse structures are exhibited, such as in Figs. 4.4 and 4.8. The interfaces are characterized by a state that moves closer to the $S^{\mathrm{A}}$ state as the parameter $\mu$ is decreased. Concentration fluctuations at the interface are larger and injections of the trajectory to the neighborhood of the unstable focus are consequently more likely at the interface for smaller $\mu$. In contrast, steady-state behavior above the Hopf bifurcation point, $\mu>\mu_{\mathrm{H}}$, is accompanied by interfaces that are completely localized (spatially stationary) with constant concentrations $(\alpha, \beta, \gamma)$ for any random initial seeding. We also note that interface boundaries are always localized for symmetrical initial perturbations, even for chaotic populations, when the neighboring populations are dynamically in phase. Randomly seeded autocatalyst populations, on the 


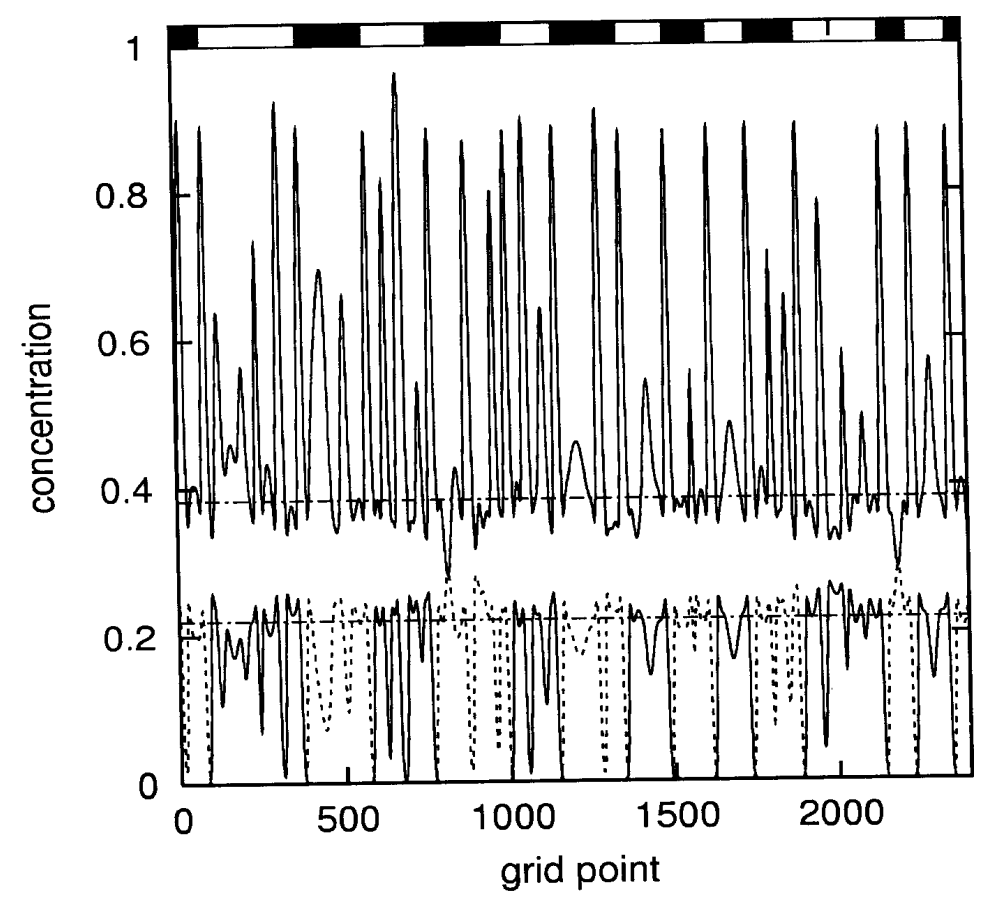

Figure 4.6. Representative concentration profile from Fig. 4.4 for reactant A (upper full line) and autocatalysts B (lower full line) and C (dotted line) taken at the final time step of Fig. 4.4. Horizontal lines show the concentrations $\alpha$ (upper), $\beta, \gamma$ (lower) at the unstable focus. Domains with $B$ $(C)$ activities are indicated by white (black) bars at top. (R. Wackerbauer, $\mathrm{H}$. Sun, and K. Showalter, Phys. Rev. Lett, 84, 5018 (2000).) 


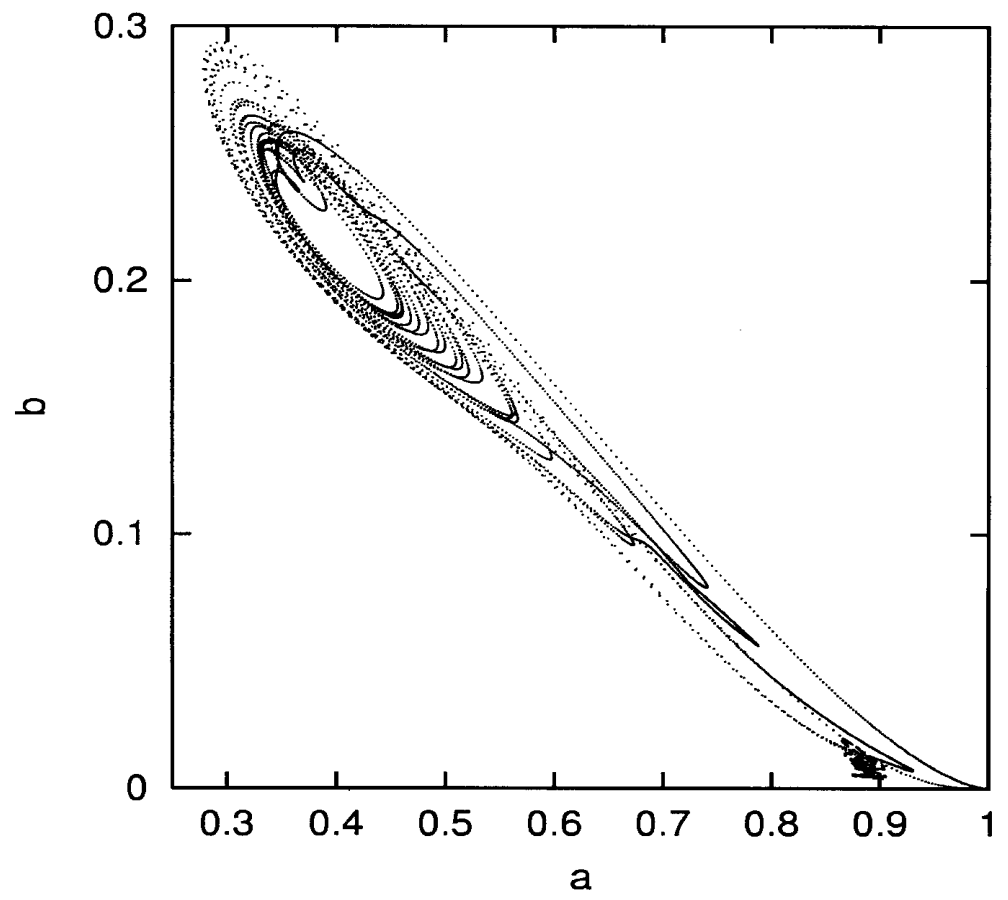

Figure 4.7. Characteristic phase portraits of the dynamics at a domain interface (full line localized near $\alpha=0.9$ ) and within a chaotic domain (dotted line) of Fig. 4.4. (R. Wackerbauer, H. Sun, and K. Showalter, Phys. Rev. Lett, 84, 5018 (2000).) 


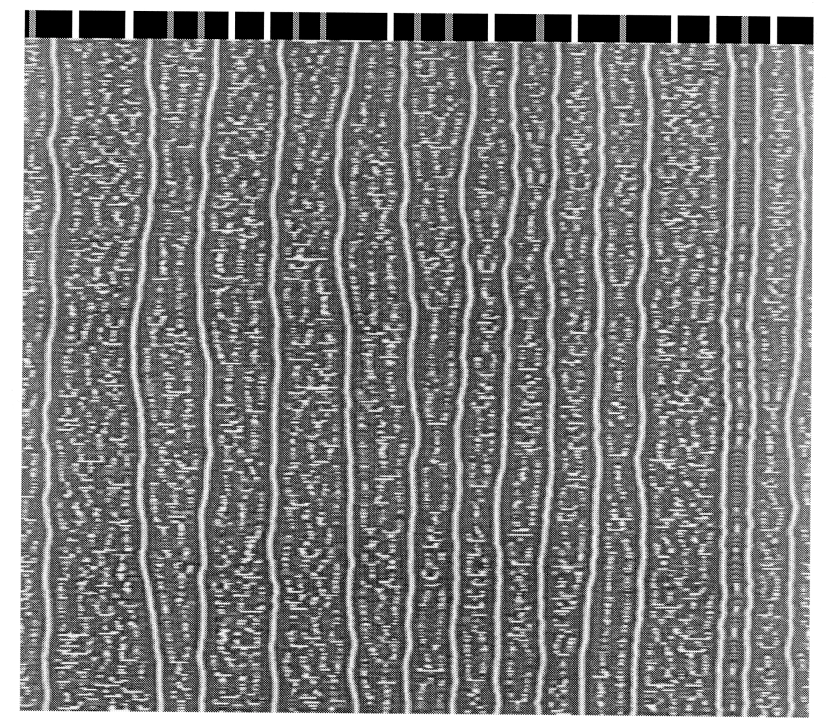

Figure 4.8. Long-term spatiotemporal dynamics of reactant concentration $\alpha$ for the dynamical system in Fig. 4.4. The panel consists of 2500 layers, with each layer plotted every 200008t. Other parameters are the same as in Fig. 4.4. (R. Wackerbauer, H. Sun, and K. Showalter, Phys. Rev. Lett, 84, 5018 (2000).) 
other hand, yield domains of neighboring populations with out-of-phase oscillations that generate spatially asymmetric perturbations to the interface.

The qualitative features of the self-segregation and domain interfaces are not dependent on the boundary conditions or the numerical method, although quantitative differences appear for no-flux and periodic boundary conditions. Furthermore, the qualitative features of the behavior are preserved when inputs of each autocatalyst are provided from outside reservoirs $\left(\beta_{0} \neq 0, \gamma_{0} \neq 0\right)$. In this case, however, a very low concentration of the "minor" species is always present in the domains. Additional considerations arise for predicting the appearance of segregation interfaces if we abandon the requirement of non-overlapping perturbations initiating autocatalysis. In this case, the autocatalytic species with the larger concentration survives at sites where overlapping perturbations have different concentrations. For equal initial concentrations, however, the reaction-diffusion waves of both species die out (even when $\beta+\gamma<1$ ). These results point to the existence of a planar separatrix in the phase space, defined by $\beta=\gamma$, which partitions the orthogonal manifolds of the competing autocatalysis subsystems. Trajectories starting on this separatrix move to the stable steady state $S^{\mathrm{A}}$. When the autocatalytic species have different diffusivities $\left(D_{B} \neq D_{\mathrm{C}}, D i \leq D_{\mathrm{A}}\right)$, the interfaces become less irregular and domains with the faster diffusing autocatalyst shrink, with the interfacial motion dependent on the difference in the diffusivities. An increase of the parameter $\mu$ for the inferior species, however, can compensate for the disadvantage in the diffusivity. Finally, we note that a significant qualitative change in behavior occurs when the reaction kinetics in Eq. (4.17) is modified from cubic to quadratic autocatalysis. The self-segregation is replaced by a coexistence of the two species (when they are governed by the same kinetics and diffusivities) throughout the medium. 


\subsubsection{Self-segregation in Two-dimensional Media}

Competitive autocatalysis can readily be generalized to two-dimensional media with two, three, or more species. As shown in Fig. 4.9, self-segregating populations are also found in two-dimensional media for the case of three competing autocatalytic species. The appearance of chaotic behavior and more ordered behavior in smaller domains, as well as the local concentration profiles at interfacial boundaries, are similar to those observed in the one-dimensional case, Fig. 4.6. A new feature appearing in two-dimensional media is the drift of domain boundaries such that convex curvature is reduced. For a closed interface this yields the collapse of a convex domain following a curve-shortening mechanism. ${ }^{15,16}$ Approximately circular domains obey a parabolic-like decrease in radius with time for chaotic as well as steady state $\left(\mu>\mu_{\mathrm{H}}\right)$ behavior. The interface drift occurs on a much slower time scale than that of the chaotic behavior within a domain.

Interface junctions, which occur with three or more competing species, as well as isolated interface loops and domains connected to the medium boundaries, can be seen in the segregation patterns shown in Fig. 4.9. The evolution shown in successive panels reveals curvature-induced shrinking and the ultimate extinction of domains, giving rise to the local breakup and reorganization of the interface junctions. Domains of the same species merge (sometimes only after a long and complex transient period) and small domains diminish in number during such reorganizations. The counterpart to the irregular interface motions for chaotic populations in one-dimensional media is not readily apparent in two-dimensional media, although slight motions in nearly linear interfaces are observed. For interfaces with significant curvature, however, interfacial drift dominates the behavior and the irregular interface motions are suppressed. Although the evolution of the interface reorganization varies with the parameter $\mu$, the qualitative features of the asymptotic states are very similar. Finally, we note that similar interface evolution has been found for competitive steady state populations. ${ }^{15}$ 

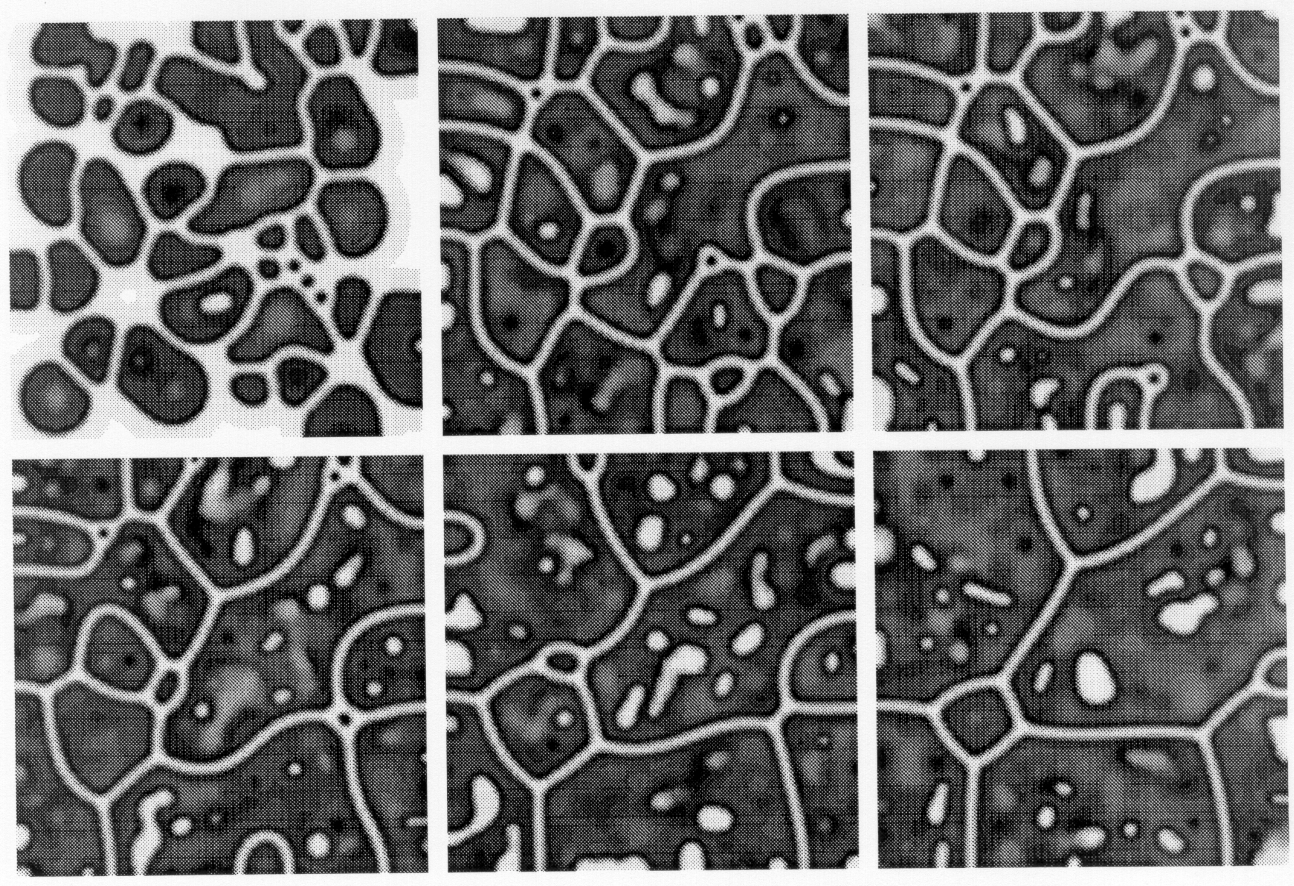

Figure 4.9. Spatiotemporal evolution of concentration $\alpha$ when three autocatalytic species compete for the reactant. From upper left: $t=41,176,239,364$, 655, and 1154. The white patches within the domains correspond to local reaction dynamics close to the extinct state. Initial conditions: 20 seeds of each species $(B, C, D)$ with a cell size of $20 \times 20$ grid points were randomly distributed on the array of $L \times L=$ $200 \times 200(800 \times 800$ grid points $)$ with a uniform reactant concentration $\alpha=1 . \delta t=$ 0.0052. Other parameters are the same as in Fig. 4.4. (R. Wackerbauer, H. Sun, and K. Showalter, Phys. Rev. Lett, 84, 5018 (2000).) 


\subsection{Conclusions}

In summary, the $\mathrm{n}$ species of reaction-diffusion systems such as Eq. (4.17) compete for a common sustaining resource and segregate into domains of uncorrelated, chaotic populations over a range of parameter values. Irregular motions of the interfaces occur due to the influence of out of phase oscillatory behavior of neighboring populations, while completely localized interfaces are observed for adjacent steady-state populations or in-phase oscillatory populations arising from symmetrical initial perturbations. In twodimensional media, curvature-induced interface drift gives rise to shrinking and, ultimately, extinction of domains as well as domain reorganizations arising from the breakup of interface junctions.

The self-segregation behavior found in this study is relevant to systems composed of species with similar characteristics, and we have focused on the case of identical diffusivities and growth rates of autocatalysis. Examples of such systems include competing populations in biological ecologies as well as isomers or isotopically labeled species in competing chemical reactions. As the diffusivities and rate constants are allowed to differ, the self-segregation behavior is retained only transiently, with the lifetime of the transient dependent on the similarity of the competing species.

Ever more complicated self-segregation behavior, with topological features determined by domain interface motions and the stability of interface junctions, should be found in the generalization of Eq. (4.17) to $n>3$ chaotic populations. 


\subsection{Appendix: The C Program for the Simulations}

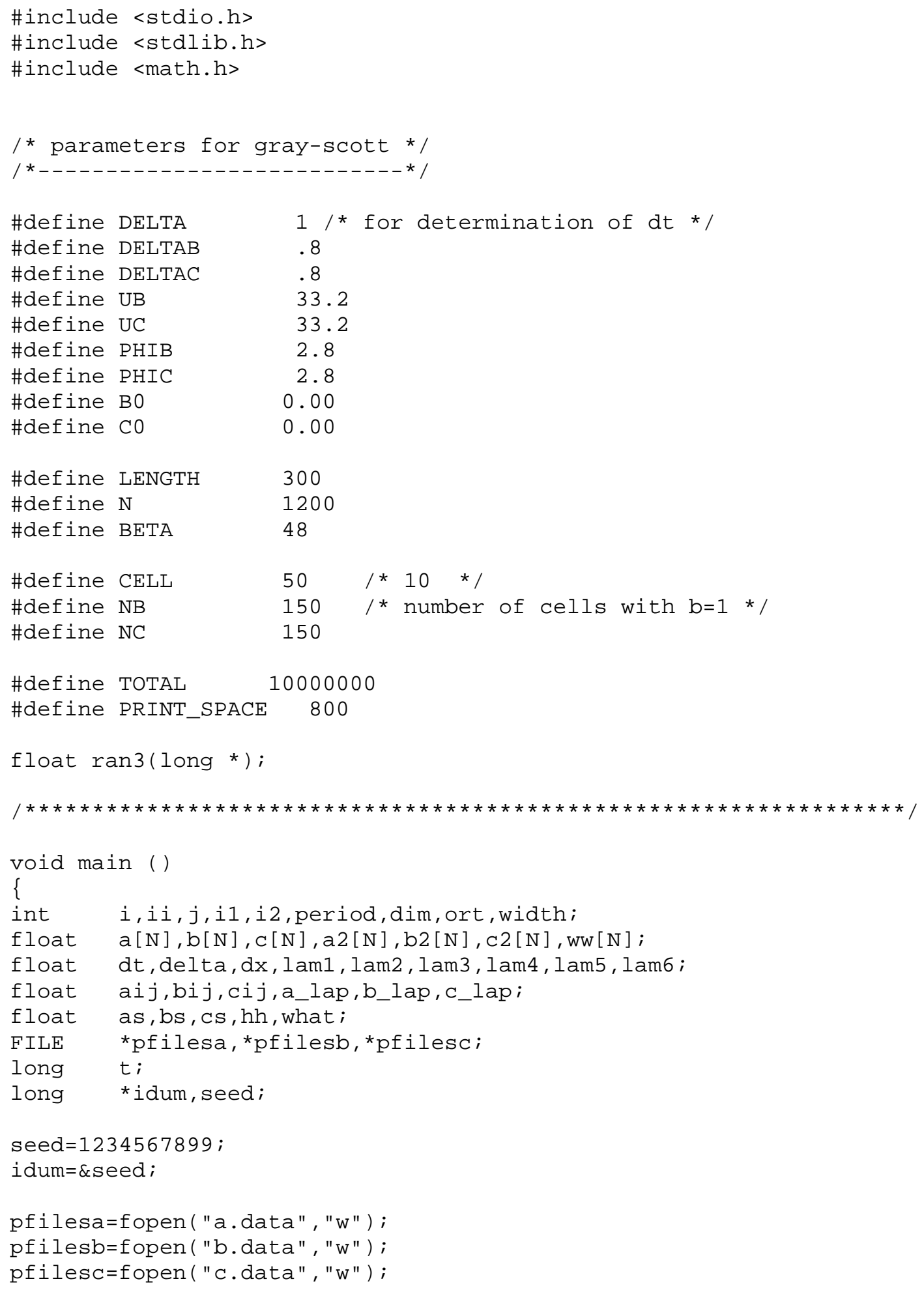




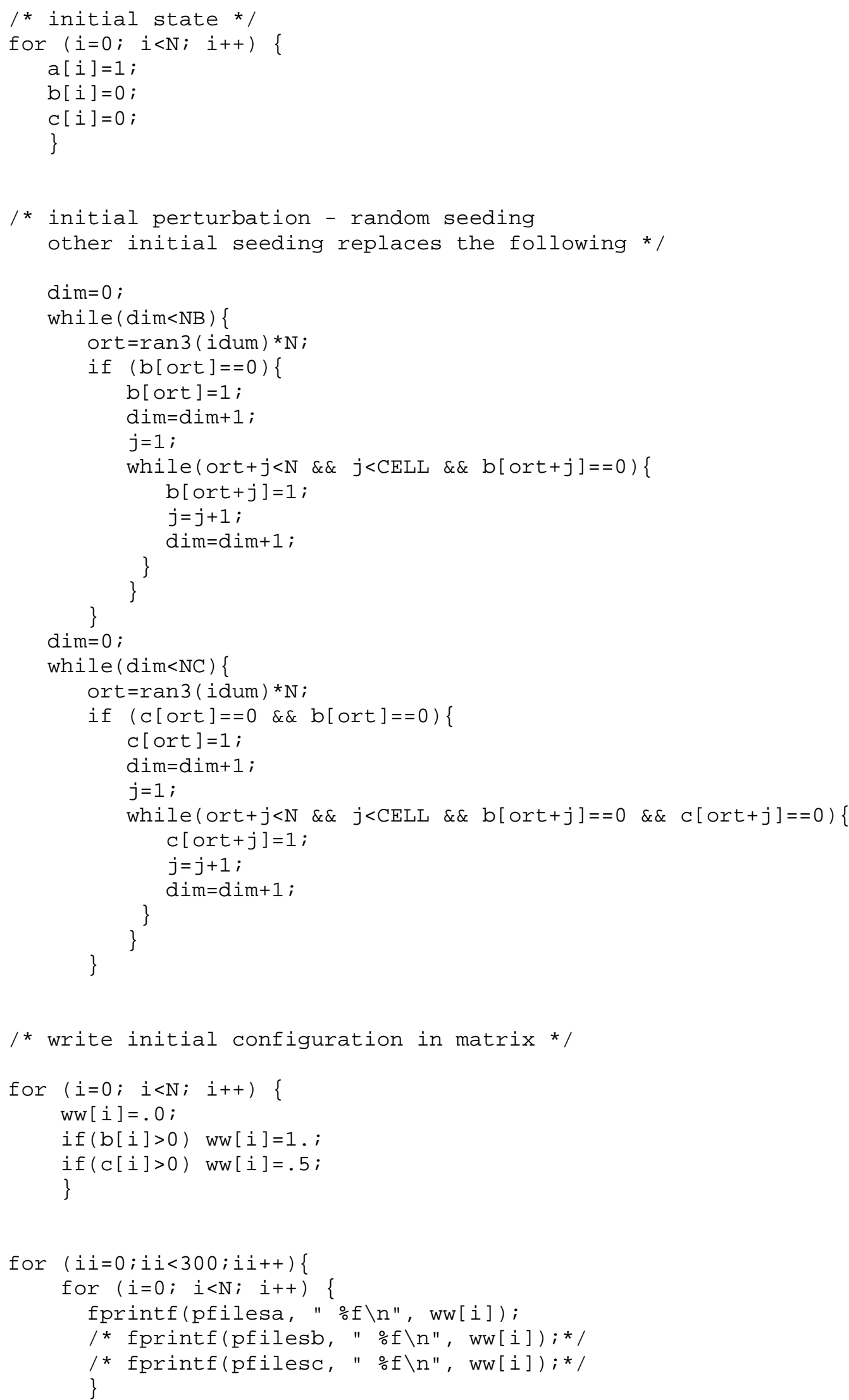




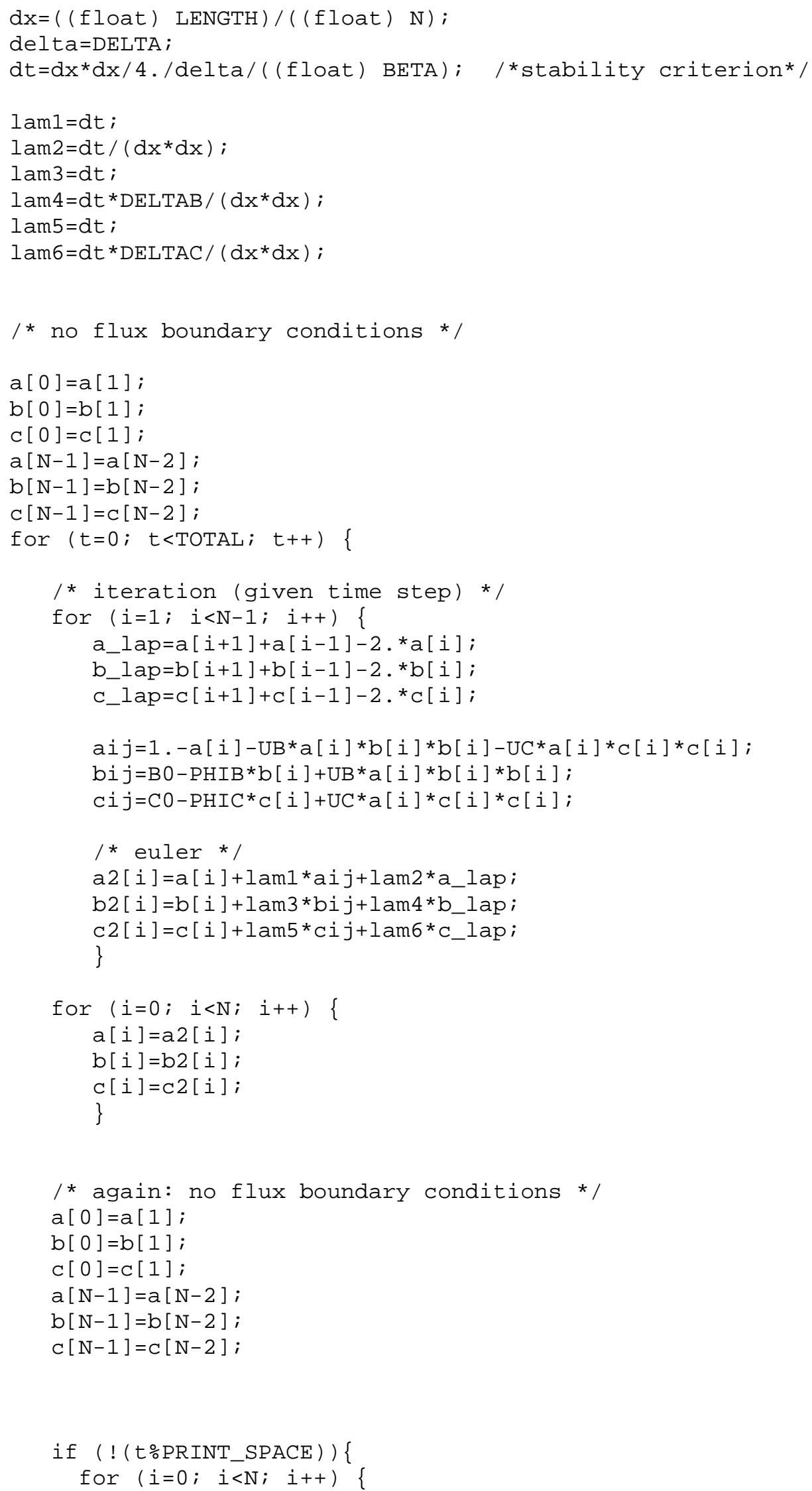




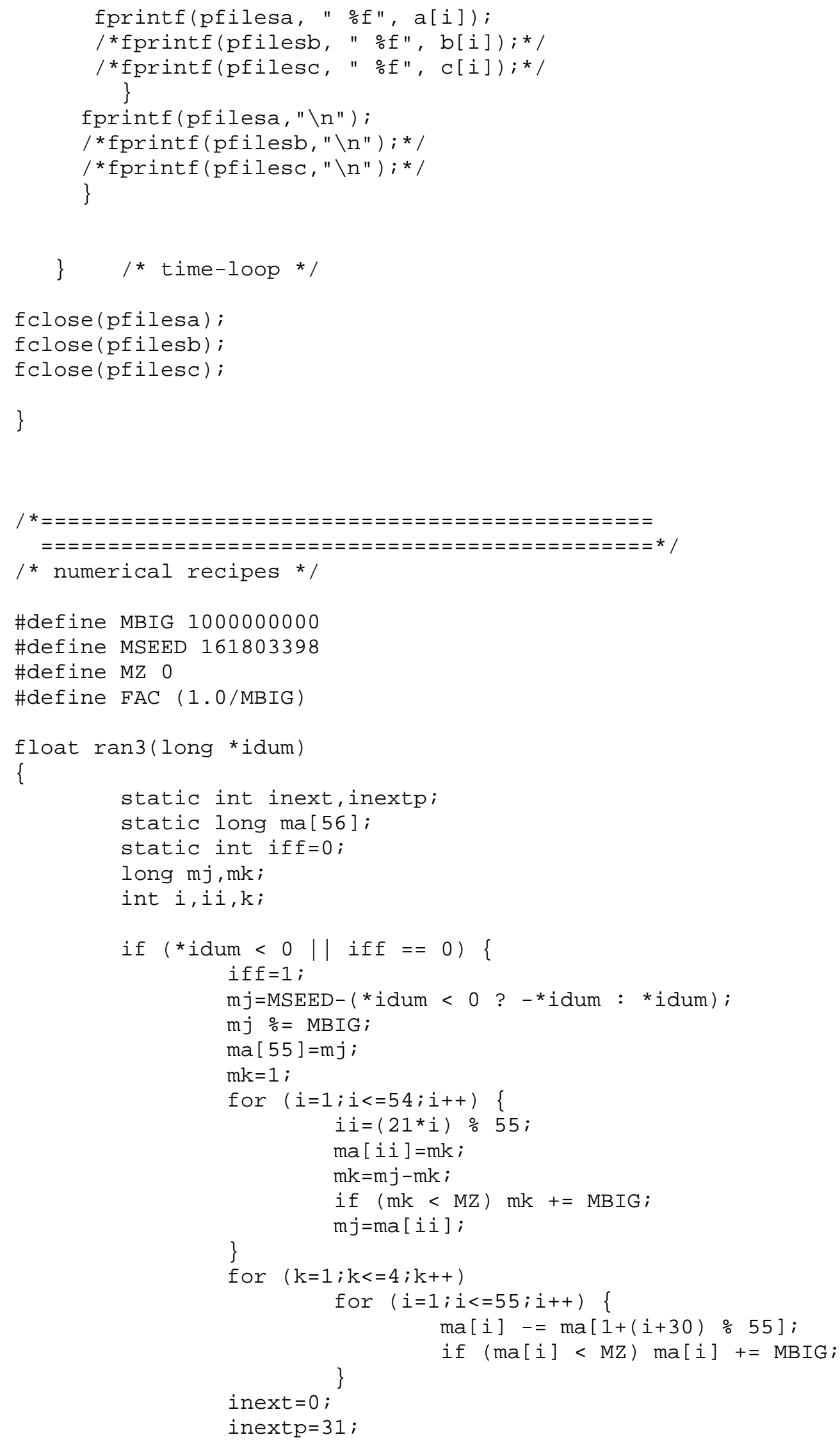




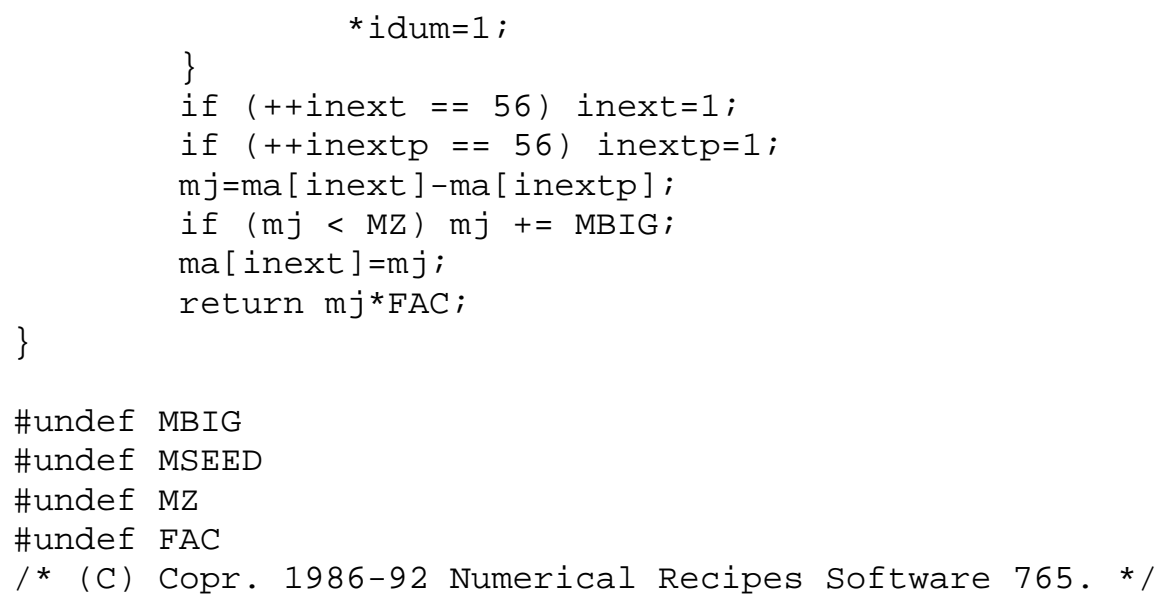




\subsection{References}

1. J. D. Murray, Mathematical Biology, (Spring-Verlag, Berlin, Heidelberg, 1989).

2. M. Bulmer, Theoretical Evolutionary Ecology, (Sinauer Associates, Sunderland, Massachusetts, 1994).

3. H. Haken, Advanced Synergetics (Springer, Berlin, Heidelberg, 1983).

4. E. Scholl, Nonequilibrium Phase Transitions in Semiconductor: Self-Organization induced by Generation and Recombination Processes (Spring-Verlag, Berlin, Heidelberg, 1987).

5. G. Nicolis and I. Prigogine, Self-Organization in Non-Equilibrium Systems (Wiley, New York, 1997).

6. J. Hofbauer and K. Sigmund, Evolutionary Games and Population Dynamics, (Cambridge University Press, Oxford, 1998).

7. F. T. Arecchi, S. Boccaletti, G. P. Puccioni, P. L. Ramazza, and S. Residori, Chaos 4, 491 (1994).

8. S. Havlin, M. Araujo, H. Larralde, H. E. Stanley, and P. Trunfio, Physica (Amsterdam) 191A, 143 (1992).

9. A. Yen and R. Kopelman, Phys. Rev. E 56, 3694 (1997).

10. R. Reigada, F. Sagues, I. M. Sokolov, J. M. Sancho, and A. Blumen, J. Chem. Phys. 107, 843 (1997).

11. J. H. Merkin, A. J. Poole, S. K. Scott, J. Masere, and K. Showalter, J. Chem. Soc. Faraday Trans. 94, 53 (1994).

12. G. Hardin, Science 131, 1292 (1961).

13. J. Huisman and F. J. Weissing, Nature (London) 402, 407 (1999).

14. R. Wackerbauer, H. Sun, and K. Showalter, Phys. Rev. Lett. 84, 5018 (2000).

15. S. -I. Ei, R. Ikota, and M. Mimura (to be published), and references therein. 
16. M. Gage and R. S. Hamilton, J. Differ. Geom. 23, 69 (1986).

17. P. Gray and S. K. Scott, Chem. Eng. Sci. 39, 1087 (1994).

18. J. E. Pearson, Science 261, 189 (1993).

19. K. J. Lee, W. D. McCormick, J. E. Pearson, and H. L. Swinney, Nature (London) 369, 215 (1994).

20. J. H. Merkin, V. Petrov, S. K. Scott, and K. Showalter, Phys. Rev. Lett. 76, 546 (1996).

21. J. H. Merkin, V. Petrov, S. K. Scott, and K. Showalter, J. Chem. Soc. Faraday Trans. 92, 2911 (1996).

22. J. H. Merkin and M. A. Sadiq, IMA J. Appl. Math. 57, 273 (1996). 


\section{Chapter 5}

\section{Transition from Segregation to Integration of Chaotic Populations}

\subsection{Introduction}

In physical and chemical areas, ${ }^{1-7}$ nonlinear generation-recombination processes are very common and often produce cooperative effects such as chaotic oscillations among all participating particles, carriers or various field quantities. Examples for such behavior

have been found in semiconductor, ${ }^{4}$ plasma, ${ }^{5}$ optics, ${ }^{6}$ and other systems. ${ }^{2,7-9}$ For example, chaotic oscillations in a two-carrier (electron-hole) plasma have been reported in Ge at $77 \mathrm{~K}$ in parallel electric and magnetic fields, ${ }^{10}$ where the oscillation of one carrier affects that of the other carrier and they together contribute to the chaotic character of the measured signals in discharge current and spatially dependent potential.

In general, the chaotic oscillations of various quantities in a given system will be out of phase. As we have discussed in the previous chapter, the chaotic oscillations of a given component may be accompanied by the extinction of another component, i.e., the system may exhibit the phenomenon of segregation as a result of a competition between different species. Moreover, it has been observed in a variety of social and physical systems that cooperation and competition can coexist, and, hence, in such systems a transition from segregation to integration might be expected if the parameters are varied in a suitable 
way. Here, integration means that two or more species can coexist everywhere, although one species may be dominant at a particular time and place. This is in contract to the phenomenon of segregation as discussed in Chapter 4, where species cannot coexist within any local domain and thus segregate with domain walls separating them. ${ }^{1}$ As already mentioned in Chapter 4, homogenous coexistence can be found in a system with quadratic competitive reactions, but the integration of species with chaotic dynamics or a transition from segregation to integration is not observed. Here we present a model that exhibits both phenomena. Our study is also relevant to biology and ecology, because different species may rely on each other in other ways than the prey-predator relation, for example, by mating to proliferate subsequent generations. ${ }^{11-13}$

In Chapter 4, we discussed the phenomenon of segregation based on competition. The organization of this chapter is similar: In Section 5.2, we introduce the model and we perform a linear stability analysis of the homogeneous solutions in Section 5.3. In Section 5.4, numerical results are presented and discussed and we summarize and draw our conclusions in Section 5.5.

\subsection{The Model}

In addition to the competing processes of self-replication as expressed by $A+2 B \rightarrow 3 B$ and $A+2 C \rightarrow 3 C$, we introduce the cooperative processes, $A+B+C \rightarrow 2 B+C$ and $A+B+C \rightarrow B+2 C$. Here, $B$ and $C$ together consume $A$ and produce more $B$ or $C$, and thus each of the new reactions implies cooperation between $B$ and $C$. However, the cooperative relations, $A+B+C \rightarrow 2 B+C$ and $A+B+C \rightarrow B+2 C$, also have a competitive aspect: While the former reaction favors the production of $B$, the latter favors $C$. This is in fact a selective process, and it contributes to the imbalance between the equilibrium concentrations of species if the two steps have different reaction rates. 
Following the discussion, the central reaction steps can be summarized as follows:

$$
\begin{array}{ll}
A+2 B \rightarrow 3 B & \text { rate }=k_{B} a b^{2}, \\
A+2 C \rightarrow 3 C & \text { rate }=k_{C} a c^{2}, \\
A+B+C \rightarrow 2 B+C & \text { rate }=k_{B C} a b c \\
A+B+C \rightarrow B+2 C & \text { rate }=k_{C B} a b c
\end{array}
$$

These four reactions form the competition-cooperation processes and, because they are cubic in character, the reactions provide sufficient nonlinearity for the system to exhibit nontrivial complex behavior. These reactions keep their symmetry from the point of view of qualitative description because one reaction will not dominate over the others without incorporating other factors that affect the symmetry. Quantitatively, we expect that any imbalance among the concentrations can be attributed to the relative variation of the rates of these reactions. For example, if the process of $A+B+C \rightarrow B+2 C$ has a larger rate than that of $A+B+C \rightarrow 2 B+C$ at a particular place and time, $C$ may be produced in a larger quantity in comparison to the species $B$.

Besides the four central reaction steps, we note, as addressed in the previous chapter, that there exist independent exchanges of reactants $A, B$, and $C$ between the reaction zone and the reservoirs. The exchange or flow rate is denoted by a constant $k_{f}$, which is controllable in experiments. $B$ and $C$ decay as $B \rightarrow P$ and $C \rightarrow Q$, where $P$ and $Q$ are inertial products and will eventually be removed from the reaction zone. For simplicity, we assume that the decay processes have identical rate constants $k_{d}$. These auxiliary steps provide linear terms in the corresponding reaction-diffusion equations and are also necessary in supporting the complex dynamics of the system.

Based on the above reaction steps, we can obtain a set of partial differential equations (PDEs). Introducing dimensionless space and time and rescaling the variables and parameters, we can express the PDEs as follows: 


$$
\begin{aligned}
& \frac{\partial \alpha}{\partial \tau}=\delta \nabla^{2} \alpha+1-\alpha-\alpha\left(\mu_{B} \beta^{2}+\mu_{C} \gamma^{2}\right)-\left(v_{B}+v_{C}\right) \alpha \beta \gamma \\
& \frac{\partial \beta}{\partial \tau}=\sigma \nabla^{2} \beta-\phi \beta+\mu_{B} \alpha \beta^{2}+v_{B} \alpha \beta \gamma \\
& \frac{\partial \gamma}{\partial \tau}=\nabla^{2} \gamma-\phi \gamma+\mu_{C} \alpha \gamma^{2}+v_{C} \alpha \beta \gamma
\end{aligned}
$$

where $\alpha=a / a_{0}, \beta=b / a_{0}, \gamma=c / a_{0}, \tau=t / t_{e x}$ with $t_{e x}=1 / k_{f}, x=X / L$ with $L=\sqrt{ }\left(D_{C} t_{e x}\right), \mu_{B}=$ $k_{B} a_{0}^{2} / k_{f}, \mu_{C}=k_{C} a_{0}^{2} / k_{f}, v_{B}=k_{B C} a_{0}^{2} / k_{f}, v_{C}=k_{C B} a_{0}^{2} / k_{f}, \phi=\left(k_{f}+k_{d}\right) / k_{f}, \delta=D_{\mathrm{A}} / D_{\mathrm{C}}$ and $\sigma=D_{\mathrm{B}} / D_{\mathrm{C}}$ (see Section 4.2 and 4.3). For the sake of simplicity, we assume equal diffusivities for $A$, $B$ and $C$ as well as identical rates for the autocatalytic and the decay processes. These simplifications can be justified in certain biological or chemical contexts but do not necessarily apply to all possible situations. Here, Eqs. (5.2) simplify to:

$$
\begin{aligned}
& \frac{\partial \alpha}{\partial \tau}=\nabla^{2} \alpha+1-\alpha-\mu \alpha\left(\beta^{2}+\gamma^{2}\right)-\left(v_{B}+v_{C}\right) \alpha \beta \gamma \\
& \frac{\partial \beta}{\partial \tau}=\nabla^{2} \beta-\phi \beta+\mu \alpha \beta^{2}+v_{B} \alpha \beta \gamma \\
& \frac{\partial \gamma}{\partial \tau}=\nabla^{2} \gamma-\phi \gamma+\mu \alpha \gamma^{2}+v_{C} \alpha \beta \gamma
\end{aligned}
$$

Within the focus of the study, the difference between the parameter $v_{\mathrm{B}}$ and $v_{\mathrm{C}}$ implies an imbalance of the selective processes, which directly results in a difference of the equilibrium concentrations of $B$ and $C$. We point out that the extinction of one species, either $B$ or $C$, reduces the system to the Gray-Scott model, ${ }^{15-17}$ in which chaotic spatiotemporal behavior can be found over a certain range of parameters. This is important because the underlying chaos of the Gray-Scott model will result in a system with chaotic populations. ${ }^{18}$ In order to extend the analysis of the simplified model (5.3), we will briefly discuss the more general case involving different diffusivities as well as different $\mu_{B}$ and $\mu_{C}$ the end of this chapter. 


\subsection{Linear Stability Analysis}

We first determine the homogenous steady states of Eq. (5.3) and their stabilities for various values of parameters. Three sets of steady states coincide with those discussed in Section 4.3: $S^{A}=(1,0,0), S_{ \pm}^{B}=\left(a_{ \pm}, h_{ \pm}, 0\right)$ and $S_{ \pm}{ }^{C}=\left(a_{ \pm}, 0, h_{ \pm}\right)$, where $a_{ \pm}=$ $\left(1 \pm \sqrt{1-4 \phi^{2} / \mu}\right) / 2$ and $h_{ \pm}=\phi /\left(\mu a_{ \pm}\right) . S^{A}$ represents the "extinguished" steady state, which is locally stable with respect to homogeneous perturbations in the entire parameter space. Although the existence of ${S_{ \pm}}^{B}$ and $S_{ \pm}{ }^{C}$ is independent of $v_{B}$ and $v_{C}$ (or $v$ when $v \equiv v_{B}$ $=v_{C}$ ), their stability does depend on these two parameters. The stability of each steady state is determined by the solution of the characteristic equation for the Jacobian matrix for the homogeneous system of Eq. (5.3),

$$
J_{s s}=\left(\begin{array}{ccc}
-\left[1+\mu\left(\beta^{2}+\gamma^{2}\right)+\left(v_{B}+v_{C}\right) \beta \gamma\right] & -\left[2 \mu \alpha \beta+\left(v_{B}+v_{C}\right) \alpha \gamma\right] & \left.-\left[2 \mu \alpha \gamma+\left(v_{B}+v_{C}\right) \alpha \beta\right]\right) \\
\mu \beta^{2}+v_{B} \beta \gamma & -\phi+2 \mu \alpha \beta+v_{B} \alpha \gamma & v_{B} \alpha \beta \\
\mu \gamma^{2}+v_{C} \beta \gamma & v_{C} \alpha \gamma & -\phi+2 \mu \alpha \gamma+v_{C} \alpha \beta
\end{array}\right.
$$

When $v_{B}=v_{C}=0, S_{ \pm}{ }^{B, C}$ exist for $\mu$ above the saddle-node bifurcation $\mu_{\mathrm{sn}}=4 \phi^{2} . S_{+}{ }^{B, C}$ are saddles and $S_{-}^{B, C}$ are nodes or foci when viewed in the plane perpendicular to the attracting eigen-direction, which is associated with the negative eigenvalue $\lambda_{\perp}=-\phi . S_{-}^{B, C}$ experiences a Hopf bifurcation at $\mu_{H}=\phi^{4} /(\phi-1)$ when $\phi>2$. Depending on $\phi$, the Hopf bifurcation can be either supercritical or subcritical (see Section 4.3). As we have discussed in Chapter 4, the coexistence of $S_{-}{ }^{B}$ and $S_{-}{ }^{C}$ causes the segregation of species $B$ and $C$, because the solutions of $S_{-}{ }^{B}$ and $S_{-}{ }^{C}$ imply the mutual exclusion of species $B$ and $C$. Besides $S^{A}, S_{ \pm}{ }^{B}$ and ${S_{ \pm}}_{ \pm}$, other steady states $S_{ \pm}{ }^{I}$ exist which are determined by 


$$
\begin{aligned}
& \alpha^{ \pm}=\frac{1 \pm \sqrt{1-4\left[\mu\left(k_{\beta}^{2}+k_{\gamma}^{2}\right)+\left(v_{B}+v_{C}\right) k_{\beta} k_{\gamma}\right]}}{2}, \\
& \beta^{ \pm}=k_{\beta} / \alpha^{ \pm} \\
& \gamma^{ \pm}=k_{\gamma} / \alpha^{ \pm}
\end{aligned}
$$

where $k_{\beta}$ and $k_{\gamma}$ are given by:

$$
\begin{aligned}
& k_{\beta}=\frac{\phi\left(\mu-v_{B}\right)}{\mu^{2}-v_{B} v_{C}}, \\
& k_{\gamma}=\frac{\phi\left(\mu-v_{C}\right)}{\mu^{2}-v_{B} v_{C}} .
\end{aligned}
$$

A simplified analysis for the existence of $S_{ \pm}^{I}$ can be performed: For $v=v_{B}=v_{C}$ we have

$$
\alpha^{ \pm}=\frac{1 \pm \sqrt{1-8 \phi^{2} /(\mu+v)}}{2}
$$

so the existence of $S_{ \pm}^{I}$ (correspondingly, $\alpha^{ \pm}, \beta^{ \pm}$and $\gamma^{ \pm}$) is determined by the condition (cf. Fig. 5.1)

$$
0<8 \frac{\phi^{2}}{\mu+v}<1
$$

For $\phi \sim 2.8$ and $\mu \sim 34$, we have $v \geq 29$. In this study, we mainly focus on the spatiotemporal evolution of the spatially extended system around $v=34$, where a critical transition from segregation to integration occurs. 


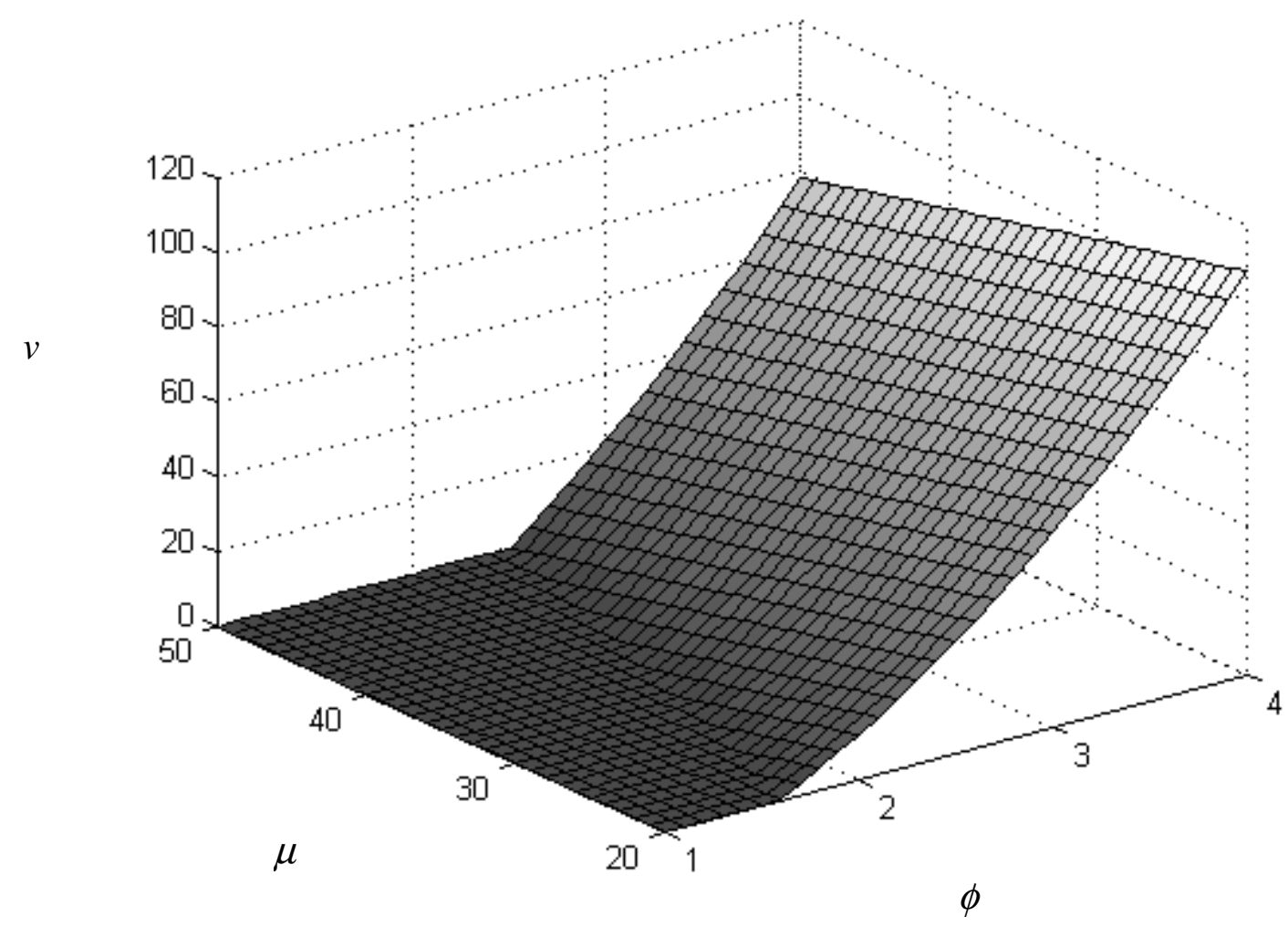

Figure 5.1. The critical surface determining the existence of $S_{ \pm}{ }^{I}$ in the case of $v=v_{\mathrm{B}}=v_{\mathrm{C}}: S_{ \pm}{ }^{I}$ only exist under the parametric conditions above the surface. 


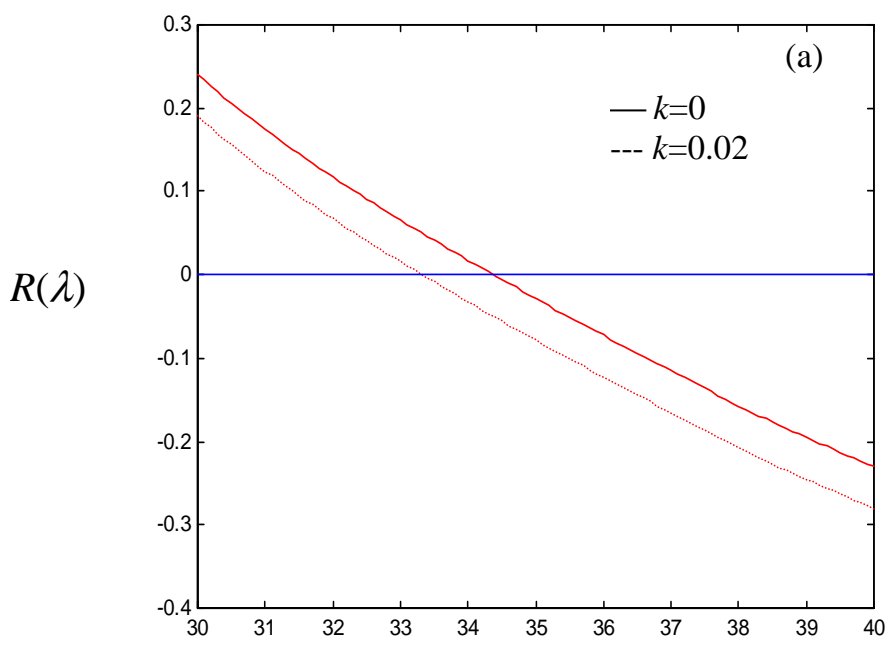

$v$

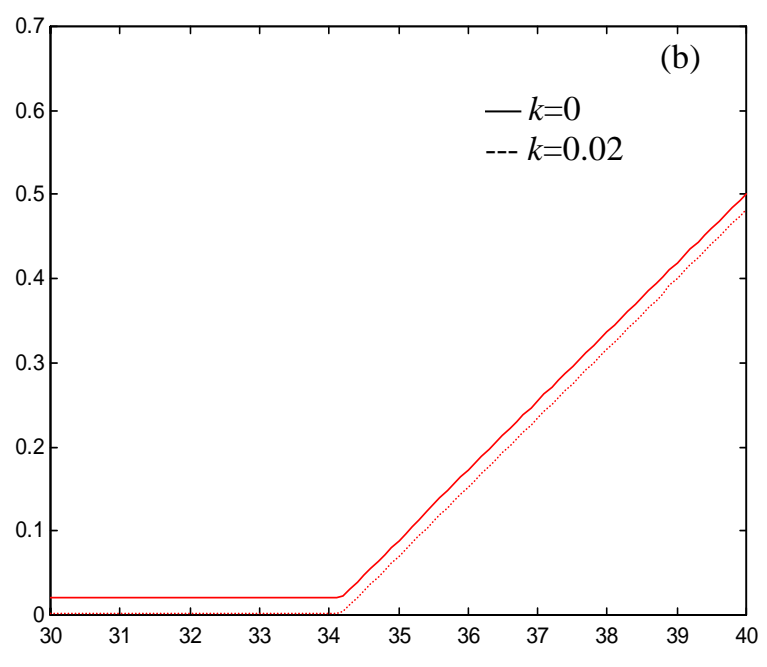

$v$

Figure 5.2. Real part $R(\lambda)$ of the largest eigenvalue for the homogeneous steady states (a) $S_{-}{ }^{I}$ and (b) $S_{-}{ }^{B, C}$ with $v=v_{B}=v_{C} . R(\lambda)$ represents the real part of the largest eigenvalue. $k$ is the wave number of a spatial mode. Here, $\mu=33.9$ and $\phi=2.8$, and the critical value $v_{c}$ appears around 34.1 for $k=0$. 


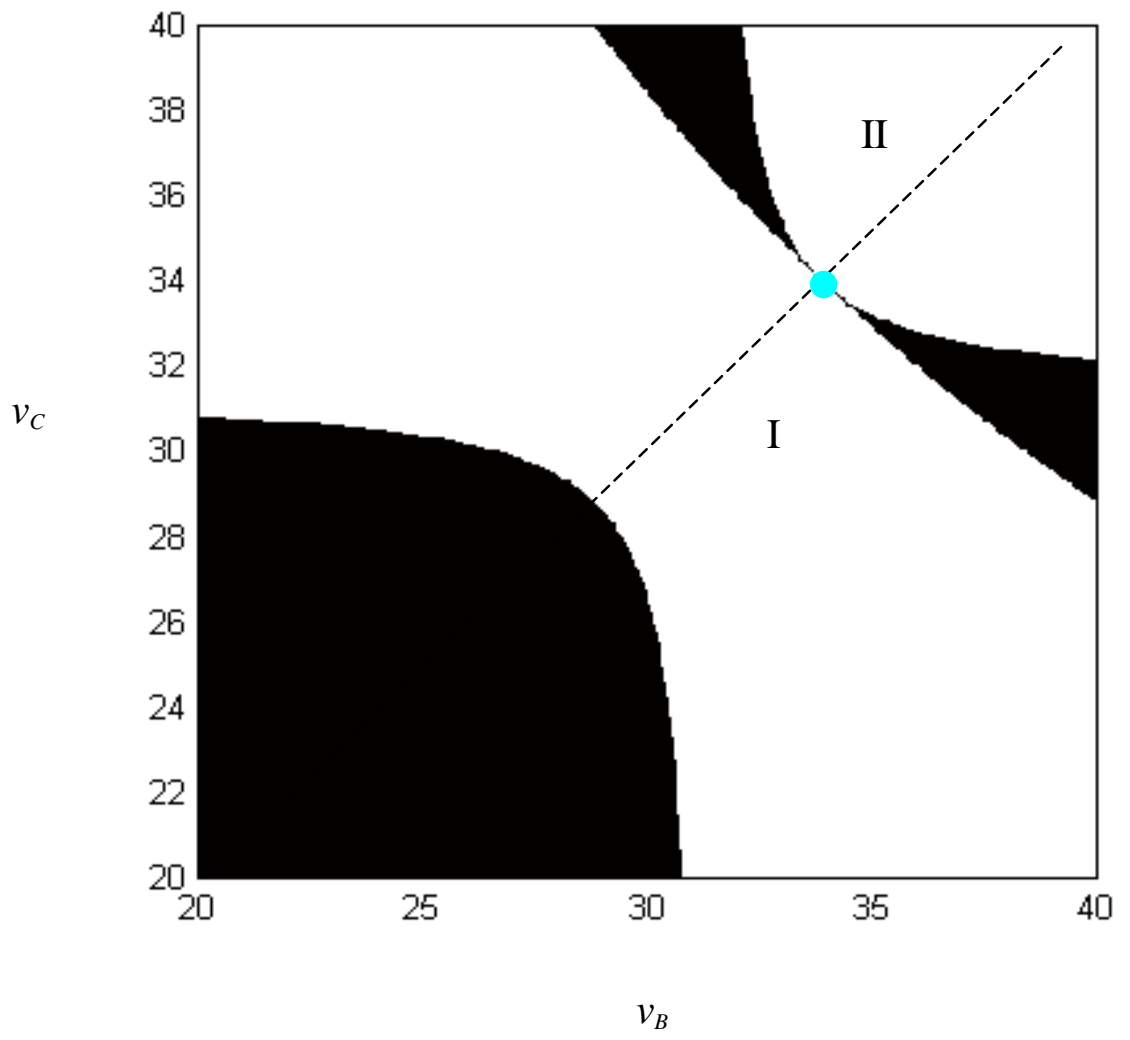

Figure 5.3. The existence and stability of $S_{-}{ }^{I}$ in the $2 \mathrm{D}$ parameter plane $v_{B}$ vs. $v_{C}$. No solutions exist in the black area. Regions where $S_{-}{ }^{I}$ is unstable and stable are marked by I and II, respectively. Inside I, chaotic segregation is dominant, while in region II, the system relaxes to the steady state corresponding to homogenous integration. Chaotic integration is found in the vicinity of the gray dot. 
The steady state $S_{+}{ }^{I}$ is a saddle within the parameter range of interest and is not relevant to asymptotic dynamics. As we see from the solution of $S_{-}{ }^{I}$, both $\beta^{-}$and $\bar{\gamma}$ do not vanish, which implies the coexistence of $B$ and $C$ in that steady state, i.e., it corresponds to homogeneous integration. Fig. 5.2(a) shows the real part of the largest eigenvalue of $S_{-}{ }^{I}$ for the case $v=v_{B}=v_{C}$, and we see that $S_{-}{ }^{I}$ becomes stable as the parameter $v$ crosses the critical value $v_{\mathrm{c}} \approx 34.1$. Fig. 5.2(b) shows the real part of the largest eigenvalue for the homogeneous steady states $S_{-}{ }^{B, C}$ : These states are unstable for all values of $v$, however the real part of the largest eigenvalue begins to increase as $v_{\mathrm{c}}$ is crossed.

In Fig. 5.3, a two-dimensional (2D) plot displays the parameter ranges for the existence and stability of $S_{-}{ }^{I}$. As we find in one-dimensional simulations of the PDE (as discussed in the next section), in region II, the system always relaxes to the homogeneous steady state $S_{-}{ }^{I}$, i.e., to stationary homogenous integration. For the case $v=v_{B}=v_{C}$, we find chaotic integration in the vicinity of the gray dot (corresponding to $v=v_{\mathrm{c}}$ ) shown in Fig. 5.3, i.e., the system relaxes to a spatiotemporally chaotic state and, specifically, every spatial element exhibits chaotic oscillations in both species $B$ and $C$. In general, however, different elements oscillate out of phase. As $v$ is further decreased below $v_{\mathrm{c}}$ in the symmetric case, the system relaxes to spatial segregation as described in Chapter 4. Hence, we find a transition from segregation to integration when $v$ passes the critical value $v_{\mathrm{c}}$.

In the asymmetric case $v_{\mathrm{B}} \neq v_{\mathrm{C}}$, we observe a direct transition from the segregated state to homogeneous stationary integration at the lower boundary of region II, i.e., no chaotic integration is found far enough away from the point marked by the gray dot in Fig. 5.3. Note that in the black areas shown in this figure, where $S_{-}{ }^{I}$ does not exist, the system always relaxes to a segregated state, except for a small range of initial conditions for which both species become extinct. 
The linear stability analysis we have performed so far for Eqs. (5.3) was limited to homogenous perturbations. It can be generalized to non-uniform perturbations with spatial period $\tilde{\lambda}_{k}=2 \pi / k$ : Setting $\alpha=\alpha_{s s}+\delta \alpha e^{i k x}, \quad \beta=\beta_{s s}+\delta \beta e^{i k x}, \quad \gamma=\gamma_{s s}+\delta \gamma e^{i k x}$, and linearizing around the homogenous steady state, we obtain the following Jacobian:

$$
J^{k}{ }_{s s}=\left(\begin{array}{ccc}
-k^{2}-\left[1+\mu\left(\beta^{2}+\gamma^{2}\right)+\left(v_{B}+v_{C}\right) \beta \gamma\right] & -\left[2 \mu \alpha \beta+\left(v_{B}+v_{C}\right) \alpha \gamma\right] & \left.-\left[2 \mu \alpha \gamma+\left(v_{B}+v_{C}\right) \alpha \beta\right]\right) \\
\mu \beta^{2}+v_{B} \beta \gamma & -k^{2}-\phi+2 \mu \alpha \beta+v_{B} \alpha \gamma & v_{B} \alpha \beta \\
\mu \gamma^{2}+v_{C} \beta \gamma & v_{C} \alpha \gamma & -k^{2}-\phi+2 \mu \alpha \gamma 4 v_{C} \alpha \beta
\end{array}\right.
$$

This implies the following relationship between the eigenvalues of Eqs. (5.10) and those of Eqs. (5.4):

$$
\lambda_{k}=\lambda_{k=0}-k^{2}
$$

Hence, the stability of homogeneous steady state $S_{-}{ }^{I}$ will in general depend on the wave number $k$ of a given spatial periodic infinitesimal perturbation. In the symmetric case for example, as the parameter $v$ is decreased beyond $v_{\mathrm{c}}, S_{-}{ }^{I}$ first becomes unstable with respect to homogeneous perturbations and only later undergoes an instability with respect to spatially periodic perturbations characterized by a certain wave number $k$.

\subsection{Numerical Simulation and Analysis}

\subsubsection{One-dimensional Simulation}


One-dimensional numerical simulations were performed in a system with size $L=200$, using 800 grid points (see Fig. 5.4). The integration step is $\delta t=0.01$ and the total evolution time $t=50000 \delta$. We choose $\phi=2.8$ and $\mu=33.9$ throughout this study, and no-flux boundary conditions were used. Initially, we set the concentration of the resource $\alpha=1$ everywhere and for each of the species $B$ and $C$ we seeded more than half of the sites, setting $\beta=1$ and $\gamma=1$ at the seeded site. Numerical simulations show that such choices of boundary and initial conditions are representative and alternative choices do not change the qualitative dynamic behavior except for a very narrow range of initial conditions, for which the system relaxes to the extinguished homogeneous stationary state.

Segregation occurs in the case of low values of $v$ and is characterized by the mutual exclusion of species in separated spatial domains [see Fig. 5.4 (c)]. As discussed in Chapter 4, the existence of the separated spatial domains reflects the coexistence of steady states $S_{-}{ }^{B}$ and $S_{-}{ }^{C}$ and their mutual exclusion of the two species $B$ and $C$. The domain walls representing high concentration of $\alpha(\sim 0.9)$ do not change in magnitude, however their position in space varies. This variation is induced by the instant phase asymmetry of the chaotic oscillations on each side of a domain wall. However, it is important to note that the domain walls do not disappear, provided that $v$ is sufficiently smaller than its critical value $v_{\mathrm{c}} \sim 34$. It is integration that removes the tendency of mutual exclusion and allows the coexistence of species anywhere in the system.

Close to but below $v_{\mathrm{c}}$, the domain walls eventually disappear, despite their initial formation. Following a transient period, the system relaxes to spatiotemporal chaotic dynamics with both species coexisting in the entire system [chaotic integration, cf. Figs. 5.4 (a) and (b)]. As $v$ exceeds the critical value $v_{\mathrm{c}}$ with $k=0, S_{-}{ }^{I}$ becomes stable with respect to arbitrary perturbations and any initially non-uniform distribution of species quickly evolves to the homogeneous integrated state after a short transient period.

The parameter region where the state of chaotic integration exists can be estimated from Fig. 5.5, where we have plotted the time needed for the disappearance of initially 

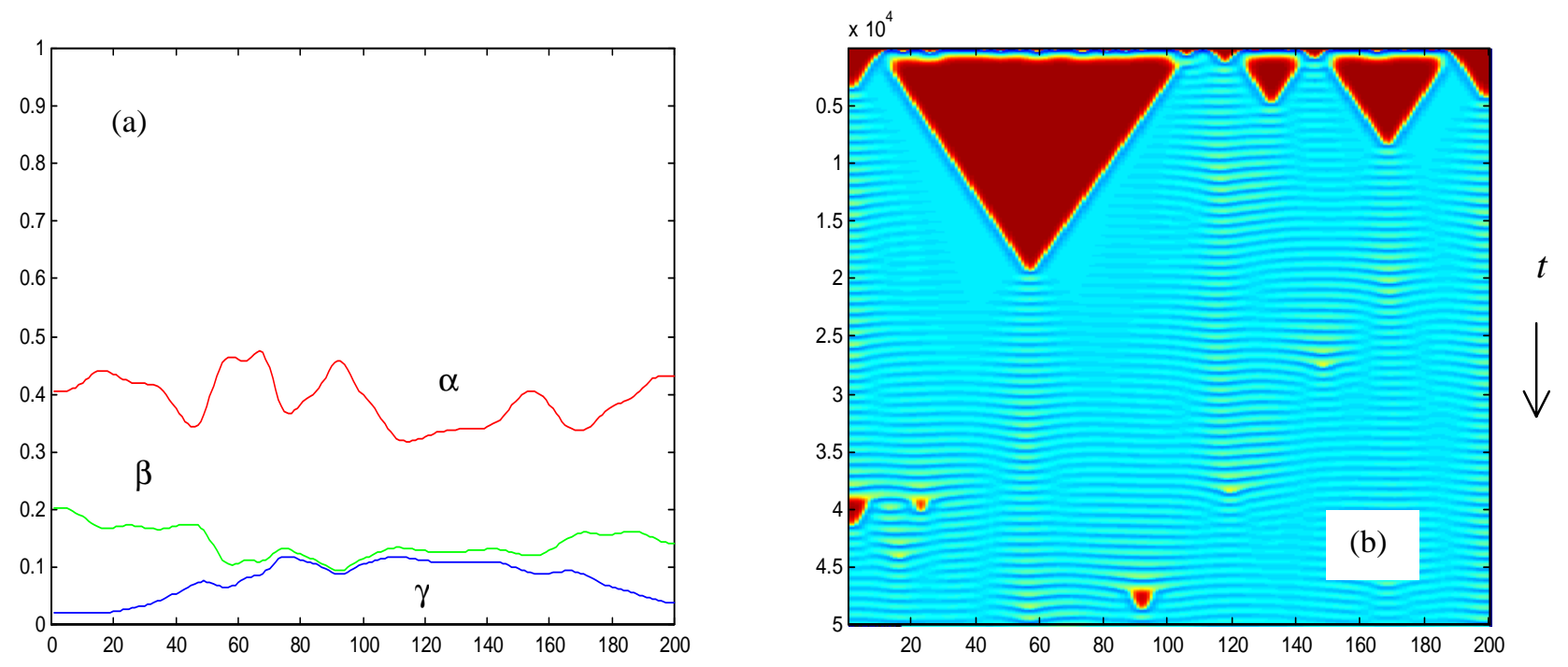

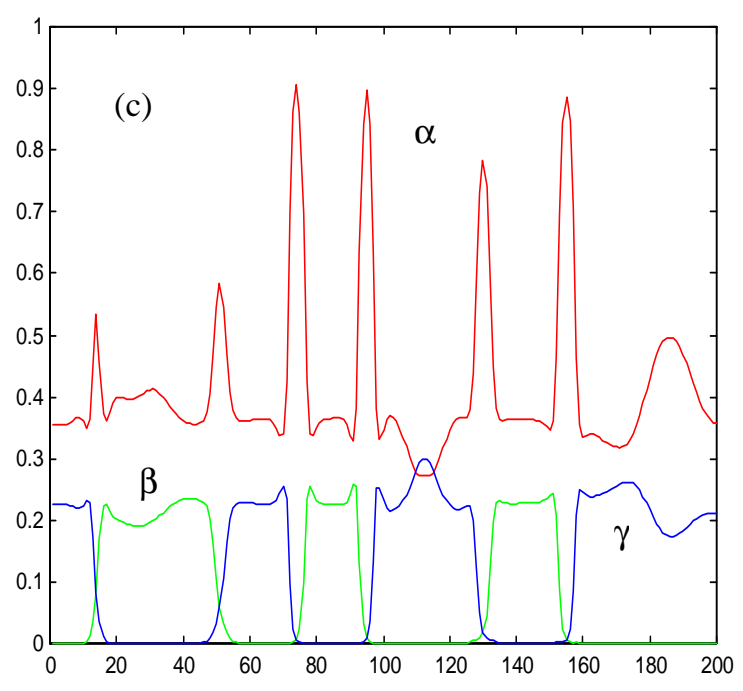

L

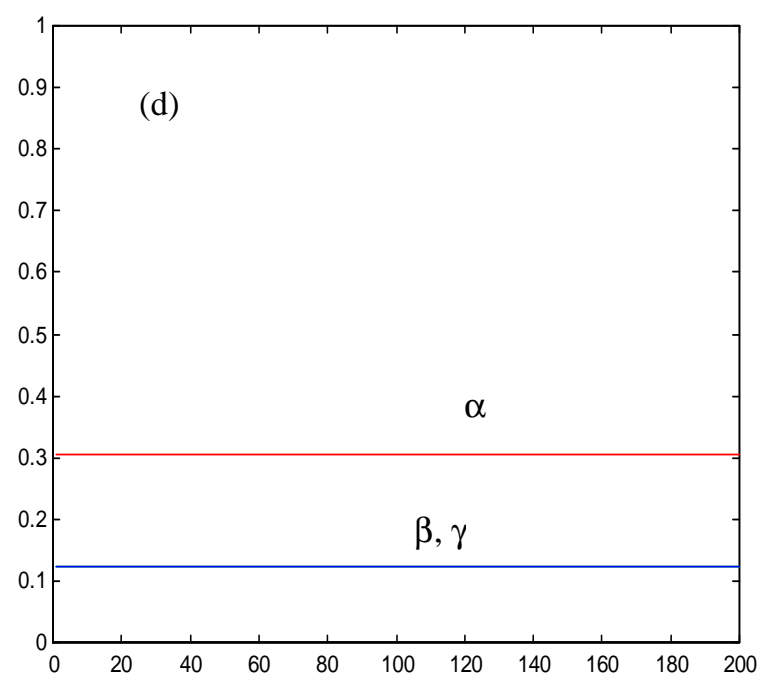

L

Figure 5.4. One-dimensional simulations of Eq. (5.3) with no-flux boundary conditions. Here $\phi=2.8, \mu=33.9, L=200, \delta L=0.25, t=50000$, and $\delta t=0.01$. (a) shows the final profiles of the concentrations at $v=34.1 \approx v_{\mathrm{c}}$. (b) shows the spatiotemporal evolution of the system, which is represented by the concentration of $\alpha$ (high concentration has darker color). (c) shows the phenomenon of segregation when $v=30.5<v_{c}$. (d) shows homogeneous integration when $v=40.5>v_{c}$. 


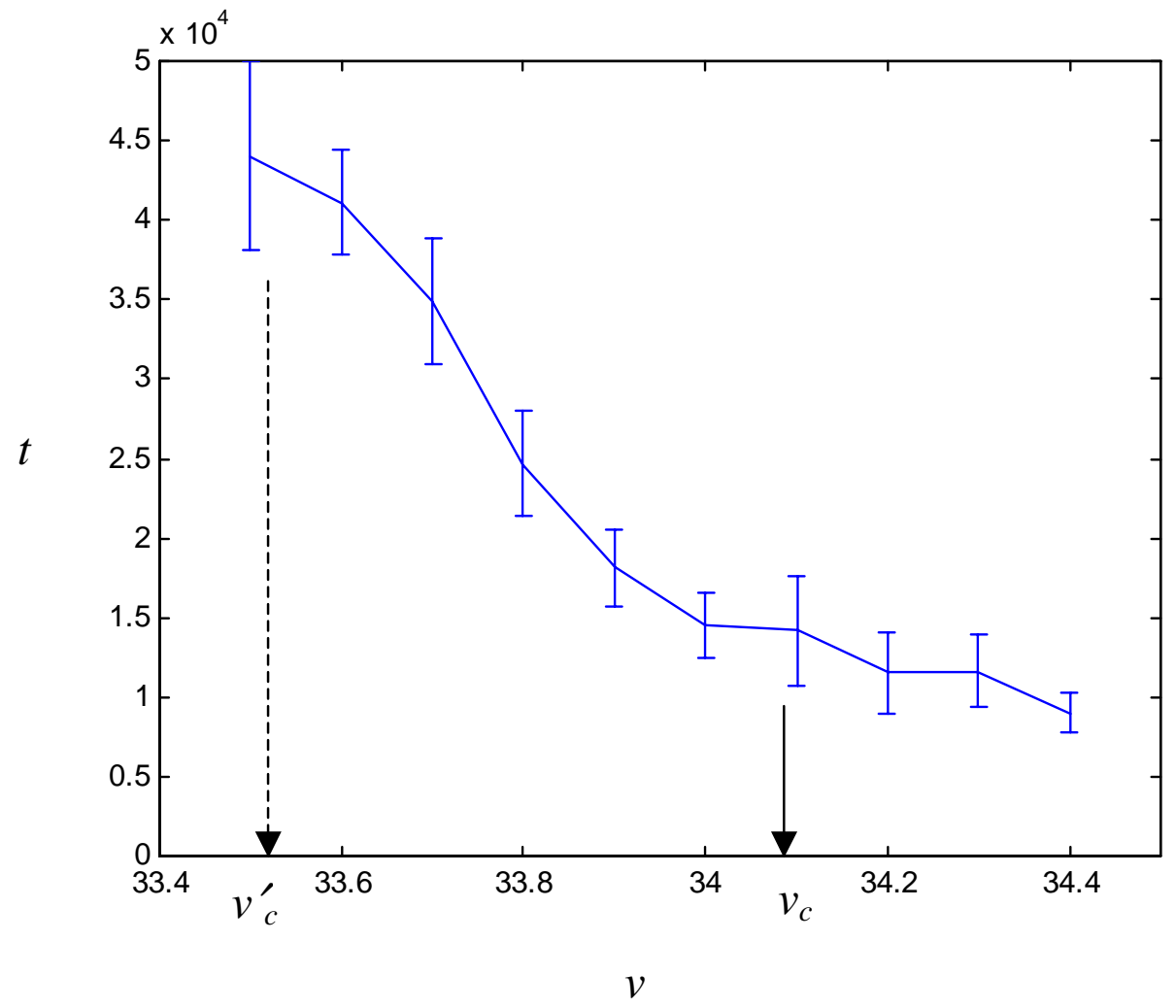

Figure 5.5. Measured average disappearing time of segregation w.r.t. parameter $v$ in the vicinity of the critical value $v_{\mathrm{c}}$. The disappearing time is based on measuring the time when the peak value of $\alpha$ falls below a level that is $50 \%$ above its stead state. 
formed domain walls versus the parameter $v$. The domain walls begin to disappear for $v_{\mathrm{c}}{ }^{\prime}$ $<v<v_{\mathrm{c}}$ and, as $v_{\mathrm{c}}$ is approached, the time needed for this transient decreases. Note that for $v>v_{\mathrm{c}}$, the system relaxes to stationary integration instead of chaotic integration. The gradual decrease of the transient time upon increasing $v$ can be rationalized from the fact that as $v$ increases more and more spatial modes are excited (cf. Eq. 5.11).

\subsubsection{Two-dimensional Simulation}

In the two-dimensional simulations, we set the system size to be $100 \times 100$ and finegrained the system into a square array of lattices with the size $200 \times 200$. The system was integrated for 20000 time steps with $\delta=0.02$, using the five-point Laplacian approximation and the explicit Euler method. Initially, the lattice points are initialized with uniform $\alpha=1$ and randomly distributed $\beta$ and $\gamma\left(\beta_{t=0}=1.0\right.$ and $\gamma_{t=0}=1.0$ at seeded lattice point; otherwise $\beta_{t=0}=0$ and $\gamma_{t=0}=0$ ). In addition, the initial distribution of $\beta$ and $\gamma$ was chosen in such a way that the sites where $\beta \neq 0$ and $\gamma \neq 0$ do not overlap, in order to monitor the disappearance of the boundaries between $\beta$ and $\gamma$. We chose no-flux boundary conditions and $\phi=2.8, \mu=33.92$, and $v=v_{\mathrm{B}}=v_{\mathrm{C}}=34.2$.

Fig. 5.6 shows the distribution of $\alpha$ at times $t=2000$ and $t=20000$. At $t=2000$, the system is still in the transient regime. Hence, we still observe the domain boundaries. Fig. 5.7 shows the initial and final patterns of $\beta$ and $\gamma$ at $t=0$ and $t=20000$, respectively. The 2D results are consistent with the 1D behavior: Although species $B$ and $C$ are initially segregated, they tend to evolve to an asymptotic state where they coexist throughout the spatial domain, i.e., the populations are integrated. For this choice of the parameters, the

final patterns are spatiotemporally chaotic. Thus, the curvature-induced drift of domain boundaries, as discussed in the study of the phenomenon of segregation, only plays a role in the transient process. 

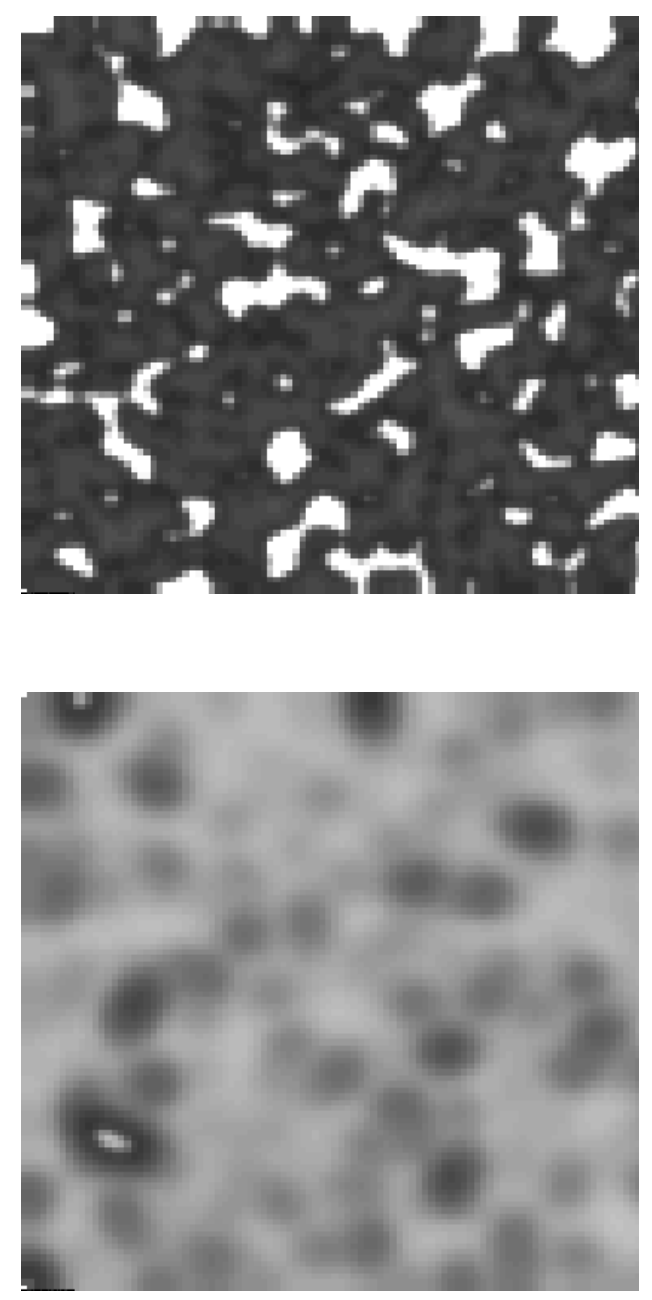

Figure 5.6. The upper panel shows a snapshot of the distribution of resource $\mathrm{A}$ at $\mathrm{t}=2000$, while the system is still relaxing to the final distribution. The lower panel shows the corresponding snapshot at $\mathrm{t}=20000 . \phi=2.8, \mu=$ $33.92, v=v_{\mathrm{B}}=v_{\mathrm{C}}=34.1$. 

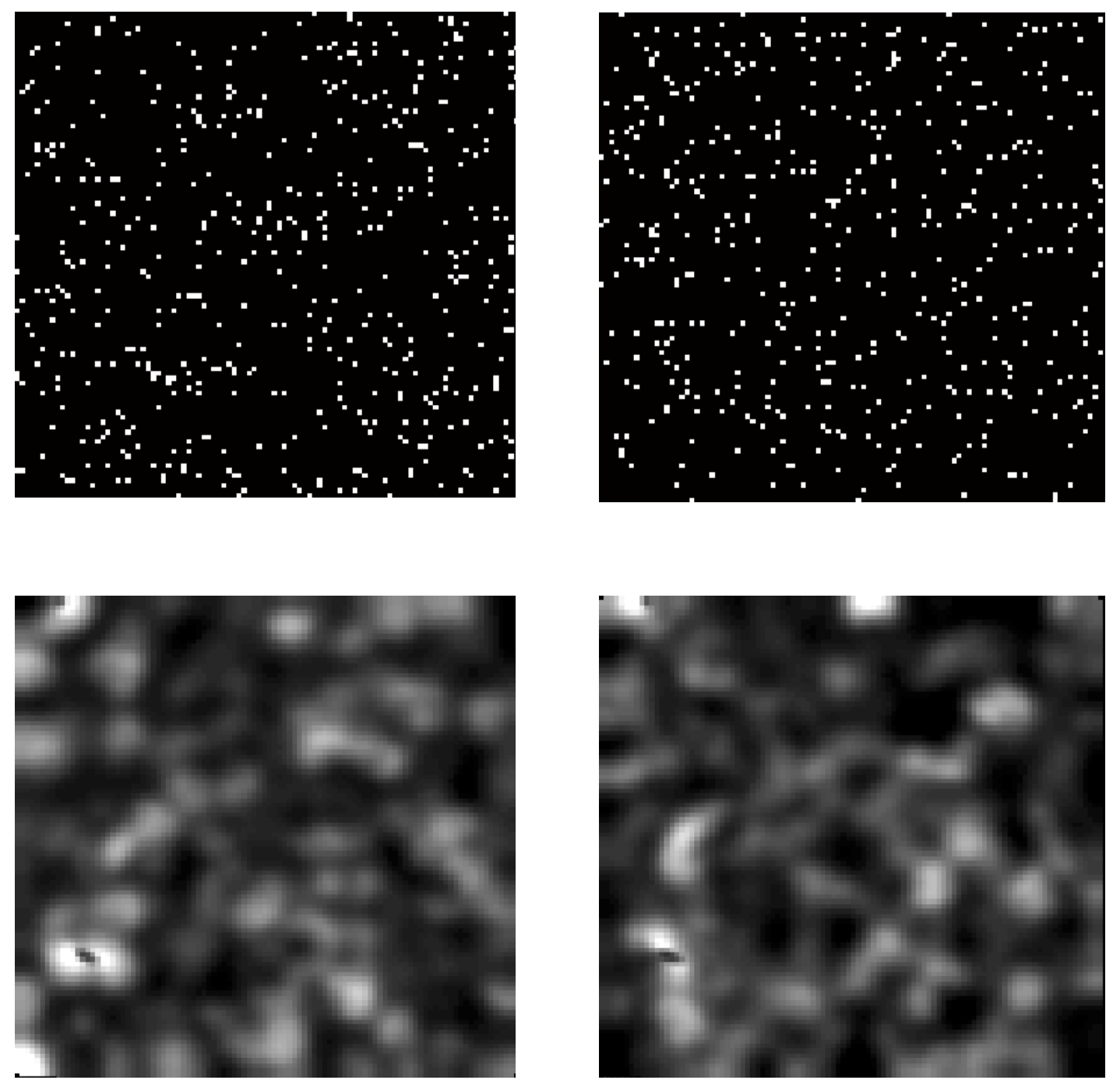

Figure 5.7. Upper two panels show the initial distribution of the species $B$ (left) and $C$ (right) for the case shown in Fig. 5.6. Lower panels show the corresponding patterns after 20000 integration steps. 
For other values of the parameters, we also found similar phenomena as in the onedimensional simulations. For example, when $\phi=2.8, \mu=33.92$, and $v=v_{\mathrm{B}}=v_{\mathrm{C}}=30.2$, the system exhibits segregation in a similar way as in Chapter 4 . When $\phi=2.8, \mu=$ 33.92 , and $v=v_{\mathrm{B}}=v_{\mathrm{C}}=38.2$, the system relaxes to the homogenous steady state, which corresponds to uniform integration.

\subsubsection{Discussion of the General Case}

If we take into account of different diffusivies, reaction rates, and decay rates as well as nonzero concentrations of $\beta$ and $\gamma$ in the reservoirs, i.e., $\beta_{0} \neq 0$ and $\gamma_{0} \neq 0$, the dimension of the entire parametric space would be as large as 10. It has not been possible in our study to explore the detailed behavior in the parametric space; however, we conclude from a limited series of calculations that close to the parametric conditions we studied above, the system exhibits similar dynamical behavior. For example, if we choose slightly different values for $v_{\mathrm{B}}$ and $v_{\mathrm{C}}$, we can find that in the homogenous steady state, the concentrations of $B$ and $C$ are different but close to each other (see Fig. 5.8). Moreover, we also observed chaotic integration when $v_{\mathrm{B}} \approx v_{\mathrm{C}} \sim v_{\mathrm{c}}$. However, if $v_{\mathrm{B}}>v_{\mathrm{C}}$ or vice versa, only one of the species can survive because of the asymmetry. On the other hand, if we also break the symmetry of the parameter $\mu$ and thus have $\mu_{\mathrm{B}} \neq \mu_{\mathrm{C}}$, the system can still exhibit the behavior observed in absence of broken symmetry, provided that the change is not too dramatic. The same holds as other parameters are varied. Thus, we can draw the conclusion that the phenomena discovered in this chapter are structurally stable. 

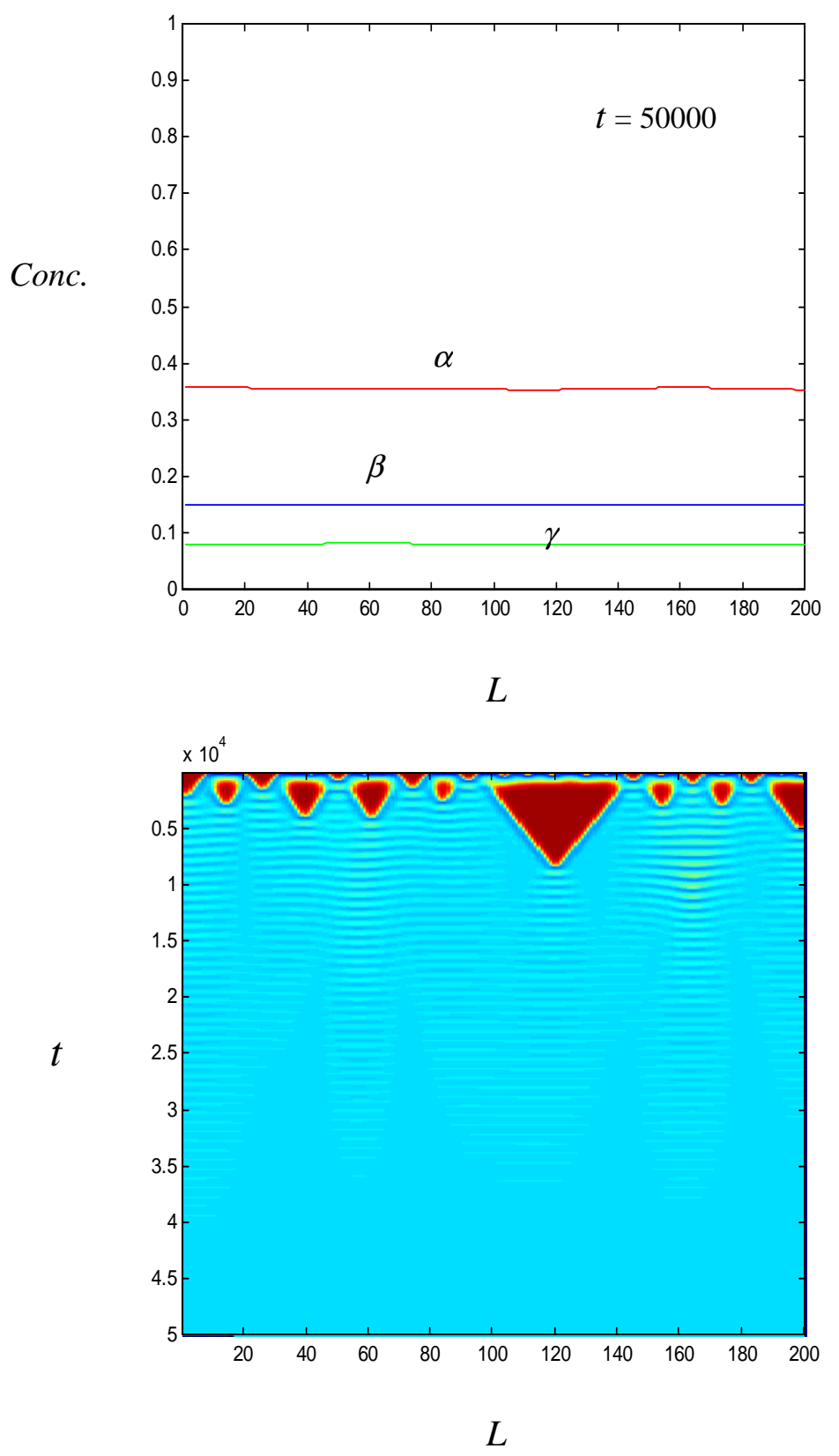

Figure 5.8. A one-dimensional demonstration of the case with symmetry broken $\left(v_{\mathrm{B}} \neq v_{\mathrm{C}}\right): \phi=2.8, \mu=33.92, v_{\mathrm{B}}=34.5$ and $v_{\mathrm{C}}=35$. (a) shows the concentration profiles at $\mathrm{t}=50000$. (b) shows the evolution of the concentration of $\alpha$. 


\subsection{Conclusion}

Dynamical systems with both competitive and cooperative processes are ubiquitous in nature. While in spatiotemporal systems competition may lead to the segregation of different species into separated spatial domains, cooperation can lead to the appearance of an integrated dynamics where multiple species coexist in the entire system. The LotkaVolterra model is a classical example of a system exhibiting segregation phenomena as a result of similar species competing for a common resource. However, models such as this do not include cooperative process among different species. Here, we have proposed a reaction-diffusion model incorporating both competitive and cooperative mechanisms. Analytical and numerical results show that the segregation of populations gives way to the integration of populations as the cooperation between the species becomes sufficiently strong. In addition, we note, according to this study, that chaotic integration only occurs close to certain critical conditions, although chaotic fluctuations of populations are generally observed in nature and have thus been the focus of many theoretical investigations. ${ }^{18}$ On one hand, this may imply some limitations of the model for explaining ecological systems and physical phenomena; on the other hand, it suggests that in population dynamics competition may be dominant as compared to cooperation. 


\subsection{Appendix:}

\subsubsection{Exact solutions of the eigenvalues}

In the system described in Chapter 4 , the third eigenvalue is always $-\phi$, and therefore the other eigenvalues are formed as solutions of a characteristic equation of second order. For the system described in this Chapter, such a situation does not exist, and thus we must find the solutions from a third-order characteristic equation.

Starting from the Jacobian matrix $J$ (Eq. (5.4) or (5.10)), we have the following form of characteristic equation,

$$
a \lambda^{3}+b \lambda^{2}+c \lambda+d=0 \quad(a \neq 0)
$$

where the coefficients can be expressed as

$$
\begin{aligned}
& a=-1, \\
& b=J_{11}+J_{22}+J_{33} \\
& c=-\left(J_{11} J_{22}+J_{22} J_{33}+J_{33} J_{11}\right)+\left(J_{12} J_{21}+J_{23} J_{32}+J_{31} J_{13}\right) \\
& d=J_{11}\left(J_{22} J_{33}-J_{23} J_{32}\right)-J_{12}\left(J_{21} J_{33}-J_{23} J_{31}\right)+J_{13}\left(J_{21} J_{32}-J_{22} J_{31}\right)
\end{aligned}
$$

and $J_{\mathrm{ij}}(\mathrm{i}, \mathrm{j}=1,2,3)$ are the corresponding matrix elements in Eq. (5.4) or (5.10). Divide Eq. (A1) by $a$ and let

$$
\lambda=\lambda^{\prime}-\frac{b}{3 a}
$$

Eq. (A1) then transforms into Cardan's equation:

$$
\lambda^{\prime 3}+p \lambda^{\prime}+q=0
$$


Its three roots can be written as

$$
\begin{aligned}
& \lambda^{\prime}{ }_{1}=\sqrt[3]{-\frac{q}{2}+\sqrt{\left(\frac{q}{2}\right)^{2}+\left(\frac{p}{3}\right)^{3}}}+\sqrt[3]{-\frac{q}{2}-\sqrt{\left(\frac{q}{2}\right)^{2}+\left(\frac{p}{3}\right)^{3}}} \\
& \lambda^{\prime}{ }_{2}=\omega \sqrt[3]{-\frac{q}{2}+\sqrt{\left(\frac{q}{2}\right)^{2}+\left(\frac{p}{3}\right)^{3}}}+\omega^{2} \sqrt[3]{-\frac{q}{2}-\sqrt{\left(\frac{q}{2}\right)^{2}+\left(\frac{p}{3}\right)^{3}}} \\
& \lambda^{\prime}{ }_{3}=\omega^{2} \sqrt[3]{-\frac{q}{2}+\sqrt{\left(\frac{q}{2}\right)^{2}+\left(\frac{p}{3}\right)^{3}}}+\omega \sqrt[3]{-\frac{q}{2}-\sqrt{\left(\frac{q}{2}\right)^{2}+\left(\frac{p}{3}\right)^{3}}}
\end{aligned}
$$

where $\omega=(-1+i \sqrt{ } 3) / 2, i^{2}=-1$. Accordingly, we obtain $\lambda_{I}=\lambda_{i}-\mathrm{b} /(3 a), i=1,2,3$. 


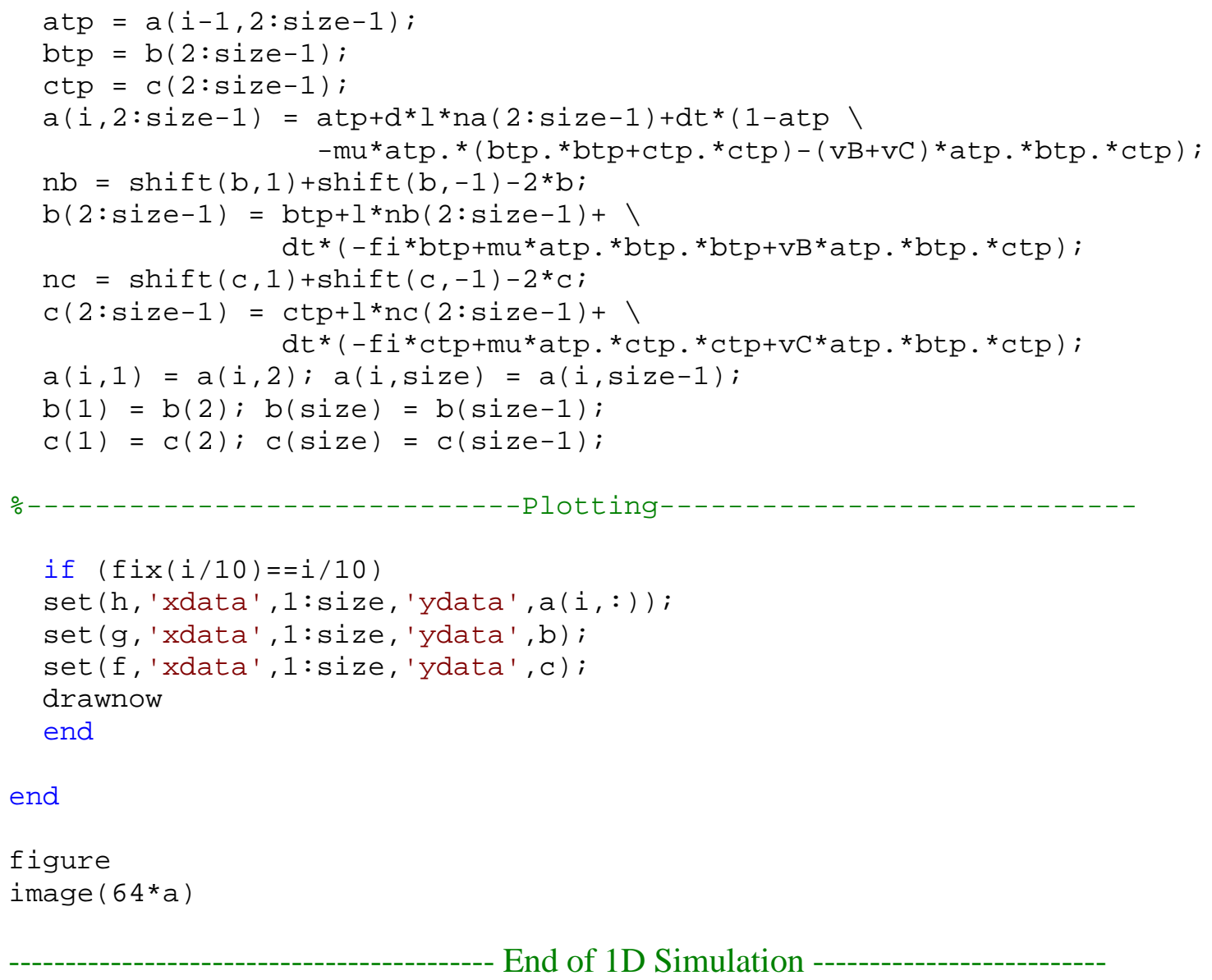

2. Two-dimensional Simulation

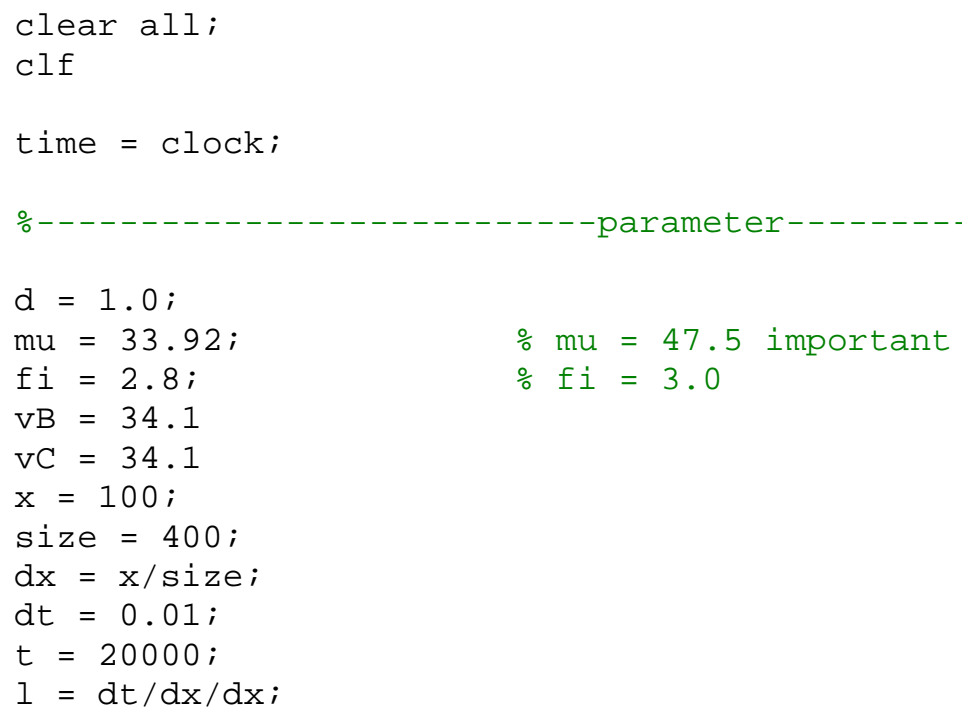




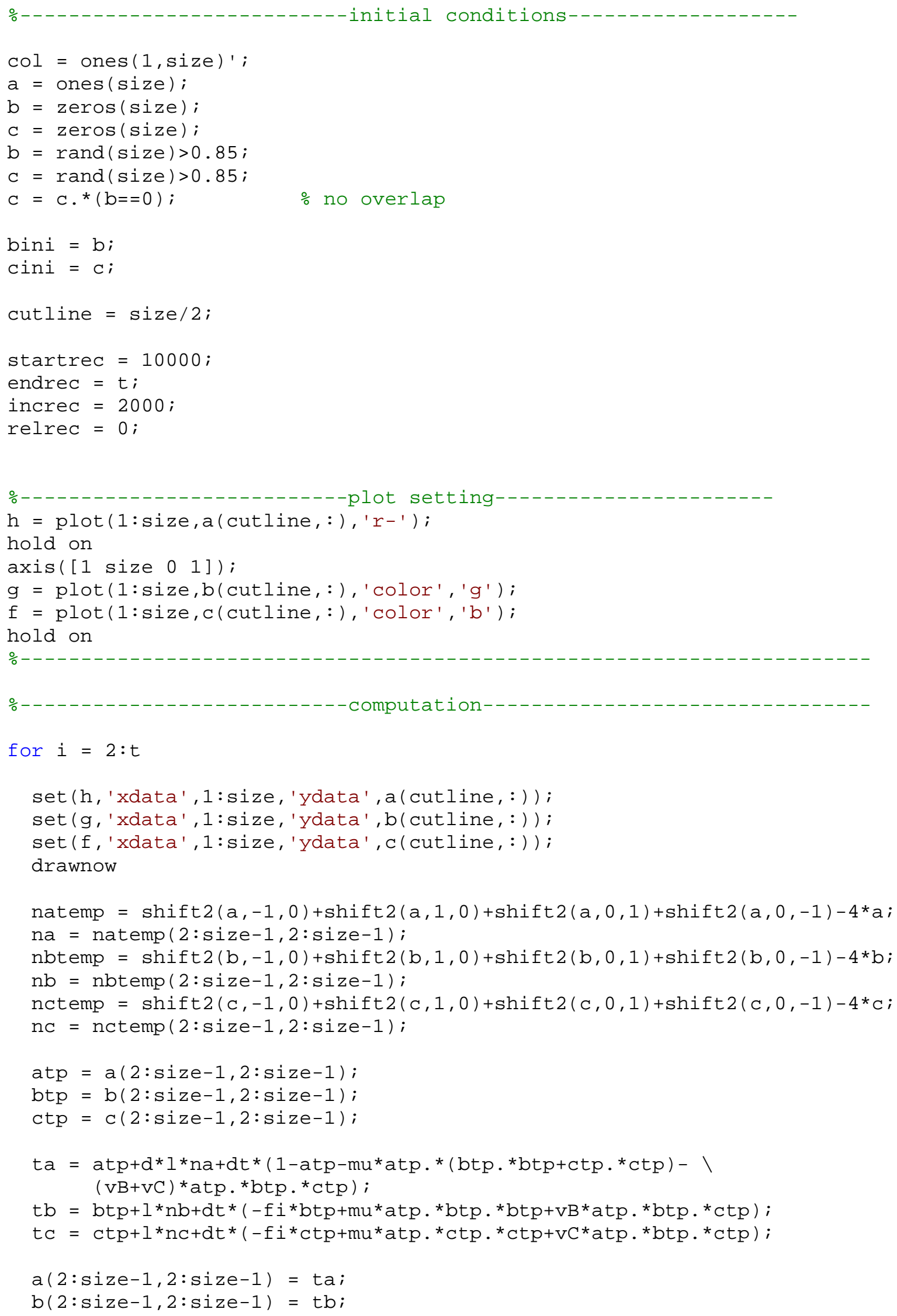




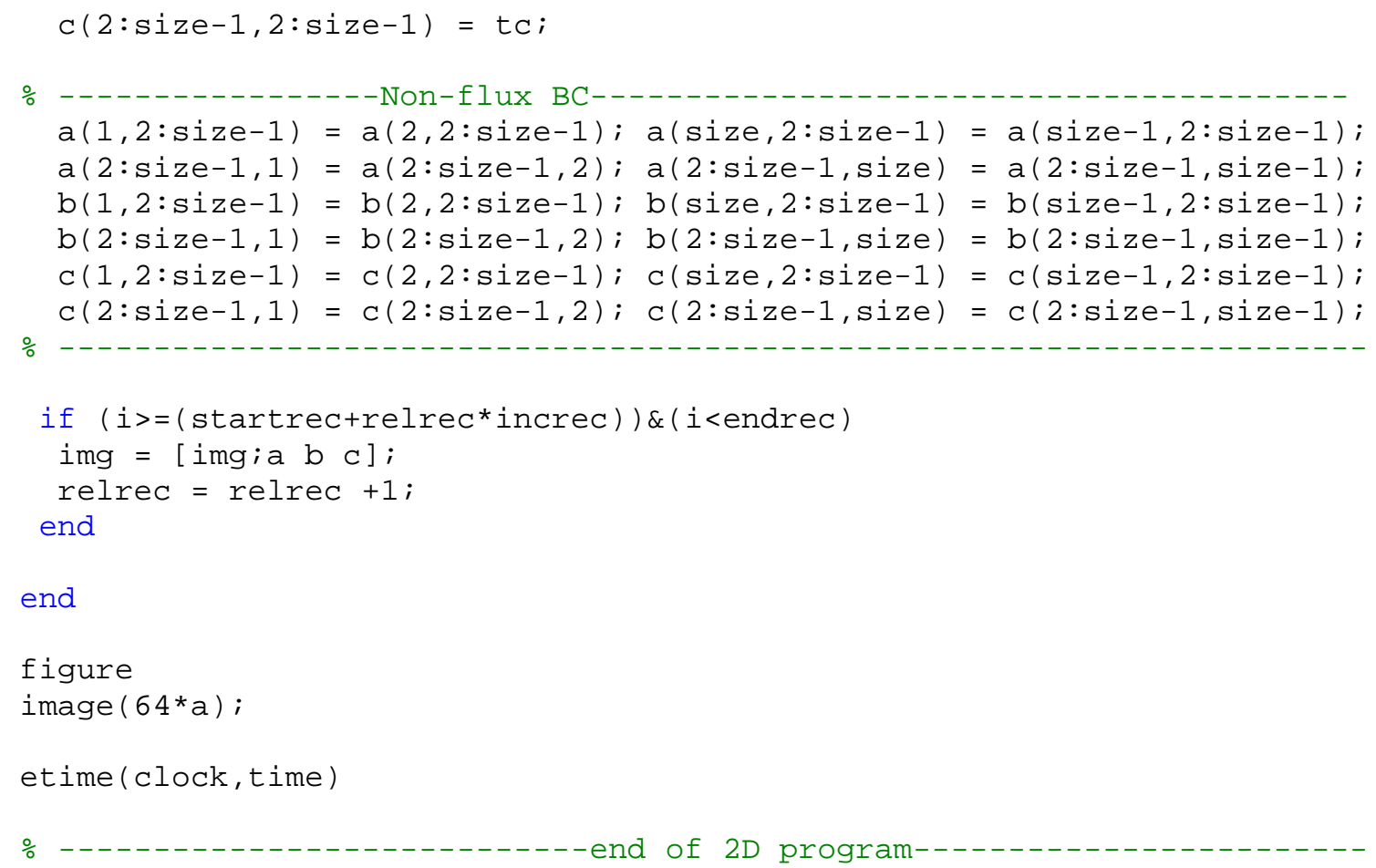




\subsection{References}

1. R. Wackerbauer, H. Sun, and K. Showalter, Phys. Rev. Lett 84, 5018 (2000).

2. G. Nicolis and I. Prigogine, Self-Organization in Non-Equilibrium Systems (Wiley, New York, 1977).

3. S. K. Scott, Chemical Chaos (Clarendon Press, Oxford, 1991).

4. E. Scholl, Nonequilibrium Phase Transitions in Semiconductors: Self-Organization Induced by Generation and Recombination Processes (Spring-Verlag, Berlin, Heidelberg, 1987).

5. H. Alfven, Cosmic Plasma (D. Reidel Publishing, New York, 1981).

6. H. Haken, Advanced Synergetics (Springer, Berlin, Heidelberg, 1983) and references therein.

7. M. C. Cross and P. C. Hohenberg, Pattern Formation Outside of Equilibrium, Rev. Mod. Phys. 65, No. 3 (1993).

8. M. P. Shaw, H. L. Grubin, E. Scholl, The Physics of Instabilities in Solid State Electron Devices, (Academic, New York 1989).

9. J. Casas-Vasquez and G. Lebon, Stability of Thermodynamic Systems, (Springer, Berlin, Heidelberg, 1982).

10. G. A. Held, C. Jeffries, E. E. Haller, Phys. Lett. 52, 1037 (1984).

11. M. Bulmer, Theoretical Evolutionary Ecology, (Sinauer Associates, Sunderland, Massachusetts, 1994).

12. J. Hofbauer and K. Sigmund, Evolutionary Games and Population Dynamics (Cambridge University Press, Oxford, 1998).

13. C. J. Avers, Process \& Pattern in Evolution (Oxford University Press, Oxford, 1989). 
14. P. H. Lindert, International Economics Ninth Edition (Irwin Press, Boston, 1991).

15. P. Gray and S. K. Scott, Chem. Engin. Sci. 39, 1087 (1984).

16. V. Petrov, S. K. Scott, and K. Showalter, Philos. Trans. R. Soc. London A 443, 631 (1994).

17. J. H. Merkin, V. Petrov, S. K. Scott, and K. Showalter, Phys. Rev. Lett. 76, 546 (1996).

18. R. M. May, Nature 261, 459 (1976). 